\title{
Dietary Patterns and Sarcopenia: A Systematic Review
}

\section{Dietary Guidelines Advisory Committee: Dietary Patterns Subcommittee \\ Published date: July 152020}

Nutrition Evidence Systematic Review Center for Nutrition Policy and Promotion

Food and Nutrition Service

U.S. Department of Agriculture

Braddock Metro Center II

1320 Braddock Place

Alexandria, Virginia 22314 
This systematic review was conducted by the 2020 Dietary Guidelines Advisory Committee in collaboration with the Nutrition Evidence Systematic Review (NESR) team at the Center for Nutrition Policy and Promotion, Food and Nutrition Service, U.S. Department of Agriculture (USDA). All systematic reviews from the 2020 Advisory Committee Project are available on the NESR website: https://nesr.usda.gov/2020-dietaryguidelines-advisory-committee-systematic-reviews.

Conclusion statements drawn as part of this systematic review describe the state of science related to the specific question examined. Conclusion statements do not draw implications, and should not be interpreted as dietary guidance. This portfolio provides the complete documentation for this systematic review. A summary of this review is included in the 2020 Advisory Committee's Scientific Report available at www.DietaryGuidelines.gov.

The contents of this document may be used and reprinted without permission. Endorsements by NESR, the Center for Nutrition Policy and Promotion, the Food and Nutrition Service, or the USDA of derivative products developed from this work may not be stated or implied.

Suggested citation for this systematic review: 2020 Dietary Guidelines Advisory Committee and Nutrition Evidence Systematic Review Team. Dietary Patterns and Sarcopenia: A Systematic Review. 2020 Dietary Guidelines Advisory Committee Project. Alexandria, VA: U.S. Department of Agriculture, Food and Nutrition Service, Center for Nutrition Policy and Promotion, July 2020. Available at: https://nesr.usda.gov/2020dietary-guidelines-advisory-committee-systematic-reviews.

Related citation: Dietary Guidelines Advisory Committee. 2020. Scientific Report of the 2020 Dietary Guidelines Advisory Committee: Advisory Report to the Secretary of Agriculture and the Secretary of Health and Human Services. U.S. Department of Agriculture, Agricultural Research Service, Washington, DC.

In accordance with Federal civil rights law and USDA civil rights regulations and policies, the USDA, its Agencies, offices, and employees, and institutions participating in or administering USDA programs are prohibited from discriminating based on race, color, national origin, religion, sex, gender identity (including gender expression), sexual orientation, disability, age, marital status, family/parental status, income derived from a public assistance program, political beliefs, or reprisal or retaliation for prior civil rights activity, in any program or activity conducted or funded by USDA (not all bases apply to all programs). Remedies and complaint filing deadlines vary by program or incident.

Persons with disabilities who require alternative means of communication for program information (e.g., Braille, large print, audiotape, American Sign Language, etc.) should contact the responsible Agency or USDA's TARGET Center at (202) 720-2600 (voice and TTY) or contact USDA through the Federal Relay Service at (800) 877-8339. Additionally, program information may be made available in languages other than English.

To file a program discrimination complaint, complete the USDA Program Discrimination Complaint Form, AD3027, found online at How to File a Program Discrimination Complaint and at any USDA office or write a letter addressed to USDA and provide in the letter all of the information requested in the form. To request a copy of the complaint form, call (866) 632-9992. Submit your completed form or letter to USDA by: (1) mail: U.S. Department of Agriculture, Office of the Assistant Secretary for Civil Rights, 1400 Independence Avenue, SW, Washington, D.C. 20250-9410; (2) fax: (202) 690-7442; or (3) email: program.intake@usda.gov.

USDA is an equal opportunity provider, employer, and lender. 


\section{Dietary Patterns Subcommittee:}

- Carol Boushey, PhD, MPH, RD, University of Hawaii, Subcommittee Chair

- Jamy Ard, MD, Wake Forest School of Medicine

- Lydia Bazzano, MD, PhD, Tulane University and Ochsner Health System

- Steven Heymsfield, MD, Louisiana State University, Pennington Biomedical Research Center

- Elizabeth Mayer-Davis, PhD, RD, University of North Carolina at Chapel Hill

- Joan Sabaté, MD, DrPH, Loma Linda University

- Linda Snetselaar, PhD, RDN, University of lowa

- Linda Van Horn, PhD, RDN, LD, Northwestern University

- Barbara Schneeman, PhD, University of California, Davis, Chair of the 2020 Dietary Guidelines Advisory Committee

\section{Nutrition Evidence Systematic Review (NESR) Team:}

- Laural Kelly English, PhD, Analyst, Panum Groupi

- Marlana Bates, MPH, RD, Analyst, Panum Group ${ }^{\mathrm{i}}$

- Emily Callahan, MS, Analyst, Office of Nutrition Guidance and Analysis (ONGA), Center for Nutrition Policy and Promotion (CNPP), Food and Nutrition Service (FNS), U.S. Department of Agriculture (USDA)

- Sudha Venkatramanan, PhD, Analyst, Panum Groupi

- Gisela Butera, MLIS, MEd, Systematic Review Librarian, Panum Groupi

- Nancy Terry, MS, MLS, Biomedical Librarian, National Institutes of Health (NIH) Library, U.S. Department of Health and Human Services (HHS)

- Julie Obbagy, PhD, RD, Project lead, ONGA, CNPP, FNS, USDA

\section{Federal Liaisons:}

- Elizabeth Rahavi, RD, ONGA, CNPP, FNS, USDA

- Clarissa (Claire) Brown, MS, MPH, RD, ONGA, CNPP, FNS, USDA

\section{Project Leadership:}

- Eve Essery Stoody, PhD, Designated Federal Officer and Director, ONGA, CNPP, FNS, USDA

- Janet de Jesus, MS, RD, Nutrition Advisor, Office of Disease Prevention and Health Promotion, Office of the Assistant Secretary for Health, HHS

USDA and HHS implemented a process to identify topics and scientific questions to be examined by the 2020 Dietary Guidelines Advisory Committee. The Committee conducted its review of evidence in subcommittees for discussion by the full Committee during its public meetings. The role of the Committee members involved establishing all aspects of the protocol, which presented the plan for how they would examine the scientific evidence, including the inclusion and exclusion criteria; reviewing all studies that met the criteria they set; deliberating on the body of evidence

\footnotetext{
i Under contract with the Food and Nutrition Service, United States Department of Agriculture.
} 
for each question; and writing and grading the conclusion statements to be included in the scientific report the 2020 Committee submitted to USDA and HHS. The NESR team with assistance from Federal Liaisons and Project Leadership, supported the Committee by facilitating, executing, and documenting the work necessary to ensure the reviews were completed in accordance with NESR methodology. More information about the 2020 Dietary Guidelines Advisory Committee, including the process used to identify topics and questions, can be found at www.DietaryGuidelines.gov. More information about NESR can be found at NESR.usda.gov.

The Committee and NESR staff thank USDA's Agricultural Research Service for coordinating the peer review of this systematic review, and the Federal scientist peer reviewers for their time and expertise.

FUNDING SOURCE: United States Department of Agriculture, Food and Nutrition Service, Center for Nutrition Policy and Promotion, Alexandria, VA 


\section{TABLE OF CONTENTS}

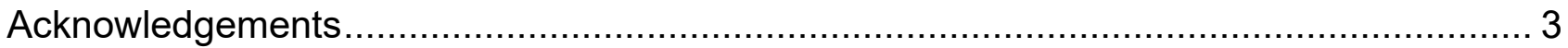

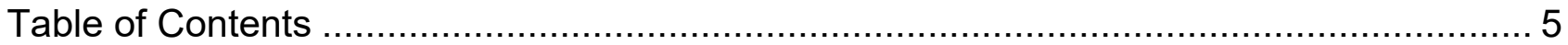

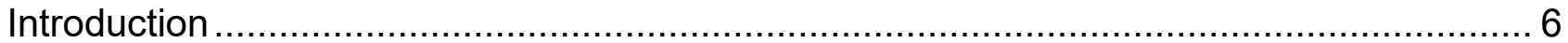

What is the relationship between dietary patterns consumed and sarcopenia?.................. 8

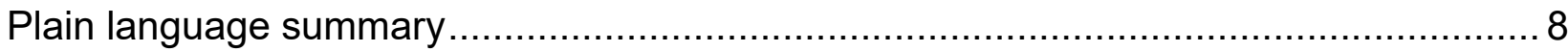

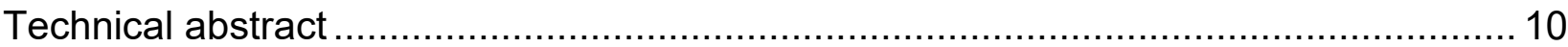

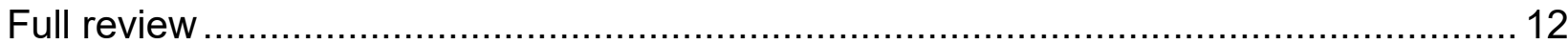

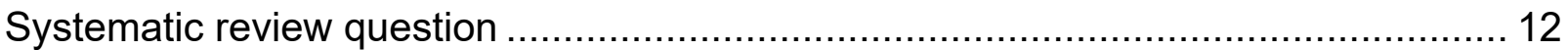

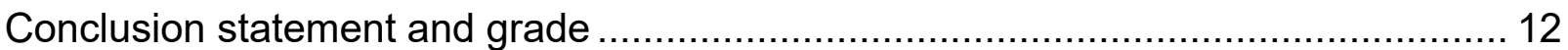

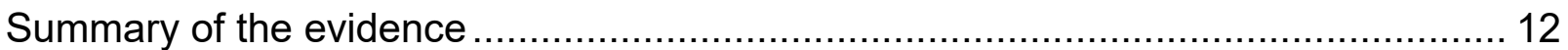

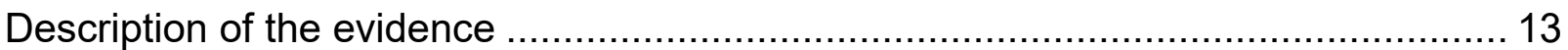

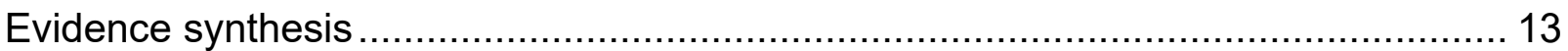

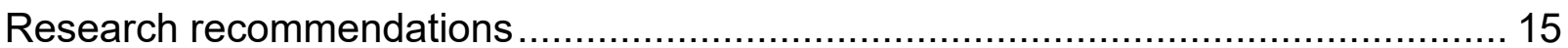

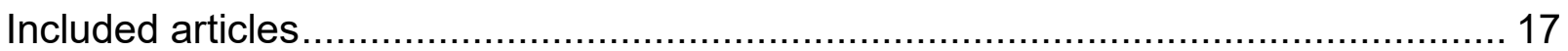

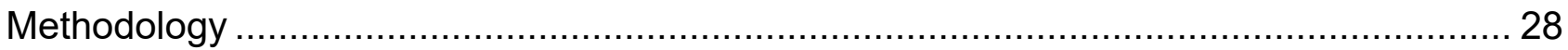

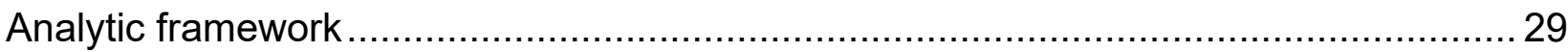

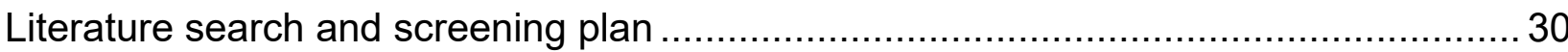

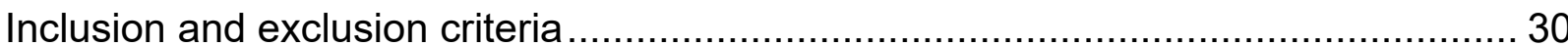

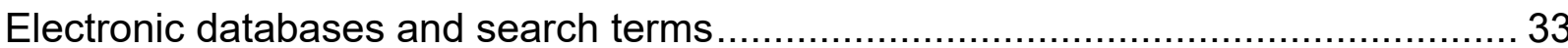

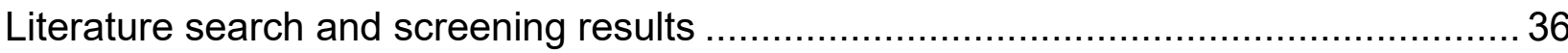

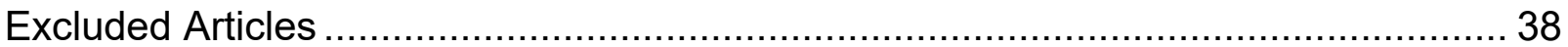

Table 1. Description of studies that examined the relationship between dietary patterns and diets based on macronutrient distribution and sarcopenia ......................................... 18 Table 2: Summary of the results from studies that examined the relationship between dietary patterns and diets based on macronutrient distribution and sarcopenia ................24 Table 3. Risk of bias for observational studies examining dietary patterns and diets based

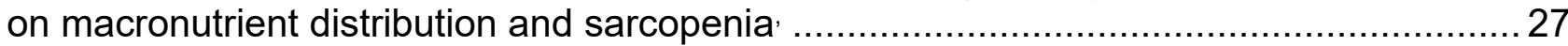

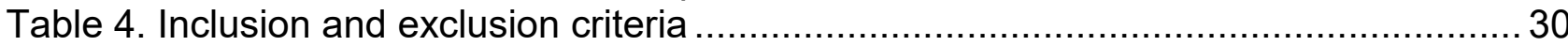

Table 5. Articles excluded after full text screening with rationale for exclusion...................38

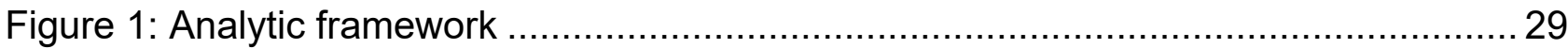

Figure 2: Flow chart of literature search and screening results................................... 37 
This document describes a systematic review conducted to answer the following question: What is the relationship between dietary patterns consumed and sarcopenia? This systematic review was conducted by the 2020 Dietary Guidelines Advisory Committee, supported by USDA's Nutrition Evidence Systematic Review (NESR).

More information about the 2020 Dietary Guidelines Advisory Committee is available at the following website: www.DietaryGuidelines.gov.

NESR specializes in conducting food- and nutrition-related systematic reviews using a rigorous, protocol-driven methodology. More information about NESR is available at the following website: NESR.usda.gov.

NESR's systematic review methodology involves developing a protocol, searching for and selecting studies, extracting data from and assessing the risk of bias of each included study, synthesizing the evidence, developing conclusion statements, grading the evidence underlying the conclusion statements, and recommending future research. A detailed description of the systematic reviews conducted for the 2020 Dietary Guidelines Advisory Committee, including information about methodology, used in conducting systematic reviews for the 2020 Dietary Guidelines Advisory Committee is available on the NESR website: https://nesr.usda.gov/2020-dietary-guidelines-advisory-committee-systematicreviews. In addition, starting on page 28 , this document describes the final protocol as it was applied in the systematic review. A description of and rationale for modifications made to the protocol are described in the 2020 Dietary Guidelines Advisory Committee Report, Part D: Chapter 8. Dietary Patterns. 


\section{List of abbreviations}

\begin{tabular}{ll}
\hline Abbreviation & Full name \\
\hline AMDR & Acceptable macronutrient distribution range \\
\hline DQI-I & Diet quality index - international \\
\hline EWGSOP & European Working Group on Sarcopenia in Older Adults \\
\hline HHS & United States Department of Health and Human Services \\
\hline mHDI & Modified Healthy Diet Indicator \\
\hline MDS & Mediterranean diet score \\
\hline NESR & Nutrition Evidence Systematic Review \\
\hline ONGA & Office of Nutrition Guidance and Analysis \\
\hline PCS & Prospective cohort study \\
\hline SES & Socioeconomic status \\
\hline USDA & United States Department of Agriculture
\end{tabular}




\section{WHAT IS THE RELATIONSHIP BETWEEN DIETARY PATTERNS CONSUMED AND SARCOPENIA?}

\section{PLAIN LANGUAGE SUMMARY}

What is the question?

- The question is: What is the relationship between dietary patterns consumed and sarcopenia?

What is the answer to the question?

Dietary patterns

- Insufficient evidence is available to determine the relationship between dietary patterns and sarcopenia in older adults.

\section{Diets based on macronutrient distribution}

- Insufficient evidence was available to determine the relationship between diets based on macronutrient distribution and sarcopenia.

\section{Why was this question asked?}

- This important public health question was identified by the U.S. Departments of Agriculture (USDA) and Health and Human Services (HHS) to be examined by the 2020 Dietary Guidelines Advisory Committee.

\section{How was this question answered?}

- The 2020 Dietary Guidelines Advisory Committee, Dietary Patterns Subcommittee conducted a systematic review to answer this question with support from the Nutrition Evidence Systematic Review (NESR) team.

- Dietary patterns were defined as the quantities, proportions, variety, or combination of different foods, drinks, and nutrients (when available) in diets, and the frequency with which they are habitually consumed.

- Diets based on macronutrient distribution were examined when at least one macronutrient proportion was outside of the acceptable macronutrient distribution range (AMDR) for carbohydrate, fat, and/or protein.

What is the population of interest?

- For the intervention/exposure, adolescents through older adults

- For the outcome, adults and older adults

What evidence was found?

- This review identified 4 articles that met inclusion criteria.

- Four articles examined dietary patterns and sarcopenia.

- Two articles also examined diets based on macronutrient distribution and sarcopenia.

- Studies differed in dietary pattern methods, macronutrient distributions, and assessment of sarcopenia.

- Results from the included studies were inconsistent. 
- Several limitations in the study design and conduct of included articles were identified. This includes a lack of accounting for potential confounders or possible changes in dietary intake over time.

- The 2020 Committee could not draw conclusions due to limitations in and lack of evidence.

How up-to-date is this systematic review?

- This review searched for studies from January, 2000 and October, 2019. 


\section{TECHNICAL ABSTRACT}

\section{Background}

- This important public health question was identified by the U.S. Departments of Agriculture (USDA) and Health and Human Services (HHS) to be examined by the 2020 Dietary Guidelines Advisory Committee.

- The 2020 Dietary Guidelines Advisory Committee, Dietary Patterns Subcommittee conducted a systematic review to answer this question with support from the Nutrition Evidence Systematic Review (NESR) team.

- The goal of this systematic review was to examine the following question: What is the relationship between dietary patterns consumed and sarcopenia?

\section{Conclusion statements and grades}

\section{Dietary patterns}

- Insufficient evidence is available to determine the relationship between dietary patterns and sarcopenia in older adults. (Grade: Grade not assignable)

\section{Diets based on macronutrient distribution}

- Insufficient evidence was available to determine the relationship between diets based on macronutrient distribution and sarcopenia. (Grade: Grade not assignable)

\section{Methods}

- Two literature searches were conducted using 3 databases (PubMed, Cochrane, Embase) to identify articles that evaluated the intervention or exposure of dietary patterns consumed and the outcomes of sarcopenia. A manual search was conducted to identify articles that may not have been included in the electronic databases searched. Articles were screened by two NESR analysts independently for inclusion based on pre-determined criteria.

- Data extraction and risk of bias assessment were conducted for each included study, and both were checked for accuracy. The Committee qualitatively synthesized the body of evidence to inform development of a conclusion statement(s), and graded the strength of evidence using pre-established criteria for risk of bias, consistency, directness, precision, and generalizability.

\section{Summary of the evidence}

- This systematic review includes 4 prospective cohort studies that examined the relationship between dietary patterns and sarcopenia, 2 of which also examined diets based on macronutrient distribution, that met inclusion criteria, and were published between January 2000 and October 2019.

- Two of the studies reported macronutrient distributions in which the percent of energy from fat was higher than the AMDR.

- Dietary patterns were defined as the quantities, proportions, variety, or combination of different foods, drinks, and nutrients (when available) in diets, and the frequency with which they are habitually consumed.

- Diets based on macronutrient distribution were examined when at least one macronutrient proportion was outside of the acceptable macronutrient distribution range (AMDR) for carbohydrate, fat, and/or protein. When describing and categorizing studies included in this review, the Committee 
did not label the diets examined as "low" or "high," because no universally accepted, standard definition is currently available, for example, for "lowcarbohydrate" or "high-fat" diets. Instead, the Committee focused on whether, and the extent to which, the proportions of the macronutrients were below or above the AMDR

- The studies were inconsistent, both in terms of which dietary patterns or macronutrient distributions that were examined, how dietary intake was assessed, assessment of sarcopenia, and results reported regarding the association between dietary patterns and risk of sarcopenia. In addition, the studies had relatively small sample sizes with few cases of sarcopenia.

- The body of evidence had several risks of bias, including lack of adjustment for all potential confounders, and assessment of diet only once at baseline, and lack of accounting for possible changes in dietary intake that may have occurred over follow-up.

- The studies were direct and generalizable, in that that the intervention, comparators, and outcomes of interest in the included studies are directly related to the systematic review question, and are applicable to the U.S. population. However, study participants may have been healthier than the average older adult. 


\section{FULL REVIEW}

\section{Systematic review question}

What is the relationship between dietary patterns consumed and sarcopenia?

\section{Conclusion statements and grades}

\section{Dietary patterns}

Insufficient evidence is available to determine the relationship between dietary patterns and sarcopenia in older adults. (Grade: Grade not assignable)

\section{Diets based on macronutrient distribution}

Insufficient evidence was available to determine the relationship between diets based on macronutrient distribution and sarcopenia. (Grade: Grade not assignable)

\section{Summary of the evidence}

- This systematic review includes 4 prospective cohort studies that examined the relationship between dietary patterns and sarcopenia, 2 of which also examined diets based on macronutrient distribution, that met inclusion criteria and were published between January 2000 and October 2019.1-4

- Two of the studies reported macronutrient distributions in which the percent of energy from fat was higher than the AMDR. ${ }^{2,3}$

- Dietary patterns were defined as the quantities, proportions, variety, or combination of different foods, drinks, and nutrients (when available) in diets, and the frequency with which they are habitually consumed.

- Diets based on macronutrient distribution were examined when at least one macronutrient proportion was outside of the acceptable macronutrient distribution range (AMDR) for carbohydrate, fat, and/or protein. When describing and categorizing studies included in this review, the Committee did not label the diets examined as "low" or "high," because no universally accepted, standard definition is currently available, for example, for "low-carbohydrate" or "high-fat" diets. Instead, the Committee focused on whether, and the extent to which, the proportions of the macronutrients were below or above the AMDR.

- The studies were inconsistent, both in terms of which dietary patterns or macronutrient distributions that were examined, how dietary intake was assessed, assessment of sarcopenia, and results reported regarding the association between dietary patterns and risk of sarcopenia. In addition, the studies had relatively small sample sizes with few cases of sarcopenia.

- The body of evidence had several risks of bias, including lack of adjustment for all potential confounders, and assessment of diet only once at baseline, and lack of accounting for possible changes in dietary intake that may have occurred over follow-up.

- The studies were direct and generalizable, in that that the intervention, comparators, and outcomes of interest in the included studies are directly related to the systematic review question, and are applicable to the U.S. population. However, study participants may have been healthier than the average older adult. 


\section{Description of the evidence}

This systematic review includes 4 prospective cohort studies that examine the relationship between dietary patterns and sarcopenia, 2 of which also examined diets based on macronutrient distribution that met inclusion criteria, and were published between January 2000 and October 2019 (Table 1). ${ }^{1-4}$

\section{Population/participant characteristics}

The studies included in this systematic review were conducted in China, ${ }^{1}$ Finland, ${ }^{3}$ Sweden, ${ }^{4}$ and the United Kingdom. ${ }^{2}$ Sample size ranged from 254 to 2948 participants. Two studies included both men and women, ${ }^{1,2}$ one study included only women, ${ }^{3}$ and one study included only men. ${ }^{4}$ Mean age of participants at baseline ranged from approximately 68 years to $>85$ years.

\section{Intervention/exposure}

Included studies examined dietary patterns using a variety of methods. One study identified dietary patterns using factor analysis, ${ }^{1}$ one study identified dietary patterns using cluster analysis, ${ }^{2}$ and 3 studies examined adherence to dietary patterns using 6 different indices or scores. ${ }^{1,3,4}$ Two studies ${ }^{2,3}$ examined dietary patterns in which the proportion of energy from fat was above the AMDR in at least one of the exposure groups compared (i.e., each cluster ${ }^{2}$ or third quartile ${ }^{3}$ ).

Dietary intake was assessed using a variety of validated methods, including a foodfrequency questionnaire, ${ }^{1}$ 24-hour dietary recall using a multiple pass method, ${ }^{2}$ and 3 day $^{3}$ or 7 -day ${ }^{4}$ food records. In all studies, diet was assessed once, at baseline.

\section{Outcome assessment}

All included studies examined risk of developing sarcopenia, with follow-up ranging from 3-4 years ${ }^{1-3}$ to 16 years. ${ }^{4}$

One study defined sarcopenia using the Asian Working Group for Sarcopenia algorithm, ${ }^{1}$ and 3 studies defined sarcopenia using the European Working Group on Sarcopenia in Older People (EWGSOP) criteria. ${ }^{2-4}$ In all of the included studies, sarcopenia was defined as low muscle mass with low muscle strength (based on handgrip strength) and/or low muscle performance (based on walking speed). However, Isanejad et $\mathrm{al}^{3}$ included participants considered to be pre-sarcopenic (low muscle mass only) or sarcopenic in analyses. ${ }^{3}$

\section{Evidence synthesis}

Results reported in the studies included in this systematic review were mixed (Table 2). Chan et al ${ }^{1}$ analyzed data from a prospective cohort study from Hong Kong that included both men and women ( $51 \%$ female), aged $\sim 72$ years. ${ }^{1}$ Five dietary patterns were examined, including 2 dietary patterns based on an index/score (Dietary Quality Index-International score (DQI-I), Mediterranean Diet Score (MDS)), and 3 identified via factor analysis ("Vegetables-Fruits", "Snacks-Drinks-Milk Products", and "MeatFish"). None of the dietary patterns examined were significantly associated with risk of sarcopenia after 4 years of follow-up. ${ }^{1}$

Granic et $\mathrm{al}^{2}$ analyzed data from a prospective cohort study from the United Kingdom 
that included both men and women (62\% female), all over 85 years of age. ${ }^{2}$ Three dietary patterns were examined, that were identified via cluster analyses ('Low Red Meat', 'Traditional British', 'Low Butter'). All 3 patterns had a mean percent energy from fat higher than the AMDR. Adherence to a 'Traditional British' compared to a 'Low Butter' dietary pattern at $>85$ years was associated with higher risk of prevalent sarcopenia at 3 year follow-up. However, it was not significantly associated with incident sarcopenia. Adherence to a 'Low Red Meat' compared to a 'Low Butter' dietary pattern was not significantly associated with risk of prevalent or incident sarcopenia. In addition, results were similar when stratified by protein intake $>1$ or $<1 \mathrm{~g} / \mathrm{kg}$ adjusted body weight/day. ${ }^{2}$

Isanejad et $\mathrm{al}^{3}$ analyzed data from a prospective cohort study from Finland that included only women, aged $\sim 68$ years. ${ }^{3}$ Two dietary patterns were examined that were based on an index/score (Baltic Sea Diet Score (BSD) and MDS. For the BSD score, one quartile had percent energy from fat higher than the AMDR. Higher BSD score (categorical) at 68 years was significantly associated with reduced risk of sarcopenia after 3 year follow-up. However, associations were not significant when BSD was analyzed continuously. MDS score at 68 years was not significantly associated with risk of sarcopenia after 3 year follow-up when analyzed either continuously or categorically. In this study, pre-sarcopenic and sarcopenic women were combined, and analyzed together. ${ }^{3}$

Karlsson et al ${ }^{4}$ analyzed data from a prospective cohort study from Sweden that included only men, aged $\sim 71$ years. ${ }^{4}$ Two dietary patterns were examined that were based on an index/score (Modified Healthy Diet Indicator (mHDI), modified Mediterranean Diet Score (mMDS)). Higher mHDI score (medium vs. low) at 71 years was significantly associated with lower risk of sarcopenia at 87 years. However, high vs. low score and analysis of scores continuously were not significantly associated with risk of sarcopenia. Higher mMDS score (continuous) at 71 years was associated with lower risk of sarcopenia at 87 years. However, when mMDS scores were analyzed categorically (low vs. medium or high), there were no significant associations with risk of sarcopenia. ${ }^{4}$

\section{Assessment of the evidence ${ }^{\text {ii }}$}

This systematic review included only 4 studies that met inclusion criteria, and all were prospective cohort studies. It was determined that this body of evidence was insufficient to draw conclusions about the relationship between dietary patterns and risk of sarcopenia, or diets based on macronutrient distribution and risk of sarcopenia. As outlined and described in detail below, the body of evidence was assessed for the following elements used when grading the strength of evidence.

- Risk of bias: The included articles had a number of potential risks of bias, or limitations, that make interpretation of results difficult (Table 1; Table 3). While

\footnotetext{
ii A detailed description of the methodology used for grading the strength of the evidence is available on the NESR website: https://nesr.usda.gov/2020-dietary-guidelines-advisory-committee-systematicreviews and in Part $\mathrm{C}$ of the following reference: Dietary Guidelines Advisory Committee. 2020. Scientific Report of the 2020 Dietary Guidelines Advisory Committee: Advisory Report to the Secretary of Agriculture and the Secretary of Health and Human Services. U.S. Department of Agriculture, Agricultural Research Service, Washington, DC.
} 
studies adjusted for a number of potential confounders, they did not adjust for all key confounders, such as race/ethnicity or physical disability. These studies examined dietary patterns only once at baseline, and did not account for possible changes in dietary intake that may have occurred over follow-up. In addition, the studies enrolled older individuals, and did not account for dietary patterns consumed earlier in life. None of the studies accounted for missing data, either due to lost to follow-up or criteria used when selecting subjects into the analyses. Attrition due to mortality was not reported by the included articles. The studies are also prone to selection bias. Though subjects enrolled in the study were intended to be representative of the general population in the country or region where the study was conducted, they likely represent healthier individuals (i.e., those able to walk, take public transportation, or with interest in preventing future falls or fracture). Finally, none of the studies had preregistered statistical plans, and therefore, there is potential for selective outcome reporting.

- Consistency: The studies were inconsistent, both in terms of which dietary patterns were examined, how dietary intake was assessed, assessment of sarcopenia, and results reported regarding the association between dietary patterns and risk of sarcopenia.

- Directness: The populations, intervention, comparators, and outcomes of interest in the included studies are directly related to the systematic review question.

- Precision: The studies had small analytic sample sizes with relatively few sarcopenia cases. The width of confidence intervals indicates imprecision within the body of evidence.

- Generalizability: The interventions and/or exposures, comparators, and outcomes examined in the body of evidence are applicable to the U.S. population. However, study participants may have been healthier than the average older adult (i.e., those able to walk, take public transportation, or with interest in preventing future falls or fracture)

\section{Research recommendations}

In order to better assess the relationship between dietary patterns and sarcopenia, future research may:

1. Assess information regarding diet at more than one time-point, preferably during the course of follow-up, to facilitated determining the relationship between dietary. patterns over time and sarcopenia.

2. Examine the relationship between dietary patterns earlier in life and risk of sarcopenia.

3. Provide sufficient information, definitions or standards, and repeated measures on the quantification, i.e., types and amounts of foods/food groups such as fruits and vegetables, and beverages such as alcohol, consumed when examining the relationship between dietary patterns and/or diets based on macronutrient distribution and sarcopenia. 
4. Include diverse populations with varying race/ethnicity, age, sex/gender, socioeconomic background, and chronic disease status, while ensuring to report the racial/ethnic background of participants studied. 


\section{Included articles}

1. Chan R, Leung J, Woo J. A prospective cohort study to examine the association between dietary patterns and sarcopenia in Chinese community-dwelling older people in Hong Kong. $J \mathrm{Am}$ Med Dir Assoc. 2016;17(4):336-342. doi:10.1016/j.jamda.2015.12.004.

2. Granic A, Mendonca N, Sayer AA, et al. Effects of dietary patterns and low protein intake on sarcopenia risk in the very old: the Newcastle 85+ study. Clin Nutr. 2020;39(1):166-173.

doi:10.1016/j.clnu.2019.01.009.

3. Isanejad M, Sirola J, Mursu J, et al. Association of the Baltic Sea and Mediterranean diets with indices of sarcopenia in elderly women, OSPTRE-FPS study. Eur J Nutr. 2018;57(4):1435-1448. doi:10.1007/s00394-017-1422-2.

4. Karlsson M, Becker W, Michaelsson K, Cederholm T, Sjogren P. Associations between dietary patterns at age 71 and the prevalence of sarcopenia 16 years later. Clin Nutr. 2020;39(4):10771084. doi:10.1016/j.clnu.2019.04.009. 
Table 1. Description of studies that examined the relationship between dietary patterns and diets based on macronutrient distribution and sarcopeniaiii

\section{Study and \\ Participant \\ Characteristics \\ Chan, $2016^{1}$ \\ PCS (Mr. Os and Ms \\ Os), \\ Hong Kong, China}

Analytic N: 2948

(Attrition: 26\%)

Sex: $51 \%$ female

Age: 72.4 y [5.0]

Anthropometry: mean

BMI $23.6 \mathrm{~kg} / \mathrm{m}^{2}$ [3.1]

Physical activity:

Physical activity scale

for the elderly (PASE):

91.2 [39.8]

SES: Education:

$71.5 \%$ primary or

below; Marital status:

$\sim 70 \%$ married or

cohabited

Alcohol: 12\%

currently drink alcohol
Intervention/Exposure and Outcomes

Dietary patterns:

- 2 dietary patterns were examined using an index/score:

o Dietary Quality Index-International score (DQI-I) iv

- Positive components: Vegetables, fruit, grain, fiber, protein, iron, calcium vitamin $\mathrm{C}$, overall food group variety, within food group variety

- Moderation components: Total fat, SFA, cholesterol, sodiu, empty calorie foods, macronutrient ratio, fatty acid ratio

- Mediterranean Diet Score (MDS) ${ }^{\vee}$

- Positive components: Vegetables, legumes, fruit and nuts, cereals, fish, MUFA:SFA ratio

- Negative components: Red and processed meat, dairy products

- Moderation components: Alcohol

- 3 dietary patterns identified via factor analysis:

○ "Vegetables-Fruits": Higher in tomatoes,

dark green and leafy vegetables,

cruciferous vegetables, starchy

vegetables, other vegetables, soy,

fruits, legumes, mushroom and fungi,

and lower in fats and oils

Results

Confounding, Study Limitations, and Summary of Findings

\section{Significant: N/A}

\section{Non-Significant}

In men $(n=1449)$, dietary patterns at

$72 \mathrm{y}$ and sarcopenia at $4 \mathrm{y} \mathrm{f} / \mathrm{u}$ :

- DQI-I: OR: 1.00, 95\% Cl: 0.98, 1.02, $\mathrm{p}=0.670, \mathrm{NS}$

- MDS: OR: 0.98, 95\% Cl: 0.86, 1.10, $\mathrm{P}=0.678$, NS

- Vegetables-fruits: OR: 1.07, 95\% Cl: $0.88,1.29, p=0.522, \mathrm{NS}$

- Snacks-drinks-milk products: OR: 0.95, Cl: 0.78, 1.16, $p=0.611$, NS

- Meat-fish: OR: 1.00, 95\% Cl: 0.84, 1.20, $p=1.000$, NS

In women ( $n=1499)$, dietary patterns at $72 \mathrm{y}$ and sarcopenia at $4 \mathrm{y} \mathrm{f} / \mathrm{u}$ :

- DQI-I: OR: 0.99, CI: 0.97, 1.02, $p=0.611, N S$

- MDS: OR: 0.96, Cl: 0.83, 1.11, $p=0.602$, NS

- Vegetables-fruits: OR: 1.15, Cl: $0.94,1.40, p=0.190, \mathrm{NS}$

- Snacks-drinks-milk products: OR: 1.20, Cl: $0.94,1.53, p=0.148, N S$ Meat-fish: OR: $1.10, \mathrm{Cl}: 0.87,1.40$ $\mathrm{p}=0.415$, NS

iii Abbreviations: AMDR, Acceptable macronutrient distribution range; C, dietary carbohydrate; CI, confidence interval; CRP, C-reactive protein; DQI-I, Diet quality index -

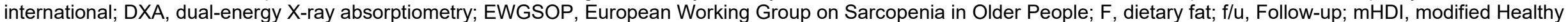

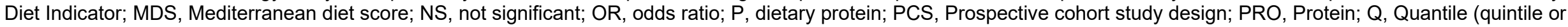
quartile as appropriate); RR, Relative risk; SES, Socioeconomic status; y, years

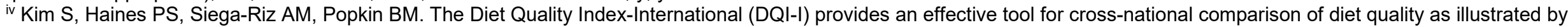
China and the United States. J Nutr 2003;133: 3476e3484.

$\checkmark$ Trichopoulou A, Costacou T, Bamia C, Trichopoulos D. Adherence to a Mediterranean diet and survival in a Greek population. N Engl J Med 2003;348: 2599e2608. 


Study and
Participant
Characteristic

PCS (The Newcastle

85+ Study),

United Kingdom

Analytic N: 373

(Attrition: 64\%)

Age: $\geq 85 y$

Sex: $62 \%$ female

SES: $51 \%$ manual occupation; $64 \%$ 0-9y
○ "Snacks-Drinks-Milk Products": Higher in condiments, coffee, fast food, nuts, French fries and potato chips, milk and milk products, whole grains, sweets and desserts, beverage

o "Meat-Fish": Higher in dim sum, red and processed meats, poultry, fish and seafood, wine, and lower in refined grains

Dietary assessment methods: Validated FFQ at baseline, age $\sim 72 \mathrm{y}$

\section{Outcome assessment methods:}

Sarcopenia was defined according to the Asian

Working Group for Sarcopenia algorithm:

- Low muscle mass: Appendicular skeletal muscle mass (ASM) index, ASM/height ${ }^{2}<7.0$ $\mathrm{kg} / \mathrm{m}^{2}$ for men, $<5.4 \mathrm{~kg} / \mathrm{m}^{2}$ for women

- Low muscle strength: Grip strength $<26 \mathrm{~kg}$ for men, <18g for women

- Low physical performance: Gait speed <0.8 $\mathrm{m} / \mathrm{s}$ for men and women

\section{Dietary patterns:}

Adherence to 3 dietary patterns identified via cluster analysis:

- 'Low Red Meat': lower in legumes, sweets and desserts, and higher in fish/seafood

- 'Traditional British': higher in butter, red meats and meat dishes, gravy, potatoes and potato dishes, vegetables, and sweets/desserts

- 'Low Butter': Higher in unsaturated fat spreads and oils

Diet based on macronutrient distribution, \% energy from carbohydrate $(C)$, fat $(F)$, protein $(P)$ :

- 'Low Red Meat': 47\% C, 37\% F, 16\% P

- 'Traditional British': 46\% C, 39\% F, 15\% P

Summary: Adherence to the DQI-I, MDS, 'vegetables-fruits', 'snacks-drinks-milk products', or 'meat-fish' dietary patterns at $72 y$ was not significantly associated with risk of sarcopenia after $4 y \mathrm{f} / \mathrm{u}$.

\section{Significant:}

'Traditional British' vs. 'Low Butter' pattern at age $\geq 85 y$ and prevalent sarcopenia at $3 \mathrm{y}$ f/u: OR: $2.42,95 \%$ Cl: 1.15, 5.09, $p=0.02$.

When stratified by protein intake $>1$ or $<1 \mathrm{~g} / \mathrm{kg}$ aBW/d, results were only significant in those with protein intake $\geq 1.0 \mathrm{~g} / \mathrm{kg}$ BW/d: OR: $3.62,95 \% \mathrm{Cl}$ : $1.33,9.88, p=0.01$

\section{Non-significant}

'Low Red Meat' vs. 'Low Butter" pattern at age $\geq 85 y$ and prevalent
Key confounders accounted for: Sex; SES; Anthropometry; Physical activity; Dietary protein intake

Other: Food energy; Depression; Cognitive status; Total number of chronic diseases; Total number of medications

\section{Limitations}

- Did not account for the following key confounders: Age; Race/ethnicity; Physical disability

- Exposure occurred prior to start of f/u

- Only assessed dietary intake once at baseline; did not account for possible changes in dietary intake over $\mathrm{f} / \mathrm{u}$ 
Results

Confounding, Study Limitations, and

Summary of Findings

\begin{tabular}{|c|c|}
\hline $\begin{array}{l}\text { Study and } \\
\text { Participant } \\
\text { Characteristics }\end{array}$ & Intervention/Exposure and Outcomes \\
\hline $\begin{array}{l}\text { education; } 69 \% \\
\text { married }\end{array}$ & - 'Low Butter': 48\% C, 35\% F, 17\% P \\
\hline $\begin{array}{l}\text { Anthropometry: } 40 \% \\
\text { overweight/obese }\end{array}$ & $\begin{array}{l}\text { Dietary assessment methods: } 24-h \text { multiple } \\
\text { pass dietary recall at baseline, } \geq 85 y\end{array}$ \\
\hline $\begin{array}{l}\text { Dietary protein intake: } \\
63.75 \mathrm{~g} / \mathrm{d}\end{array}$ & $\begin{array}{l}\text { Outcome assessment methods: Sarcopenia } \\
\text { was defined using the European Working Group } \\
\text { on Sarcopenia in Older Adults (EWGSOP) } \\
\text { criteria: }\end{array}$ \\
\hline $\begin{array}{l}\text { Physical activity: } 19 \% \\
\text { low, } 45 \% \text { moderate, } \\
36 \% \text { high }\end{array}$ & $\begin{array}{l}\text { - Low skeletal muscle index (<8.87 kg/m2 in } \\
\text { men, }<6.67 \mathrm{~kg} / \mathrm{m} 2 \text { in women; via } \\
\text { bioimpedance analysis); and } \\
\text { - Slow gait speed ( } \leq 0.8 \mathrm{~m} / \mathrm{s} \text {, via TUG test), } \\
\text { and/or } \\
\text { Weak grip strength (<16 kg in women, }<26 \\
\text { kg in men; via dynamometer while } \\
\text { standing). }\end{array}$ \\
\hline
\end{tabular}

sarcopenia at $3 y \mathrm{f} / \mathrm{u}, \mathrm{n}=356$ : OR:

$1.77,95 \% \mathrm{Cl}: 0.84,3.74, p=0.13$, NS

'Low Red Meat' vs. 'Low Butter" pattern at age $\geq 85 y$ and incident sarcopenia at $3 y \mathrm{f} / \mathrm{u}, \mathrm{n}=288$ : OR: $1.05,95 \% \mathrm{Cl}: 0.37,3.03, p=0.92$, NS.

'Traditional British' vs. 'Low Butter' pattern at age $\geq 85 y$ and incident sarcopenia at $3 y \mathrm{f} / \mathrm{u}, \mathrm{n}=288$ : OR: 1.67, 95\%Cl: $0.59,4.67, p=0.33$, NS

Results were similar when stratified by protein intake $>1$ or $<1 \mathrm{~g} / \mathrm{kg}$ aBW/d.
- Did not account for missing data

- No published protocol; potential for selective outcome reporting

\section{Funding Sources:}

European Horizon 2020 PROMISS Project; National Institute for Health Research

Newcastle Biomedical Research Centre Tyne Hospitals NHS Foundation Trust; Newcastle University; UK Medical

Research Council; Biotechnology and Biological Sciences Research Council; Dunhill Medical Trust; the Medical Council Research grant; NHS North of Tyne

Summary: Adherence to a 'Traditional British' compared to a 'Low Butter' dietary pattern at $>85 y$ was associated with higher risk of prevalent sarcopenia at $3 y \mathrm{f} / \mathrm{u}$. However, it was not significantly associated with incident sarcopenia.

Adherence to a 'Low Red Meat' compared to a 'Low Butter' dietary pattern was not significantly associated with risk of prevelant or incident sarcopenia. Key confounders accounted for: Sex; Age; SES; Anthropometry; Physical activity; Physical disability

BSD scores at $68 y$ and sarcopenia after $3 y \mathrm{f} / \mathrm{u}$ :

- Q1: OR: 1.00

PCS, Osteoporosis

Adherence to 2 dietary patterns was examined:

- Baltic Sea Diet Score (BSD) vi

- Q2: OR: $0.82,95 \% \mathrm{Cl}: 0.30,2.24$

Positive components: Total fruits (mostly apples, pears) and berries, vegetables (root vegetables, legumes, nuts,

Prevention Study mushrooms, and vegetable products, and excluding potatoes), fiber from cereal products, fish, low-fat milk, PUFA: SFA ratio
-Q3: OR: $0.76,95 \% \mathrm{Cl}: 0.27,2.13$

- Q4: OR: $0.33,95 \% \mathrm{Cl}: 0.13,0.79$

- $p=0.015$
Other: Energy intake, Smoking, Hormone Therapy, Osteoporosis, Rheumatoid Arthritis, Coronary Heart Disease, Depression, Diabetes
Finland

${ }^{v i}$ Kanerva N, Kaartinen NE, Schwab U, Lahti-Koski M, Männistö S (2014) The Baltic Sea Diet Score: a tool for assessing healthy eating in Nordic countries. PHN 17(8):1697-1705 


\section{Study and \\ Participant \\ Characteristics}

Analytic N: 554

(Attrition: 26\%)

Age: 67.8y [1.85]

Sex: $100 \%$ female

SES: Income of 883.3 euros/mo [311.1]

Anthropometry: BMI of $26.5 \mathrm{~kg} / \mathrm{m}^{2}$ [3.6]

Total energy intake:

6576.5 kJ/d [1541]

Dietary protein intake: $67.85 \mathrm{~g} / \mathrm{d}$ [18.0]

Physical activity: 47.5 $\mathrm{h} / \mathrm{mo}$ [46.4]

Physical

disability: falls in past $12 \mathrm{mo} 21.8 \%$
Intervention/Exposure and Outcomes

Results

Confounding, Study Limitations, and

Summary of Findings

- Did not account for the following key confounders: Race/ethnicity; Dietary protein intake

- Subjects volunteered for a study on falls/fracture prevention; exposure occurred prior to start of $\mathrm{f} / \mathrm{u}$

- Only assessed dietary intake once at baseline; did not account for possible changes in dietary intake over $\mathrm{f} / \mathrm{u}$

- Did not account for missing data

- Combined pre-sarcopenic and sarcopenic women

- No published protocol; likely selective outcome reporting

Diet based on macronutrient distribution:

Total energy intake, and macronutrients/alcoho in $\mathrm{g} / \mathrm{d}$ by quartiles of dietary patterns scores are after $3 y \mathrm{f} / \mathrm{u}$ :

- Q1: OR: 1.00

- Q2: OR: $0.87,95 \% \mathrm{Cl}: 0.33,2.25$

- Q3: OR: $1.20,95 \% \mathrm{Cl}: 0.44,3.23$

- Q4: OR: $0.75,95 \% \mathrm{Cl}: 0.28,2.05$ $p=0.751$

\section{BSD scores}

- Q1: $47.7 \%$ C, 34.9\% F, 15.9\% P

- Q2: $48.3 \%$ C, $32.1 \% \mathrm{~F}, 16.7 \% \mathrm{P}$

- Q3: $49.9 \%$ C, $41.1 \% \mathrm{~F}, 13.1 \% \mathrm{P}$

- Q4: $50.8 \%$ C, $27.1 \% \mathrm{~F}, 18.5 \% \mathrm{P}$

MDS scores

- Q1: $48.4 \%$ C, 31.7\% F, 17.5\% P

- Q2: $49.1 \%$ C, $30.8 \%$ F, $17.8 \%$ P

- Q3: $49.7 \%$ C, $31.1 \% \mathrm{~F}, 17.2 \% \mathrm{P}$

- Q4: $51.4 \%$ C, $29.9 \%$ F, $17.1 \%$ P

Dietary assessment methods: 3-d food record at baseline, age 68y

Outcome assessment methods: Sarcopenia was defined using EWGSOP criteria:

- Relative skeletal muscle index (RSMI) (lowest

\section{Funding Sources:}

The Finnish Cultural Foundation, Sigrid Juselius Foundation, Academy of Finland, Kuopio University Hospital, Päivikki and Sakari Sohlberg Foundation, Finnish Cultural Foundation, North Savo Regional Fund, Otto. A Malm Foundation, Yrjö Jahnsson Foundation, Juho Vainio Foundation

Summary: Higher BSD score (categorical) at $68 y$ was significantly associated with reduced risk of sarcopenia after $3 y \mathrm{f} / \mathrm{u}$. However, associations were not significant when BSD was analzyed continuously.

MDS score at 68y was not signicantly associated with risk of sarcopenia after $3 y$ $f / u$.

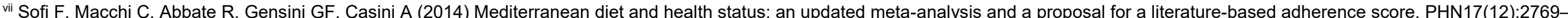

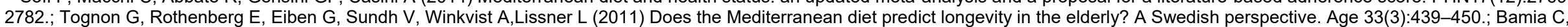
Lagiou P, Buckland G, Grioni S, Agnoli C, Taylor AJ et al (2013) Mediterranean diet and colorectal cancer risk: results from a European cohort. Eur J Epidemiol 28(4):317-328. 


\begin{tabular}{|c|c|c|c|}
\hline $\begin{array}{l}\text { Study and } \\
\text { Participant } \\
\text { Characteristics }\end{array}$ & Intervention/Exposure and Outcomes & Results & $\begin{array}{l}\text { Confounding, Study Limitations, and } \\
\text { Summary of Findings }\end{array}$ \\
\hline
\end{tabular}

quartile, via DXA), and,

- Handgrip strength (any quartile, via dynamometer of dominant hand taken while sitting), and/or

- Walking speed (any quartile, time of $10 \mathrm{~m}$ walk)

\section{Karlsson, $2019^{4}$ \\ PCS, Uppsala Longitudinal Study of Adult Men (ULSAM), Sweden}

Analytic N: 254 (Attrition: 78\%)

Age: $70.9 y,[0.6]$

Sex: $0 \%$ female

SES: $48 \% 6-7 y$ education, $48 \%$ high school education, $31 \%$ university educationow education

Anthropometry: BMI of $25.9 \mathrm{~kg} / \mathrm{m}^{2}$ [2.9]

Dietary protein intake: $42 \%<0.8 \mathrm{~g} / \mathrm{kg}, 34 \%$

$0.8-1.0 \mathrm{~g} / \mathrm{kg}, 25 \%>1.0$ $\mathrm{g} / \mathrm{kg}$

Physical activity: $68 \%$ regular

\section{Significant:}

Adherence to 2 dietary patterns was examined:

- Modified Healthy Diet Indicator (mHDI)

- Positive components: PUFA, protein, carbohydrates, fiber, fruit and vegetables, fish

- Negative components: Sucrose, SFA, cholesterol

- Modified Mediterranean Diet Score (mMDS)

o Positive components: PUFA:SFA ratio, vegetables and legumes, fruit and berries, cereals and potatoes, fish

- Negative components: Meat and meat products, milk and milk products

o Moderation component: Alcohol

Dietary assessment methods: $7-d$ weighed food record at baseline, age 71y

Outcome assessment methods: Sarcopenia was defined using EWGSOP criteria:

- Low muscle mass (skeletal muscle mass index $<7.26 \mathrm{~kg} / \mathrm{m}$; via DXA)

- Low handgrip strength (<30 kg; via dynamometer of dominant hand taken while sitting)

- Low muscle performance (gait speed $<0.8 \mathrm{~m} / \mathrm{s}$; via intermediate $6 \mathrm{~m}$ of a $10 \mathrm{~m}$ walk)
mHDI score (medium vs. low) at $71 \mathrm{y}$ and sarcopenia at $87 y$ : OR: 0.38 $95 \% \mathrm{Cl}: 0.15,0.97$

mMDS score (continuous) at $71 \mathrm{y}$ and sarcopenia at age $87 y$ : OR: 0.49 $95 \% \mathrm{Cl}: 0.31,0.80$

\section{Non-Significant:}

mHDI score (high vs. low) at $71 \mathrm{y}$ and sarcopenia at $87 \mathrm{y}$ : OR: $0.47,95 \% \mathrm{Cl}$ : $0.17,1.28, \mathrm{p}=\mathrm{NS}$

$\mathrm{mHDI}$ scores (continuous) at $71 \mathrm{y}$ and sarcopenia at $87 \mathrm{y}$ : OR: $0.90,95 \% \mathrm{Cl}$ $0.63,1.27, p=N S$

mMDS score (high vs. low; medium vs. low) at $71 \mathrm{y}$ and sarcopenia at $87 \mathrm{y}$ : OR: $0.33,95 \% \mathrm{Cl}: 0.09,1.23, \mathrm{p}=\mathrm{NS}$; OR: $0.50,95 \% \mathrm{Cl}: 0.22,1.16, p=N S$

\section{Key confounders accounted for:}

Sex; Age; SES; Anthropometry; Dietary protein intake; Physical activity

Other: Smoking; Inflammation (CRP); Hospital stay

\section{Limitations:}

- Did not account for the following key confounders: Race/ethnicity; Physical disability

- Excluded subjects with $>10 \%$ weight loss between 60-70y; exposure occurred prior to start of $\mathrm{f} / \mathrm{u}$

- Only assessed dietary intake once at baseline, and document instability in dietary intake over the course of the study; were not able to not account for possible changes in dietary intake over f/u

- Did not account for missing data

- No published protocol; potential for selective outcome reporting

\section{Funding Sources:}

Swedish Medical Research Council, Uppsala University

Summary: Higher mHDI score (medium vs. low) at 71y was significantly associaeted with lower risk of sarcopenia at 87y.

However, high vs. low score and analysis of scores continuously were not 
significantly associated with risk of sarcopenia.

Higher mMDS score (continuous) at 71y was associated with lower risk of

sarcopenia at 87y. However, when mMDS scores were analyzed categorically (low

vs. medium or high), there were no significantly associations with risk of sarcopenia. 
Table 2: Summary of the results from studies that examined the relationship between dietary patterns and diets based on macronutrient distribution and sarcopenia ${ }^{\text {viii }}$

\begin{tabular}{|c|c|c|}
\hline $\begin{array}{l}\text { Article, Study } \\
\text { Design, Country }\end{array}$ & Significant Results & Non-Signigicant Results \\
\hline $\begin{array}{l}\text { Chan, } 2016^{1} \\
\text { PCS } \\
\text { Hong Kong, } \\
\text { China }\end{array}$ & $\begin{array}{l}\text { Dietary Quality Index-International score (DQI-I) } \\
\text { - Positive components: Vegetables, fruit, grain, fiber, protein, } \\
\text { iron, calcium, vitamin C, overall food group variety, within } \\
\text { food group variety } \\
\text { - Moderation components: Total fat, SFA, cholesterol, sodiu, } \\
\text { empty calorie foods, macronutrient ratio, fatty acid ratio }\end{array}$ & $\begin{array}{l}\text { Men: OR: } 1.00,95 \% \mathrm{Cl}: 0.98 \\
\text { 1.02, } p=0.670, \mathrm{NS} \\
\text { Women: OR: } 0.99, \mathrm{Cl}: 0.97,1.02 \\
p=0.611, \mathrm{NS}\end{array}$ \\
\hline & $\begin{array}{l}\text { Mediterranean Diet Score (MDS) } \\
\text { - Positive components: Vegetables, legumes, fruit and nuts, } \\
\text { cereals, fish, MUFA:SFA ratio } \\
\text { - Negative components: Red and processed meat, dairy } \\
\text { products } \\
\text { - Moderation components: Alcohol }\end{array}$ & $\begin{array}{l}\text { Men: OR: } 0.98,95 \% \mathrm{Cl}: 0.86 \\
1.10, \mathrm{P}=0.678, \mathrm{NS} \\
\text { Women: OR: } 0.96, \mathrm{Cl}: 0.83,1.11 \\
p=0.602, \mathrm{NS}\end{array}$ \\
\hline & $\begin{array}{l}\text { "Vegetables-Fruits": Higher in tomatoes, dark green and leafy } \\
\text { vegetables, cruciferous vegetables, starchy vegetables, other } \\
\text { vegetables, soy, fruits, legumes, mushroom and fungi, and } \\
\text { lower in fats and oils }\end{array}$ & $\begin{array}{l}\text { Men: OR: } 1.07,95 \% \mathrm{Cl}: 0.88 \\
1.29, p=0.522, \mathrm{NS} \\
\text { Women: OR: } 1.15, \mathrm{Cl}: 0.94,1.40 \\
p=0.190, \mathrm{NS}\end{array}$ \\
\hline & $\begin{array}{l}\text { "Snacks-Drinks-Milk Products": Higher in condiments, coffee, } \\
\text { fast food, nuts, French fries and potato chips, milk and milk } \\
\text { products, whole grains, sweets and desserts, beverage }\end{array}$ & $\begin{array}{l}\text { Men: OR: } 0.95, \mathrm{Cl}: 0.78,1.16 \\
p=0.611, \mathrm{NS} \\
\text { Women: OR: } 1.20, \mathrm{Cl}: 0.94 \\
1.53, p=0.148, \mathrm{NS}\end{array}$ \\
\hline & $\begin{array}{l}\text { "Meat-Fish": Higher in dim sum, red and processed meats, } \\
\text { poultry, fish and seafood, wine, and lower in refined grains }\end{array}$ & $\begin{array}{l}\text { Men: OR: } 1.00,95 \% \mathrm{Cl}: 0.84 \\
\text { 1.20, } p=1.000, \mathrm{NS} \\
\text { Women: OR: } 1.10, \mathrm{Cl}: 0.87,1.40 \\
p=0.415, \mathrm{NS}\end{array}$ \\
\hline
\end{tabular}

viii Abbreviations: AMDR, Acceptable macronutrient distribution range; C, Carbohydrate; DQI-I, Diet quality index - international; EWGSOP, European Working Group on

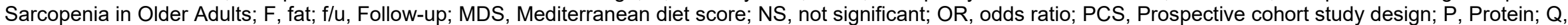
Quantile (quintile or quartile as appropriate); RR, Relative risk; SES, Socioeconomic status 


\begin{tabular}{|c|c|c|c|}
\hline $\begin{array}{l}\text { Article, Study } \\
\text { Design, Country }\end{array}$ & Dietary Patterns & Significant Results & Non-Signigicant Results \\
\hline \multirow[t]{3}{*}{$\begin{array}{l}\text { Granic, } 2019^{2} \\
\text { PCS } \\
\text { United Kingdom }\end{array}$} & $\begin{array}{l}\text { - 'Low Red Meat': lower in legumes, sweets and desserts, and } \\
\text { higher in fish/seafood; macronutrient distribution: } 47 \% \mathrm{C} \text {, } \\
37 \% \mathrm{~F}, 16 \% \mathrm{P}\end{array}$ & $\begin{array}{l}\text { 'Traditional British' vs. 'Low Butter': } \\
\text { OR: } 2.42,95 \% \mathrm{Cl}: 1.15,5.09 \\
\mathrm{p}=0.02 \text {. }\end{array}$ & $\begin{array}{l}\text { 'Low Red Meat' vs. 'Low Butter": } \\
\text { OR: } 1.77,95 \% \mathrm{Cl}: 0.84,3.74 \text {, } \\
p=0.13, \text { NS }\end{array}$ \\
\hline & $\begin{array}{l}\text { - 'Traditional British': higher in butter, red meats and meat } \\
\text { dishes, gravy, potatoes and potato dishes, vegetables, and } \\
\text { sweets/desserts; macronutrient distribution: } 46 \% \text { C, } 39 \% \\
\text { F,15\% P }\end{array}$ & $\begin{array}{l}\text { Protein intake } \geq 1.0 \mathrm{~g} / \mathrm{kg} \mathrm{BW} / \mathrm{d} \text { : OR: } \\
3.62,95 \% \mathrm{Cl}: 1.33,9.88, \mathrm{p}=0.01\end{array}$ & $\begin{array}{l}\text { 'Low Red Meat' vs. 'Low Butter": } \\
\text { OR: } 1.05,95 \% \mathrm{Cl}: 0.37,3.03 \text {, } \\
\mathrm{p}=0.92, \mathrm{NS} \text {. }\end{array}$ \\
\hline & $\begin{array}{l}\text { - 'Low Butter': Higher in unsaturated fat spreads and oils; } \\
\text { macronutrient distribution: } 48 \% \mathrm{C}, 35 \% \mathrm{~F}, 17 \% \mathrm{P}\end{array}$ & & $\begin{array}{l}\text { 'Traditional British' vs. 'Low } \\
\text { Butter': OR: } 1.67,95 \% \mathrm{Cl}: 0.59 \text {, } \\
\text { 4.67, } p=0.33, \mathrm{NS}\end{array}$ \\
\hline \multirow{4}{*}{$\begin{array}{l}\text { Isanejad, } 2018^{3} \\
\text { PCS } \\
\text { Finland }\end{array}$} & Baltic Sea Diet Score (BSD) & - Q1: OR: 1.00 & \multirow{2}{*}{$\begin{array}{l}\text { Continuous: OR: }-0.03(0.01) \\
p=0.512\end{array}$} \\
\hline & $\begin{array}{l}\text { - Positive components: Total fruits (mostly apples, pears) and } \\
\text { berries, vegetables (root vegetables, legumes, nuts, } \\
\text { mushrooms, and vegetable products, and excluding } \\
\text { potatoes), fiber from cereal products, fish, low-fat milk, } \\
\text { PUFA: SFA ratio } \\
\text { - Negative components: Processed meat products, total fat } \\
\text { intake, alcohol } \\
\text { - Macronutrient distribution of \% energy for BSD quartiles: } \\
\circ \text { Q1: } 47.7 \% \text { C, } 34.9 \% \mathrm{~F}, 15.9 \% \mathrm{P} \\
\circ \mathrm{Q} 2: 48.3 \% \mathrm{C}, 32.1 \% \mathrm{~F}, 16.7 \% \mathrm{P} \\
\circ \mathrm{Q}: 49.9 \% \mathrm{C}, 41.1 \% \mathrm{~F}, 13.1 \% \mathrm{P} \\
\circ \mathrm{Q} 4: 50.8 \% \mathrm{C}, 27.1 \% \mathrm{~F}, 18.5 \% \mathrm{P}\end{array}$ & $\begin{array}{l}\text { - Q2: OR: } 0.82,95 \% \mathrm{Cl}: 0.30,2.24 \\
\text { - Q3: OR: } 0.76,95 \% \mathrm{Cl}: 0.27,2.13 \\
\text { - Q4: OR: } 0.33,95 \% \mathrm{Cl}: 0.13,0.79 \\
\text { - } \mathrm{p}=0.015\end{array}$ & \\
\hline & Mediterranean Diet Score (MDS) & & - Q1: OR: 1.00 \\
\hline & $\begin{array}{l}\text { - Positive components: Total fruits (mostly apples, pears) and } \\
\text { berries, vegetables (root vegetables, legumes and nuts, } \\
\text { mushrooms, and vegetable products), cereals and potatoes, } \\
\text { fish, PUFA+MUFA:SFA ratio } \\
\text { - Negative components: Meat (including sausage, eggs), milk } \\
\text { and dairy products } \\
\text { - Moderation component: Alcohol } \\
\text { - Macronutrient distribution of \% energy for MDS quartiles: } \\
\circ \text { Q1: } 48.4 \% \text { C, 31.7\% F, } 17.5 \% \text { P } \\
\circ \text { Q2: } 49.1 \% \text { C, 30.8\% F, } 17.8 \% \text { P } \\
\circ \text { Q3: } 49.7 \% \text { C, } 31.1 \% \mathrm{~F}, 17.2 \% \mathrm{P}\end{array}$ & & $\begin{array}{l}\text { - Q2: OR: } 0.87,95 \% \mathrm{Cl}: 0.33 \\
2.25 \\
\text { - Q3: OR: } 1.20,95 \% \mathrm{Cl}: 0.44 \\
\text { 3.23 } \\
\text { - Q4: OR: } 0.75,95 \% \mathrm{Cl}: 0.28 \\
2.05 \\
\text { - } p=0.751 \\
\text { Continuous: OR: }-0.03(0.02) \\
p=0.67\end{array}$ \\
\hline
\end{tabular}




\begin{tabular}{|c|c|c|c|}
\hline $\begin{array}{l}\text { Article, Study } \\
\text { Design, Country }\end{array}$ & Dietary Patterns & Significant Results & Non-Signigicant Results \\
\hline \multicolumn{4}{|c|}{ OQ4: $51.4 \%$ C, $29.9 \%$ F, $17.1 \% \mathrm{P}$} \\
\hline \multirow{6}{*}{$\begin{array}{l}\text { Karlsson, } 2019^{4} \\
\text { PCS } \\
\text { Sweden }\end{array}$} & Modified Healthy Diet Indicator (mHDI) & \multirow{3}{*}{$\begin{array}{l}\text { Medium vs. Low: OR: } 0.38,95 \% \mathrm{Cl}: \\
0.15,0.97\end{array}$} & \multirow{2}{*}{$\begin{array}{l}\text { High vs. Low: OR: } 0.47,95 \% \mathrm{Cl} \text { : } \\
0.17,1.28, p=\mathrm{NS}\end{array}$} \\
\hline & $\begin{array}{l}\text { - Positive components: PUFA, protein, carbohydrates, fiber, } \\
\text { fruit and vegetables, fish }\end{array}$ & & \\
\hline & - Negative components: Sucrose, SFA, cholesterol & & $\begin{array}{l}\text { Continuous: OR: } 0.90,95 \% \mathrm{Cl} \\
0.63,1.27, p=\mathrm{NS}\end{array}$ \\
\hline & Modified Mediterranean Diet Score (mMDS) & \multirow{3}{*}{$\begin{array}{l}\text { Continuous: OR: } 0.49,95 \% \mathrm{Cl}: 0.31 \text {, } \\
0.80\end{array}$} & \multirow{3}{*}{$\begin{array}{l}\text { Medium vs. Low: OR: } 0.33,95 \% \\
\text { Cl: } 0.09,1.23, p=N S \\
\text { High vs. Low: OR: } 0.50,95 \% \mathrm{Cl}: \\
0.22,1.16, p=N S\end{array}$} \\
\hline & - Positive components: PUFA:SFA ratio, vegetables and & & \\
\hline & $\begin{array}{l}\text { legumes, fruit and berries, cereals and potatoes, fish } \\
\text { milk products }\end{array}$ & & \\
\hline
\end{tabular}


Table 3. Risk of bias for observational studies examining dietary patterns and diets based on macronutrient distribution and sarcopenia ${ }^{\mathrm{ix}, \mathrm{x}}$

\begin{tabular}{|c|c|c|c|c|c|c|c|}
\hline Article & Confounding & $\begin{array}{l}\text { Selection of } \\
\text { participants }\end{array}$ & $\begin{array}{l}\text { Classification } \\
\text { of exposures }\end{array}$ & $\begin{array}{l}\text { Deviations } \\
\text { from intended } \\
\text { exposures }\end{array}$ & Missing data & $\begin{array}{c}\text { Outcome } \\
\text { measurement }\end{array}$ & $\begin{array}{l}\text { Selection of } \\
\text { the reported } \\
\text { result }\end{array}$ \\
\hline Chan, $2016^{1}$ & Serious & Serious & Low & Serious & Moderate & Low & Moderate \\
\hline Granic, 2019² & Serious & Moderate & Low & Serious & Moderate & Low & Moderate \\
\hline Isanejad, $2018^{3}$ & Serious & Serious & Low & Serious & Moderate & Moderate & Serious \\
\hline Karlsson, $2019^{4}$ & Serious & Moderate & Low & Critical & Serious & Low & Moderate \\
\hline
\end{tabular}

\footnotetext{
${ }^{i x}$ A detailed description of the methodology used for assessing risk of bias is available on the NESR website: https://nesr.usda.gov/2020-dietaryquidelines-advisory-committee-systematic-reviews and in Part C of the following reference: Dietary Guidelines Advisory Committee. 2020. Scientific Report of the 2020 Dietary Guidelines Advisory Committee: Advisory Report to the Secretary of Agriculture and the Secretary of Health and Human Services. U.S. Department of Agriculture, Agricultural Research Service, Washington, DC.

× Possible ratings of low, moderate, serious, critical, or no information determined using the "Risk of Bias for Nutrition Observational Studies" tool (RoBNObs) (Dietary Guidelines Advisory Committee. 2020. Scientific Report of the 2020 Dietary Guidelines Advisory Committee: Advisory Report to the Secretary of Agriculture and the Secretary of Health and Human Services. U.S. Department of Agriculture, Agricultural Research Service, Washington, DC.)
} 


\section{METHODOLOGY}

The NESR team used its rigorous, protocol-driven methodology to support the 2020 Dietary Guidelines Advisory Committee in conducting this systematic review.

NESR's systematic review methodology involves:

- Developing a protocol,

- Searching for and selecting studies,

- Extracting data from and assessing the risk of bias of each included study,

- Synthesizing the evidence,

- Developing conclusion statements,

- Grading the evidence underlying the conclusion statements, and

- Recommending future research.

A detailed description of the methodology used in conducting this systematic review is available on the NESR website: https://nesr.usda.gov/2020-dietary-guidelines-advisory-committee-systematicreviews, and can be found in the 2020 Dietary Guidelines Advisory Committee Report, Part C: Methodology. ${ }^{\mathrm{xi}}$ This systematic review was peer reviewed by Federal scientists, and information about the peer review process can also be found in the Committee's Report, Part C: Methodology. Additional information about this systematic review, including a description of and rationale for any modifications made to the protocol can be found in the 2020 Dietary Guidelines Advisory Committee Report, Chapter 8. Dietary Patterns.

Below are details of the final protocol for the systematic review described herein, including the:

- Analytic framework

- Literature search and screening plan

- Literature search and screening results

xi Dietary Guidelines Advisory Committee. 2020. Scientific Report of the 2020 Dietary Guidelines Advisory Committee: Advisory Report to the Secretary of Agriculture and the Secretary of Health and Human Services. U.S. Department of Agriculture, Agricultural Research Service, Washington, DC. 


\section{ANALYTIC FRAMEWORK}

The analytic framework (Figure 1) illustrates the overall scope of the systematic review, including the population, the interventions and/or exposures, comparators, and outcomes of interest. It also includes definitions of key terms and identifies key confounders and other factors considered in the systematic review. The inclusion and exclusion criteria that follow provide additional information about how parts of the analytic framework were defined and operationalized for the review.

\section{Figure 1: Analytic framework}

Systematic review question: What is the relationship between dietary patterns consumed and sarcopenia?

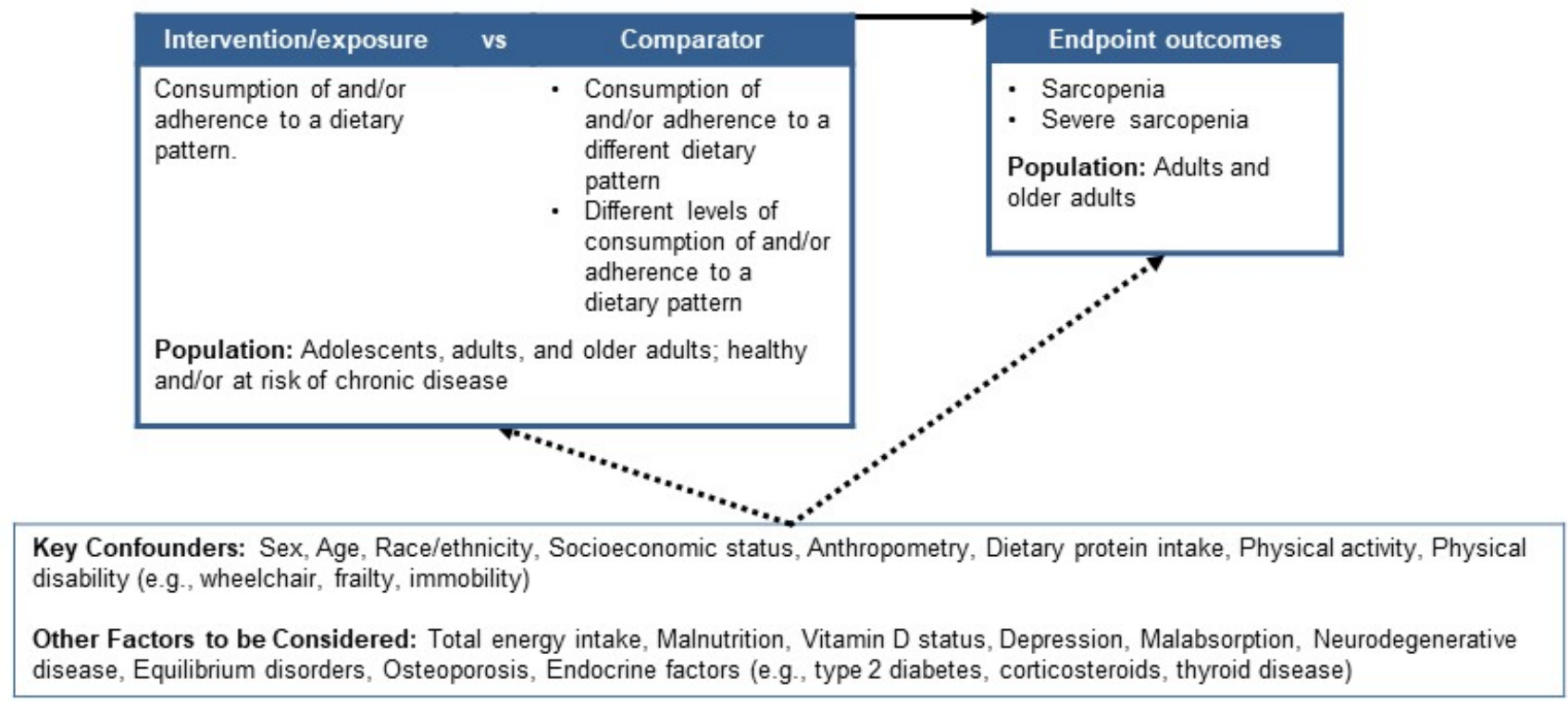

Key definitions

Dietary patterns - The quantities, proportions, variety, or combination of different foods, drinks, and nutrients (when available) in diets, and the frequency with which they are habitually consumed

Legend

Sarcopenia - A progressive and generalized loss of skeletal muscle mass, alone or in conjunction with either or both low muscle strength and low muscle performance. 


\section{LITERATURE SEARCH AND SCREENING PLAN}

\section{Inclusion and exclusion criteria}

This table provides the inclusion and exclusion criteria for the systematic review. The inclusion and exclusion criteria are a set of characteristics used to determine which articles identified in the literature search were included in or excluded from the systematic review.

\section{Table 4. Inclusion and exclusion criteria}

Category Inclusion Criteria Exclusion Criteria

\section{Study} design

Intervention/ exposure
- Randomized controlled trials

- Non-randomized controlled trials, including quasiexperimental and controlled before and after studies

- Prospective cohort studies

- Retrospective cohort studies

- Nested case-control studies

- Studies that examine consumption of and/or adherence to a

1. Dietary pattern [i.e., the quantities, proportions, variety, or combination of different foods, drinks, and nutrients (when available) in diets, and the frequency with which they are habitually consumed]), including, at a minimum, a description of the foods and beverages in the pattern

- Dietary patterns may be measured or derived using a variety of approaches, such as adherence to a priori patterns (indices/scores), data driven patterns (factor or cluster analysis), reduced rank regression, or other methods, including clinical trials

and/or

2. Diet based on macronutrient distribution outside of the AMDR and

0 include the macronutrient distribution of carbohydrate, fat, and protein of the diet, and

- include at least one macronutrient outside of the acceptable macronutrient distribution range (AMDR) $)^{\text {xii, } \text {, xii }}$
- Uncontrolled trials

- Case-control studies

- Cross-sectional studies

- Uncontrolled before-and-after studies

- Narrative reviews

- Systematic reviews

- Meta-analyses

- Studies that

1a. do not provide a description of the dietary pattern, which at minimum, must include the foods and beverages in the pattern (i.e., studies that examine a labeled dietary patterns, but do not describe the foods and beverages consumed)

2a. Examine consumption of and/or adherence to a diet based on macronutrient proportion in which all macronutrients are within the AMDR.

$2 b$. Do not describe the entire macronutrient distribution of the diet (i.e., studies that only examine a single macronutrient in relation to outcomes)

xii Institute of Medicine. Dietary Reference Intakes for Energy, Carbohydrate, Fiber, Fat, Fatty Acids, Cholesterol, Protein, and Amino Acids. Washington (DC): The National Academies Press; 2002.

xiii Macronutrient percent of energy outside of the AMDR are as follows:

- Carbohydrate for all age groups: $<45$ or $>65$ percent of energy;

- Protein for children, 1-3y: $<5$ or $>20$ percent of energy, Protein for children, $4-18 y$ : $<10$ or $>30$ percent of energy, Protein for adults, age $19 \mathrm{y}$ and older: $<10$ or $>35$ percent of energy;

- Fat for children, 1-3y: $<30$ or $>40$ percent of energy, Fat for children, $4-18 y:<25$ or $>35$ percent of energy, Fat for adults, age $19 y$ and older: $<20$ or $>35$ percent of energy. 


\section{Category Inclusion Criteria Exclusion Criteria}

Comparator - Dietary patterns described by foods and beverages N/A consumed:

- Consumption of and/or adherence to a different dietary pattern

- Different levels of consumption of and/or adherence to a dietary pattern

- Diets described by macronutrient distribution

- Different macronutrient distribution of carbohydrate, fat, and protein

\begin{tabular}{|c|c|c|}
\hline Outcomes & Studies that report sarcopenia or severe sarcopenia & $N / A$ \\
\hline $\begin{array}{l}\text { Date of } \\
\text { publication }\end{array}$ & January 2000 - October 2019 & $\begin{array}{l}\text { Articles published prior to January } 2000 \text { or after } \\
\text { October } 2019\end{array}$ \\
\hline $\begin{array}{l}\text { Publication } \\
\text { status }\end{array}$ & Articles that have been peer-reviewed & $\begin{array}{l}\text { Articles that have not been peer-reviewed and are } \\
\text { not published in peer-reviewed journals, including } \\
\text { unpublished data, manuscripts, pre-prints, reports, } \\
\text { abstracts, and conference proceedings }\end{array}$ \\
\hline $\begin{array}{l}\text { Language of } \\
\text { publication }\end{array}$ & Articles published in English & Articles published in languages other than English \\
\hline Country & $\begin{array}{l}\text { Studies conducted in countries ranked as high or higher } \\
\text { human development }\end{array}$ & $\begin{array}{l}\text { Studies conducted in countries ranked as medium or } \\
\text { lower human development }\end{array}$ \\
\hline $\begin{array}{l}\text { Study } \\
\text { participants }\end{array}$ & $\begin{array}{l}\text { - } \text { Human participants } \\
\text { - } \text { Males } \\
\text { - } \quad \text { Females }\end{array}$ & $\begin{array}{l}\text { - Non-human participants (i.e., animals) } \\
\text { - Women during pregnancy and lactation }\end{array}$ \\
\hline $\begin{array}{l}\text { Age of study } \\
\text { participants }\end{array}$ & $\begin{array}{l}\text { - Age at intervention or exposure: } \\
\circ \text { Adolescents (ages 12-18 years) } \\
\circ \text { Adults (ages 19-64 years) } \\
\circ \quad \text { Older adults (ages } 65 \text { years and older) } \\
\text { - Age at outcome: } \\
\circ \quad \text { Adults (ages 19-64 years) } \\
\circ \quad \text { Older adults (ages } 65 \text { years and older) }\end{array}$ & $\begin{array}{l}\text { - Age at intervention or exposure: } \\
\circ \text { Infants and toddlers (birth to } 24 \text { months) } \\
\circ \text { Children (ages 2-12 years) } \\
\text { - Age at outcome: } \\
\circ \text { Infants and toddlers (birth to } 24 \text { months) } \\
\circ \text { Children and adolescents (ages 2-18 years) }\end{array}$ \\
\hline
\end{tabular}

xiv The Human Development classification was based on the Human Development Index (HDI) ranking from the year the study intervention occurred or data were collected (UN Development Program. HDI 1990-2017 HDRO calculations based on data from UNDESA (2017a), UNESCO Institute for Statistics (2018), United Nations Statistics Division (2018b), World Bank (2018b), Barro and Lee (2016) and IMF (2018). Available from:

http://hdr.undp.org/en/data). If the study did not report the year in which the intervention occurred or data were collected, the HDI classification for the year of publication was applied. HDI values are available from 1980, and then from 1990 to present. If a study was conducted prior to 1990, the HDI classification from 1990 was applied. If a study was conducted in 2018 or 2019, the most current HDI classification was applied. When a country was not included in the HDI ranking, the current country classification from the World Bank was used instead (The World Bank. World Bank country and lending groups. Available from: https://datahelpdesk.worldbank.org/knowledgebase/articles/906519-world-country-and-lending-groups). 


\begin{tabular}{lll}
\hline Category & Inclusion Criteria & Exclusion Criteria \\
\hline $\begin{array}{l}\text { Health } \\
\text { status of } \\
\text { study } \\
\text { participants }\end{array}$ & $\begin{array}{l}\text { Studies that enroll participants who are healthy } \\
\text { and/or at risk for chronic disease, including those } \\
\text { with obesity }\end{array}$ & $\begin{array}{l}\text { Studies that exclusively enroll participants } \\
\text { diagnosed with a disease. (For this criterion, } \\
\text { studies that exclusively enroll subjects with } \\
\text { obesity will be included.) }\end{array}$ \\
& $\begin{array}{l}\text { Studies that enroll some participants diagnosed } \\
\text { with a disease or with sarcopenia or severe } \\
\text { sarcopenia }\end{array}$ & $\begin{array}{l}\text { Studies that exclusively enroll participants } \\
\text { diagnosed with sarcopenia, or severe sarcopenia } \\
\text { (i.e., studies that aim to treat participants who } \\
\text { have already been diagnosed with the outcome } \\
\text { of interest) }\end{array}$ \\
\end{tabular}




\section{Electronic databases and search terms}

Listed below are the databases searched to identify all potentially relevant articles that have been published to address the systematic review question. A search strategy was developed and implemented in three databases (PubMed, Cochrane Central Register of Controlled Trials (CENTRAL), and Embase) to conduct this systematic review.

\section{PubMed}

- Provider: U.S. National Library of Medicine

- Date(s) Searched: October 8, 2019

- Date range searched: January 1, 2000-October 8, 2019

- Search Terms:

\#1 - dietary pattern* OR diet pattern* OR eating pattern* OR food pattern* OR diet quality* OR eating habit* OR dietary habit* OR diet habit* OR food habit* OR beverage habit* OR "Feeding Behavior"[Mesh:NoExp] OR dietary profile* OR food profile* OR diet profile* OR eating profile* OR dietary guideline* OR dietary recommendation* OR dietary intake* OR eating style* OR "Diet, Mediterranean"[Mesh] OR Mediterranean Diet*[tiab] OR "Dietary Approaches To Stop

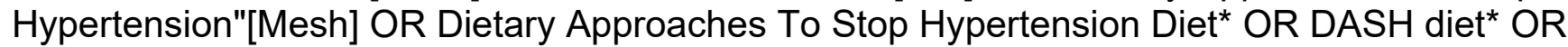

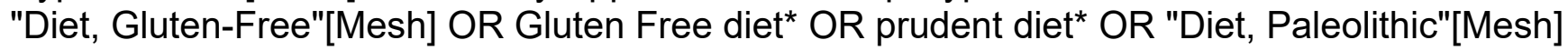
OR Paleolithic Diet* OR "Diet, Vegetarian"[Mesh] OR vegetarian $\operatorname{diet}^{*}\left[\right.$ tiab] OR vegan $\operatorname{diet}^{*}$ OR "Diet, Healthy"[Mesh] OR plant based diet* OR "Diet, Western"[Mesh] OR western diet* OR "Diet, Carbohydrate-Restricted"[Mesh] OR low-carbohydrate diet* OR high carbohydrate diet* OR Ketogenic Diet* OR Nordic Diet* OR "Diet, Fat-Restricted"[Mesh] OR "Diet, HighFat"[Mesh] OR "Diet, High-Protein"[Mesh] OR high protein diet*[tiab] OR protein intake* OR high-fat diet* OR low fat diet* OR "Diet, Protein-Restricted"[Mesh] OR low protein diet $^{*}$ OR "Diet, Sodium-Restricted"[Mesh] OR low-sodium diet* OR low salt diet* OR (("Dietary Proteins"[Mesh] OR dietary protein*[tiab] OR "Dietary Carbohydrates"[Mesh] OR dietary carbohydrate*[tiab] OR "Dietary Fats"[Mesh] OR dietary fat*[tiab] OR hypocaloric OR hypocaloric) AND (diet[tiab] OR diets[tiab] OR consumption[tiab] OR intake[tiab] OR supplement*[tiab])) OR (("Guideline Adherence"[Mesh] OR guideline adherence $\left.{ }^{*}\right)$ AND (diet[tiab] OR dietary[tiab] OR food[tiab] OR beverage*[tiab] OR nutrition*[tiab])) OR diet score* OR diet quality score* OR diet quality index* OR kidmed OR diet index* OR dietary index* OR food score* OR MedDietScore OR healthy eating index[tiab] OR ((pattern[tiab] OR patterns[tiab] OR consumption[tiab] OR habit"[tiab]) AND ("Diet"[Mesh:NoExp] OR diet[tiab] OR diets[tiab] OR dietary[tiab] OR "Food"[Mesh] OR food[tiab] OR foods[tiab] OR "Beverages"[Mesh] OR beverage[tiab] OR beverages[tiab]))

\#2 - "Sarcopenia"[Mesh] OR sarcopen* OR presarcopen* OR "Muscular Atrophy"[Mesh:NoExp] OR muscular atroph* OR "Muscle Fatigue"[Mesh] OR muscle fatigue[tiab] OR "Muscle Strength"[Mesh] OR muscle wast* OR muscle weak* OR muscle loss* OR muscle function* OR hand strength* OR grip strength* OR handgrip* OR pinch strength* OR "Physical Functional Performance"[Mesh] OR physical performance* OR "Psychomotor Performance"[Mesh] OR psychomotor performance[tiab] OR ((muscle[tiab] OR muscles[tiab] OR "Muscles"[Mesh] OR "Muscle, Skeletal"[Mesh]) AND ("Fatigue"[Mesh] OR fatigue[tiab] OR weakness[tiab] OR "Frailty"[Mesh] OR frail[tiab] OR frailty[tiab] OR "Geriatric Assessment"[Mesh] OR geriatric*[tiab] OR "Osteoporosis"[Mesh] OR osteoporos*[tiab] OR "Age Factors"[Mesh] OR "Aging"[Mesh] OR "Obesity"[Mesh] OR obesity[tiab] OR "Body Mass Index"[Mesh] OR body mass index[tiab] OR BMI[tiab] OR lean mass[tiab] OR fat mass[tiab] OR "Activities of Daily Living"[Mesh] OR "Quality of Life"[Mesh] OR quality of life[tiab] OR 
"Postural Balance"[Mesh] OR posture*[tiab] OR "Physical Exertion"[Mesh] OR physical exertion*[tiab] OR "Mobility Limitation"[Mesh] OR mobility limitation* OR "Motor Activity"[Mesh] OR motor activit*[tiab] OR "Walking"[Mesh] OR walking[tiab] OR "Gait"[Mesh] OR gait[tiab])) OR (("Aging"[Mesh] OR older adult*[tiab]) AND (physical activit[tiab] OR physical function[tiab] OR physical impairment[tiab] OR physical performance[tiab] OR functional limit* ${ }^{*}$ tiab] OR functional decline[tiab] OR "Mobility Limitation"[Mesh] OR mobility limitation* OR "Walking"[Mesh] OR walking[tiab] OR "Activities of Daily Living"[Mesh] OR activities of daily living[tiab] OR "Independent Living"[Mesh] OR independent living[tiab] OR "Quality of Life"[Mesh] OR quality of life[tiab]))

\section{\#3 - (\#1 AND \#2)}

\#4 - (\#1 AND \#2) NOT ("Animals"[Mesh] NOT ("Animals"[Mesh] AND "Humans"[Mesh])) NOT (editorial[ptyp] OR comment[ptyp] OR news[ptyp] OR letter[ptyp] OR review[ptyp] OR systematic review[ptyp] OR systematic review[ti] OR meta-analysis[ptyp] OR meta-analysis[ti] OR meta-analyses[ti] OR retracted publication[ptyp] OR retraction of publication[ptyp] OR retraction of publication[tiab] OR retraction notice[ti]) Filters: Publication date from 2000/01/01 to 2019/10/08; English

\section{Cochrane Central Register of Controlled Trials (CENTRAL)}

- Provider: John Wiley \& Sons

- Date(s) Searched: October 8, 2019

- Date range searched: January 1, 2000-October 8, 2019

- Search Terms:

\#1 - ("dietary pattern" OR "diet pattern" OR "eating pattern*" OR "food pattern"” OR "diet quality" OR "eating habit" OR "dietary habit" OR "diet habit" OR "food habit" OR "beverage habit"” OR [mh ^"Feeding Behavior"] OR "dietary profile*" OR "food profile*" OR "diet profile*" OR "eating profile*" OR "dietary guideline *" OR "dietary recommendation*" OR "dietary intake" OR "eating style" OR [mh "Diet, Mediterranean"] OR "Mediterranean Diet" OR [mh "Dietary Approaches To Stop Hypertension"] OR "Dietary Approaches To Stop Hypertension Diet*" OR "DASH diet"” OR [mh "Diet, Gluten-Free"] OR "Gluten Free diet"” OR "prudent diet" OR [mh "Diet, Paleolithic"] OR "Paleolithic Diet"” OR [mh "Diet, Vegetarian"] OR "vegetarian diet" OR "vegan diet" OR [mh "Diet, Healthy"] OR "plant based diet*" OR [mh "Diet, Western"] OR "western diet*" OR [mh "Diet, Carbohydrate-Restricted"] OR "low-carbohydrate diet" OR "high carbohydrate diet*" OR "Ketogenic Diet*" OR "Nordic Diet*" OR [mh "Diet, Fat-Restricted"] OR [mh "Diet, High-Fat"] OR [mh "Diet, High-Protein"] OR "high protein diet*" OR "protein intake*" OR "high-fat diet"” OR "low fat diet*" OR [mh "Diet, Protein-Restricted"] OR "low protein diet"” OR [mh "Diet, Sodium-Restricted"] OR “low-sodium diet"” OR "low salt diet"):ti, ab,kw

\#2 - (([mh "Dietary Proteins"] OR "dietary protein*” OR [mh "Dietary Carbohydrates"] OR "dietary carbohydrate*" OR [mh "Dietary Fats"] OR "dietary fat*" OR hypocaloric OR hypocaloric) NEAR/6 (diet OR diets OR consumption OR intake OR supplement*))

\#3 - ([mh "Guideline Adherence"] OR guideline adherence*) NEAR/6 (diet OR dietary OR food OR beverage* OR nutrition*))

\#4 - ("diet score*" OR "diet quality score*" OR "diet quality index*" OR kidmed OR "diet index" OR "dietary index" OR "food score*" OR MedDietScore OR "healthy eating index"):ti,ab,kw

\#5 - (( (pattern OR patterns OR consumption OR habit*) NEAR/6 ([mh ^ Diet] OR diet OR diets 
OR dietary OR [mh Food] OR food OR foods OR [mh Beverages] OR beverage OR beverages))):ti,ab,kw

\section{\#6 - \#1 OR \#2 OR \#3 OR \#4 OR \#5}

\#7 - [mh Sarcopenia] OR [mh "Muscular Atrophy"] OR [mh "Muscle Fatigue"] OR [mh "Muscle Strength"] OR [mh "Physical Functional Performance"] OR [mh "Psychomotor Performance"]

\#8 - (sarcopen* OR presarcopen* OR "muscular atroph"” OR "muscle fatigue" OR "muscle wast" OR "muscle weak" OR "muscle loss *" OR "muscle function*" OR "hand strength" OR "grip strength" OR handgrip OR "pinch strength" OR "physical performance*" OR "psychomotor performance"):ti,ab,kw

\#9 - ((muscle OR muscles OR [mh Muscles] OR [mh "Muscles, Skeletal"]) NEAR/3 ([mh Fatigue] OR fatigue OR weakness OR [mh Frailty] OR frail OR frailty OR [mh "Geriatric Assessment"] OR geriatric* OR [mh Osteoporosis] OR osteoporos* OR [mh "Age Factors"] OR [mh Aging] OR [mh Obesity] OR obesity OR [mh "Body Mass Index"] OR "body mass index" OR BMI OR "lean mass" OR "fat mass" OR [mh "Activities of Daily Living"] OR [mh "Quality of Life"] OR "quality of life" OR [mh "Postural Balance"] OR posture* OR [mh "Physical Exertion"] OR "physical exertion*” OR [mh "Motor Activity"] OR "motor activit"” OR [mh "Walking"] OR walking OR [mh Gait] OR gait)):ti,ab,kw

\#10 - (([mh Aging] OR "older adult") NEAR/6 ("physical activit"” OR "physical function" OR "physical impairment" OR "functional limit"” OR "functional decline" OR [mh "Mobility Limitation"] OR "mobility limitation*” OR [mh Walking] OR walking OR [mh "Activities of Daily Living"] OR "activities of daily living" OR [mh "Independent Living"] OR "independent living" OR [mh "Quality of Life"] OR "quality of life"))

\#11 - \#7 OR \#8 OR \#9 OR \#10

\#12 - \#6 AND \#11" with Publication Year from 2000 to 2019 in Trials (Word variations have been searched)

\section{Embase}

- Provider: Elsevier

- Date(s) Searched: October 8, 2019

- Date range searched: January 1, 2000-October 8, 2019

- Search Terms:

\#1 - 'feeding behavior'/de OR 'mediterranean diet'/exp OR 'dash diet'/exp OR 'gluten free diet'/exp OR 'paleolithic diet'/de OR 'vegetarian diet'/exp OR 'healthy diet'/de OR 'western diet'/de OR 'low carbohydrate diet'/exp OR 'low fat diet'/de OR 'lipid diet'/exp OR 'protein diet'/exp OR 'protein restriction'/de OR 'sodium restriction'/de

\#2 - 'dietary pattern*':ab,ti OR 'diet pattern*':ab,ti OR 'eating pattern': ab,ti OR 'food pattern'”:ab,ti OR 'diet quality":ab,ti OR 'eating habit':ab,ti OR 'dietary habit': ab,ti OR 'diet habit':ab,ti OR 'food habit': ab,ti OR 'beverage habit': ab,ti OR 'dietary profile"':ab,ti OR 'food profile*':ab,ti OR 'diet profile': $: a b, t i$ OR 'eating profile*':ab,ti OR 'dietary guideline ${ }^{* \prime}: a b, t i$ OR 'dietary recommendation"':ab,ti OR 'dietary intake ${ }^{* \prime}: a b, t i$ OR 'eating style ${ }^{*}: a b, t i$ OR 'mediterranean diet $^{* \prime}: a b$, ti OR 'dietary approaches to stop hypertension diet ${ }^{* \prime}: a b, t i$ OR 'dash diet $^{* \prime}: a b, t i$ OR 'gluten free diet $^{* \prime}: a b, t i$ OR 'prudent diet"':ab,ti OR 'paleolithic diet ${ }^{* \prime}: a b, t i$ OR

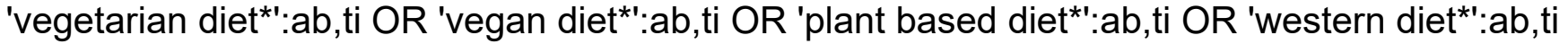
OR 'low-carbohydrate diet ${ }^{\star \prime}: a b, t i$ OR 'high carbohydrate diet"':ab,ti OR 'ketogenic diet ${ }^{\star \prime}: a b, t i$ 


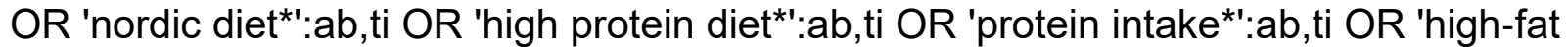
diet $^{* \prime}: a b, t i$ OR 'low fat diet*':ab,ti OR 'low protein diet*':ab,ti OR 'low-sodium diet':ab,ti OR 'low salt diet ${ }^{* \prime}: a b, t i$

\#3 - (('dietary protein*' OR 'dietary carbohydrate*' OR 'dietary fat*' OR hypocaloric OR hypocaloric) NEAR/6 (diet OR diets OR consumption OR intake OR supplement)):ab,ti

\#4 - ('guideline adherence' NEAR/6 (diet OR dietary OR food OR beverage OR nutrition*)):ab,ti

\#5 - 'diet score':ab,ti OR 'diet quality score':ab,ti OR kidmed:ab,ti OR 'diet index':ab,ti OR 'dietary index':ab,ti OR 'diet quality index':ab,ti OR 'food score':ab,ti OR meddietscore:ab,ti OR 'healthy eating index':ab,ti

\#6 - ((pattern OR patterns OR consumption OR habit*) NEAR/6 (diet OR diets OR dietary OR food OR foods OR beverage OR beverages)):ab,ti

\section{\#7 - \#1 OR \#2 OR \#3 OR \#4 OR \#5 OR \#6}

\#8 - 'sarcopenia'/de OR 'muscle atrophy'/de OR 'muscle fatigue'/de OR 'muscle strength'/de OR 'physical performance'/exp OR 'psychomotor performance'/exp

\#9 - sarcopen*:ab,ti OR presarcopen*:ab,ti OR 'muscular atroph*':ab,ti OR 'muscle fatigue $^{* \prime}: a b, t i$ OR 'muscle wast ${ }^{* \prime}: a b, t i$ OR 'muscle weak':ab,ti OR 'muscle loss ${ }^{* \prime}: a b, t i$ OR 'muscle function*':ab,ti OR 'hand strength*':ab,ti OR 'grip strength*':ab,ti OR handgrip*:ab,ti OR 'pinch strength*':ab,ti OR 'physical performance*':ab,ti OR 'psychomotor performance':ab,ti

\#10 - ((muscle OR muscles) NEAR/3 (fatigue OR weakness OR frail OR frailty OR geriatric* OR osteoporos* OR 'age factor' OR aging OR obesity OR 'body mass index' OR bmi OR 'lean mass' OR 'fat mass' OR 'daily life activity' OR 'quality of life' OR 'body equilibrium' OR posture* OR 'physical exertion*' OR 'motor activit*' OR walking OR gait)):ab,ti

\#11 - ((aging OR 'older adult*') NEAR/6 ('physical activit*' OR 'physical function' OR 'physical impairment' OR 'functional limit"' OR 'functional decline' OR 'mobility limitation*' OR walking OR 'activities of daily living' OR 'independent living' OR 'quality of life')):ab,ti

\#12 - \#8 OR \#9 OR \#10 OR \#11

\#13 - \#7 AND \#12

\#14 - \#7 AND \#12 AND ([article]/lim OR [article in press]/lim) AND [humans]/lim AND [english]/lim AND [2000-2019]/py NOT ([conference abstract]/lim OR [conference review]/lim OR [conference paper]/lim OR [editorial]/lim OR [erratum]/lim OR [letter]/lim OR [note]/lim OR [review]/lim OR [systematic review]/lim OR [meta analysis]/lim)

\section{LITERATURE SEARCH AND SCREENING RESULTS}

The flow chart (Figure 2) below illustrates the literature search and screening results for articles examining the systematic review question. The results of the electronic database searches, after removal of duplicates, were screened independently by two NESR analysts using a step-wise process by reviewing titles, abstracts, and full-texts to determine which articles met the inclusion criteria. Refer to Table $\mathbf{5}$ for the rationale for exclusion for each excluded full-text article. A manual search was done to find articles that were not identified when searching the electronic databases; all manually identified articles are also screened to determine whether they meet criteria for inclusion. 
Figure 2: Flow chart of literature search and screening results

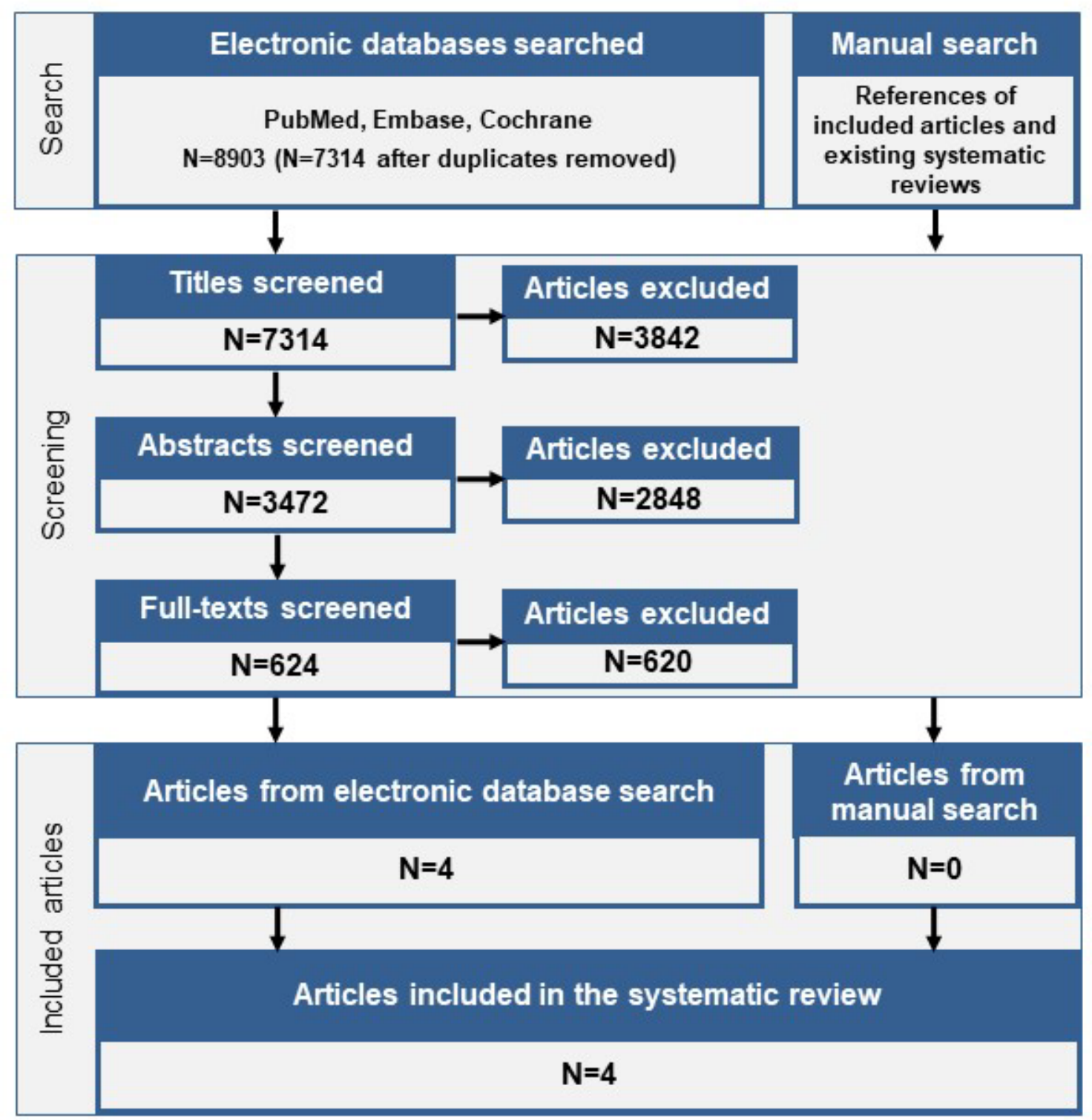




\section{Excluded Articles}

The table below lists the articles excluded after full-text screening, and includes columns for the categories of inclusion and exclusion criteria (see Table 1) that studies were excluded based on. At least one reason for exclusion is provided for each article, as indicated by an " $X$ " in one of the columns, though this may not reflect all possible reasons for exclusion. Information about articles excluded after title and abstract screening is available upon request.

\section{Table 5. Articles excluded after full text screening with rationale for exclusion}

\begin{tabular}{|c|c|c|c|c|c|c|c|c|c|}
\hline & Citation & $\begin{array}{l}\text { Study } \\
\text { Design }\end{array}$ & $\begin{array}{l}\text { Intervention } \\
\text { /Exposure }\end{array}$ & Comparator & Outcome & $\begin{array}{l}\text { Publication } \\
\text { Status }\end{array}$ & Language & Country & $\begin{array}{l}\text { Health } \\
\text { Status }\end{array}$ \\
\hline 1. & $\begin{array}{l}\text { Preserve muscle mass--and prevent falls--with proper nutrition. Protein, vitamin D, } \\
\text { and a balanced diet counter the effects of low muscle mass, promoting strength } \\
\text { and mobility as we age. Duke Med Health News. 2013. 19:3-4 } \\
\text { https://www.ncbi.nlm.nih.gov/pubmed/23802327 }\end{array}$ & $x$ & & & & $x$ & & & \\
\hline 2. & $\begin{array}{l}\text { Antoneta Granic, Carol Jagger, Karen Davies, Ashley Adamson, Thomas Kirkwood, } \\
\text { Tom R Hill, Mario Siervo, John C Mathers, Avan Aihie Sayer Effect of Dietary } \\
\text { Patterns on Muscle Strength and Physical Performance in the Very Old: Findings } \\
\text { from the Newcastle 85+ Study. PloS one. 2016. 11:e0149699 } \\
\text { https://www.ncbi.nlm.nih.gov/pubmed/26934360 }\end{array}$ & & & & $x$ & & & & \\
\hline 3. & $\begin{array}{l}\text { Abbatecola, A. M.,Cherubini, A.,Guralnik, J. M.,Andres Lacueva, C.,Ruggiero, C., } \\
\text { Maggio, M.,Bandinelli, S.,Paolisso, G.,Ferrucci, L. Plasma polyunsaturated fatty } \\
\text { acids and age-related physical performance decline. Rejuvenation Res. 2009. } \\
\text { 12:25-32 https://www.ncbi.nlm.nih.gov/pubmed/19196012 } \\
\text { https://www.ncbi.nlm.nih.gov/pmc/articles/PMC2674224/pdf/rej.2008.0799.pdf }\end{array}$ & $x$ & $x$ & & & & & & \\
\hline 4. & $\begin{array}{l}\text { Achten, J.,Halson, S. L.,Moseley, L., Rayson, M. P.,Casey, A.,Jeukendrup, A. E. } \\
\text { Higher dietary carbohydrate content during intensified running training results in } \\
\text { better maintenance of performance and mood state. J Appl Physiol (1985). } 2004 . \\
\text { 96:1331-40 https://www.ncbi.nlm.nih.gov/pubmed/14660506 } \\
\text { https://www.physiology.org/doi/pdf/10.1152/japplphysiol.00973.2003 }\end{array}$ & & & & $x$ & & & & $x$ \\
\hline 5. & $\begin{array}{l}\text { Actrn, Does nutrition and exercise prevent frailty and reduce falls in pre-frail older } \\
\text { adults in New Zealand?. Http://www.who.int/trialsearch/trial2.aspx? } \\
\text { Trialid=actrn12614000827639. 2014. \#volume\#:\#pages\# } \\
\text { https://www.cochranelibrary.com/central/doi/10.1002/central/CN-01800174/full }\end{array}$ & $x$ & & & & $x$ & & & \\
\hline 6. & $\begin{array}{l}\text { Agarwal, P.,Wang, Y.,Buchman, A. S.,Bennett, D. A.,Morris, M. C. Dietary Patterns } \\
\text { and Self-reported Incident Disability in Elderly. J Gerontol A Biol Sci Med Sci. } 2018 . \\
\text { \#volume\#:\#pages\# https://www.ncbi.nlm.nih.gov/pubmed/30247552 }\end{array}$ & & & & $x$ & & & & \\
\hline 7. & $\begin{array}{l}\text { Aguirre, N.,van Loon, L. J.,Baar, K. The role of amiX acids in skeletal muscle } \\
\text { adaptation to exercise. Nestle Nutr Inst Workshop Ser. 2013. 76:85-102 } \\
\text { https://www.ncbi.nlm.nih.gov/pubmed/23899757 } \\
\text { https://www.karger.com/Article/Abstract/350261 }\end{array}$ & $x$ & $x$ & & & & & & \\
\hline
\end{tabular}


V.,Lundbom, N.,Hakkarainen, A.,Muurinen, T.,Piirilä, P.,Pietiläinen, K. H.,

Suomalainen, A. Modified Atkins diet induces subacute selective ragged-red-fiber

lysis in mitochondrial myopathy patients. EMBO Molecular Medicine. 2016. 8:1234-

1247

http://www.embase.com/search/results?subaction=viewrecord\&from=export\&id=L6

http://www

12294551
http://dx.doi.org/10.15252/emmm.201606592

9. Ainslie, P. N.,Campbell, I. T.,Frayn, K. N.,Humphreys, S. M.,MacLaren, D. P.,Reilly,

T.,Westerterp, K. R. Energy balance, metabolism, hydration, and performance

during strenuous hill walking: the effect of age. J Appl Physiol (1985). 2002.

during strenuous hill walking: the effect of age. J App/ Physi

https://www.physiology.org/doi/pdf/10.1152/japplphysiol.01249.2001

10. Alemán-Mateo, H.,Carreón, V. R.,Macías, L.,Astiazaran-García, H.,Gallegos-

Aguilar, A. C.,Enríquez, J. R. Nutrient-rich dairy proteins improve appendicular

skeletal muscle mass and physical performance, and attenuate the loss of muscle

strength in older men and women subjects: a single-blind randomized clinical trial.

Clinical interventions in aging. 2014. 9:1517-1525

https://www.cochranelibrary.com/central/doi/10.1002/central/CN-01013922/full

11. Alexandrov, N. V.,Eelderink, C.,Singh-Povel, C. M.,Navis, G. J.,Bakker, S. J. L., Corpeleijn, E. Dietary Protein Sources and Muscle Mass over the Life Course: The Lifelines Cohort Study. Nutrients. 2018. 10:\#pages\#

https://www.ncbi.nlm.nih.gov/pubmed/30308987

https://res.mdpi.com/nutrients/nutrients-10-01471/article_deploy/nutrients-10-

01471. pdf?filename $=$ \&attachment $=1$

12. Allaert, F. A.,Guerin-Deremaux, L.,Mauray-Soulier, A.,Saniez-Degrave, M. H. Evaluation of adherence by elderly nursing home patients to regular consumption of apple compote enriched with protein and soluble fiber. Aging Clin Exp Res. 2016. 28:189-95 https://www.ncbi.nlm.nih.gov/pubmed/26240032

https://www.ncbi.nlm.nih.gov/pmc/articles/PMC4794522/pdf/40520_2015_Article_4 15.pdf

13. Alves, C. R.,Merege Filho, C. A.,Benatti, F. B.,Brucki, S.,Pereira, R. M.,de Sa Pinto, A. L.,Lima, F. R.,Roschel, H.,GualaX, B. Creatine supplementation associated or not with strength training upon emotional and cognitive measures in older women: a randomized double-blind study. PLoS One. 2013. 8:e76301

https://www.ncbi.nlm.nih.gov/pubmed/24098469

https://www.ncbi.nlm.nih.gov/pubmed/24098469
https://www.ncbi.nlm.nih.gov/pmc/articles/PMC3789718/pdf/pone.0076301.pdf

14. Alves, C. R.,Pasqua, L.,Artioli, G. G.,Roschel, H.,Solis, M.,Tobias, G.,Klansener C.,Bertuzzi, R.,Franchini, E.,Lancha Junior, A. H.,Gualano, B. Anthropometric,

physiological, performance, and nutritional profile of the Brazil National Canoe Polo Team. J Sports Sci. 2012. 30:305-11

https://www.ncbi.nlm.nih.gov/pubmed/22168392

https://www.tandfonline.com/doi/full/10.1080/02640414.2011.638086

15. Anderson, A. L.,Harris, T. B., Tylavsky, F. A.,Perry, S. E.,Houston, D. K.,Hue, T. F., Strotmeyer, E. S.,Sahyoun, N. R. Dietary patterns and survival of older adults. J Am Diet Assoc. 2011. 111:84-91 https://www.ncbi.nlm.nih.gov/pubmed/21185969 
16. Andrews, R. D.,MacLean, D. A.,Riechman, S. E. Protein intake for skeletal muscle hypertrophy with resistance training in seniors. Int J Sport Nutr Exerc Metab. 2006. 16:362-72 https://www.ncbi.nlm.nih.gov/pubmed/17136939

17. Andrich, D. E.,Filion, M. E.,Woods, M.,Dwyer, J. T., Gorbach, S. L., Goldin, B. R., Adlercreutz, H.,Aubertin-Leheudre, M. Relationship between essential amino acids and muscle mass, independent of habitual diets, in pre- and post-menopausal US women. Int J Food Sci Nutr. 2011. 62:719-24

https://www.ncbi.nlm.nih.gov/pubmed/21568818

https://www.tandfonline.com/doi/pdf/10.3109/09637486.2011.573772?needAccess =true

18. Aparicio, V. A.,Coll-Risco, I.,Borges-Cosic, M.,Acosta-Manzano, P. Exercise and the mediterranean diet for the prevention and treatment of cardiometabolic abnormalities induced by menopause. The flamenco study. Journal of physiology and biochemistry. 2018. $74:$ S12-S13

https://www.cochranelibrary.com/central/doi/10.1002/central/CN-01770599/full

19. Aparicio, V. A.,Ruiz-Cabello, P.,Borges-Cosic, M.,Andrade, A.,Coll-Risco, I.,AcostaManzano, P.,Soriano-Maldonado, A. Association of physical fitness, body composition, cardiometabolic markers and adherence to the Mediterranean diet with bone mineral density in perimenopausal women. The FLAMENCO project. Sports Sci. 2017. 35:880-887 https://www.ncbi.nlm.nih.gov/pubmed/27310097 https://www.tandfonline.com/doi/full/10.1080/02640414.2016.1196825

20. Aptekmann, N. P..Cesar, T. B. Orange juice improved lipid profile and blood lactate of overweight middle-aged women subjected to aerobic training. Maturitas. 2010 67:343-7 https://www.ncbi.nlm.nih.gov/pubmed/20729016

https://www.sciencedirect.com/science/article/pii/S0378512210003075?via\%3Dihu b

21. Arentson-Lantz, E. J Galvan, E.,Ellison, J Wacher, A Paddon-Jones, D. Improving Dietary Protein Quality Reduces the Negative Effects of Physical Inactivity on Body Composition and Muscle Function. J Gerontol A Biol Sci Med Sci. 2019. 74:16051611 https://www.ncbi.nlm.nih.gov/pubmed/30689727

22. Argyridou, S.,Zaccardi, F.,Davies, M. J.,Khunti, K.,Yates, T. Relevance of physical function in the association of red and processed meat intake with all-cause, cardiovascular, and cancer mortality. Nutr Metab Cardiovasc Dis. 2019. \#volume\#:\#pages\# https://www.ncbi.nlm.nih.gov/pubmed/31377183

23. Arias-Fernandez, L.,Machado-Fragua, M. D.,Graciani, A.,Guallar-Castillon, P., Banegas, J. R.,Rodriguez-Artalejo, F.,Lana, A.,Lopez-Garcia, E. Prospective Association Between Nut Consumption and Physical Function in Older Men and Women. J Gerontol A Biol Sci Med Sci. 2018. \#volume\#:\#pages\# https://www.ncbi.nlm.nih.gov/pubmed/30052782

https://academic.oup.com/biomedgerontology/advance-articleabstract/doi/10.1093/gerona/gly171/5056957? redirectedFrom=fulltext

24. Armamento-Villareal, R.,Aguirre, L.,Napoli, N.,Shah, K.,Hilton, T.,Sinacore, D. R. Qualls, C.,Villareal, D. T. Changes in thigh muscle volume predict bone mineral density response to lifestyle therapy in frail, obese older adults. Osteoporos Int. 2014. 25:551-8 https://www.ncbi.nlm.nih.gov/pubmed/23892583

https://www.ncbi.nlm.nih.gov/pmc/articles/PMC3903658/pdf/nihms528844.pdf 
25. Arnarson, A., Gudny Geirsdottir, O.,Ramel, A.,Briem, K.,Jonsson, P. V.,Thorsdottir, I. Effects of whey proteins and carbohydrates on the efficacy of resistance training in elderly people: Double blind, randomised controlled trial. European Journal of Clinical Nutrition. 2013. 67:821-826

http://www.embase.com/search/results?subaction=viewrecord\&from=export\&id=L5 2488267

http://dx.doi.org/10.1038/ejcn.2013.40

26. Asamane, E. A.,Greig, C. A.,Aunger, J. A.,Thompson, J. L. Perceptions and factors influencing eating behaviours and physical function in community-dwelling

ethnically diverse older adults: A longitudinal qualitative study. Nutrients. 2019.

11:\#pages\#

http://www.embase.com/search/results?subaction=viewrecord\&from=export\&id=L2 002186069

http://dx.doi.org/10.3390/nu11061224

27. Assmann, K. E.,Andreeva, V. A.,Camilleri, G. M.,Verger, E. O.,Jeandel, C., Hercberg, S., Galan, P.,Kesse-Guyot, E. Dietary scores at midlife and healthy ageing in a French prospective cohort. Br J Nutr. 2016. 116:666-76 https://www.ncbi.nlm.nih.gov/pubmed/27301412

28. Assmann, K. E.,Lassale, C.,Andreeva, V. A.,Jeandel, C.,Hercberg, S., Galan, P., Kesse-Guyot, E. A Healthy Dietary Pattern at Midlife, Combined with a Regulated Energy Intake, Is Related to Increased Odds for Healthy Aging. J Nutr. 2015 145:2139-45 https://www.ncbi.nlm.nih.gov/pubmed/26246320

29. Aubertin-Leheudre, M.,Adlercreutz, H. Relationship between animal protein intake and muscle mass index in healthy women. Br J Nutr. 2009. 102:1803-10 https://www.ncbi.nlm.nih.gov/pubmed/19678968 https://www.cambridge.org/core/services/aop-cambridge-

core/content/view/FDD4EBFB12C8089E4FF69555FF6BB98C/S000711450999131 0a.pdf/div-class-title-relationship-between-animal-protein-intake-and-muscle-massindex-in-healthy-women-div.pdf

30. Aubertin-Leheudre, M.,Lord, C.,Labonte, M.,Khalil, A.,Dionne, I. J. Relationship between sarcopenia and fracture risks in obese postmenopausal women. $J$ Women Aging. 2008. 20:297-308 https://www.ncbi.nlm.nih.gov/pubmed/18983113

31. Aubry, E.,Aeberhard, C.,Bally, L.,Nuoffer, J. M.,Risch, L.,Muhlebach, S.,Burgunder, J. M.,Stanga, Z. Are patients affected by mitochondrial disorders at nutritional risk? Nutrition. 2018. 47:56-62 https://www.ncbi.nlm.nih.gov/pubmed/29429536 https://www.sciencedirect.com/science/article/pii/S0899900717302125?via\%3Dihu b

32. Backx, E. M.,Tieland, M.,Borgonjen-van den Berg, K. J.,Claessen, P. R.,van Loon, L. J., de Groot, L. C. Protein intake and lean body mass preservation during energy intake restriction in overweight older adults. Int J Obes (Lond). 2016. 40:299-304 https://www.ncbi.nlm.nih.gov/pubmed/26471344 https://www nature com/articles/ijo2015182 pdf

33. Baguet, A.,Everaert, I.,De Naeyer, H., Reyngoudt, H.,Stegen, S.,Beeckman, S., Achten, E.,Vanhee, L.,Volkaert, A.,Petrovic, M.,Taes, Y.,Derave, W. Effects of sprint training combined with vegetarian or mixed diet on muscle carnosine content and buffering capacity. Eur J Appl Physiol. 2011. 111:2571-80

https://www.ncbi.nlm.nih.gov/pubmed/21373871

https://link.springer.com/content/pdf/10.1007\%2Fs00421-011-1877-4.pdf 
long changes in protein metabolism in elderly men and women supplemented with

a nutrition cocktail of beta-hydroxy-beta-methylbutyrate (HMB), L-arginine, and L-

lysine. JPEN J Parenter Enteral Nutr. 2009. 33:71-82

https://www.ncbi.nlm.nih.gov/pubmed/19164608

https://onlinelibrary.wiley.com/doi/pdf/10.1177/0148607108322403

35. Baker, M. E.,DeCesare, K. N.,Johnson, A.,Kress, K. S.,Inman, C. L.,Weiss, E. P Short-Term Mediterranean Diet Improves Endurance Exercise Performance: A

Randomized-Sequence Crossover Trial. J Am Coll Nutr. 2019. \#volume\#:1-9

https://www.ncbi.nlm.nih.gov/pubmed/30758261

36. Bakker, L. E.,van Schinkel, L. D.,Guigas, B.,Streefland, T. C.,Jonker, J. T.,van Klinken, J. B.,van der Zon, G. C.,Lamb, H. J.,Smit, J. W.,Pijl, H.,Meinders, A. E.,

Jazet, I. M. A 5-day high-fat, high-calorie diet impairs insulin sensitivity in healthy, young South Asian men but not in Caucasian men. Diabetes. 2014. 63:248-58 https://www.ncbi.nlm.nih.gov/pubmed/24357702

http://hw-f5-diabetes.highwire.org/content/diabetes/63/1/248.full.pdf

37. Barbat-Artigas, S.,Dupontgand, S.,Fex, A.,Karelis, A. D.,Aubertin-Leheudre, M. Relationship between dynapenia and cardiorespiratory functions in healthy postmenopausal women: novel clinical criteria. Menopause. 2011. 18:400-5 https://www.ncbi.nlm.nih.gov/pubmed/21107297

38. Barnekow-Bergkvist, M.,Hedberg, G.,Janlert, U.,Jansson, E. Adolescent determinants of cardiovascular risk factors in adult men and women. Scand $J$ Public Health. 2001. 29:208-17 https://www.ncbi.nlm.nih.gov/pubmed/11680773

39. Barrea, L.,Muscogiuri, G.,Di Somma, C., Tramontano, G.,De Luca, V.,Illario, M., Colao, A.,Savastano, S. Association between Mediterranean diet and hand grip strength in older adult women. Clin Nutr. 2019. 38:721-729

https://www.ncbi.nlm.nih.gov/pubmed/29643004

https://www.sciencedirect.com/science/article/pii/S0261561418301249?via\%3Dihu b

40. Bartali, B.,Frongillo, E. A.,Stipanuk, M. H.,Bandinelli, S.,Salvini, S.,Palli, D.,Morais, J. A.,Volpato, S.,Guralnik, J. M.,Ferrucci, L. Protein intake and muscle strength in older persons: does inflammation matter? J Am Geriatr Soc. 2012 60:480-4 https://www.ncbi.nlm.nih.gov/pubmed/22283208

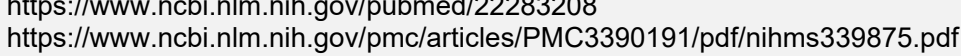

41. Baruth, M.,Wilcox, S.,McClenaghan, B.,Becofsky, K.,Schoffman, D. E. Clinically meaningful changes in functional performance resulting from self-directed interventions in individuals with arthritis. Public health. 2016. 133:116-123 https://www.cochranelibrary.com/central/doi/10.1002/central/CN-01154725/full

42. Bauer, J. M.,Verlaan, S.,Bautmans, I.,Brandt, K.,Donini, L. M.,Maggio, M. McMurdo, M. E.,Mets, T.,Seal, C.,Wijers, S. L.,Ceda, G. P.,De Vito, G.,Donders, G.,Drey, M.,Greig, C.,Holmback, U.,Narici, M.,McPhee, J.,Poggiogalle, E.,Power, D.,Scafoglieri, A.,Schultz, R.,Sieber, C. C.,Cederholm, T. Effects of a vitamin D and leucine-enriched whey protein nutritional supplement on measures of sarcopenia in older adults, the PROVIDE study: a randomized, double-blind, placebo-controlled trial. J Am Med Dir Assoc. 2015. 16:740-7

https://www.ncbi.nlm.nih.gov/pubmed/26170041 
Baumgartner, R. N. Body composition in healthy aging. Ann NY Acad Sci. 2000

$\mathrm{X}$

904:437-48 https://www.ncbi.nlm.nih.gov/pubmed/10865787

6632.2000.tb06498.x

44. Bayán-Bravo, A.,Pérez-Tasigchana, R. F.,Sayón-Orea, C.,Martínez-Gómez, D., López-García, E.,Rodríguez-Artalejo, F.,Guallar-Castillón, P. Combined impact of traditional and non-traditional healthy behaviors on health-related quality of Life: $A$ prospective study in older adults. PLoS ONE. 2017. 12:\#pages\#

http://www.embase.com/search/results?subaction=viewrecord\&from=export\&id=L6 14210164

http://dx.doi.org/10.1371/journal.pone.0170513

45. Beasley, J. M.,Lacroix, A. Z.,Neuhouser, M. L.,Huang, Y.,Tinker, L.,Woods, N.,

Michael, Y.,Curb, J. D.,Prentice, R. L. Protein intake and incident frailty in the

women's health initiative observational study. Journal of the American Geriatrics Society. 2010. 58:1063-1071

http://www.embase.com/search/results?subaction=viewrecord\&from=export\&id=L3 58945013

http://dx.doi.org/10.1111/j.1532-5415.2010.02866.x

46. Beasley, J. M.,Sevick, M. A.,Kirshner, L.,Mangold, M.,Chodosh, J. Congregate Meals: Opportunities to Help Vulnerable Older Adults Achieve Diet and Physical Activity Recommendations. J Frailty Aging. 2018. 7:182-186 https://www.ncbi.nlm.nih.gov/pubmed/30095149

47. Beasley, J. M.,Wertheim, B. C.,LaCroix, A. Z.,Prentice, R. L.,Neuhouser, M. L Tinker, L. F.,Kritchevsky, S.,Shikany, J. M.,Eaton, C.,Chen, Z., Thomson, C. A. Biomarker-calibrated protein intake and physical function in the Women's Health Initiative. J Am Geriatr Soc. 2013. 61:1863-71

https://www.ncbi.nlm.nih.gov/pubmed/24219187

https://www.ncbi.nlm.nih.gov/pmc/articles/PMC3928025/pdf/nihms554126.pdf

48. Bechshoft, R. L.,Reitelseder, S.,Hojfeldt, G.,Castro-Mejia, J. L.,Khakimov, B., Ahmad, H. F.,Kjaer, M.,Engelsen, S. B.,Johansen, S. M.,Rasmussen, M. A., Lassen, A. J.,Jensen, T.,Beyer, N.,Serena, A.,Perez-Cueto, F. J.,Nielsen, D. S., Jespersen, A. P.,Holm, L. Counteracting Age-related Loss of Skeletal Muscle Mass: a clinical and ethnological trial on the role of protein supplementation and training load (CALM Intervention Study): study protocol for a randomized controlled trial. Trials. 2016. 17:397 https://www.ncbi.nlm.nih.gov/pubmed/27507236 https://www.ncbi.nlm.nih.gov/pmc/articles/PMC4977774/pdf/13063_2016_Article_1 512.pdf

49. Beck, A. M.,Damkjaer, K.,Beyer, N. Multifaceted nutritional intervention among nursing-home residents has a positive influence on nutrition and function. Nutrition. 2008. 24:1073-80 https://www.ncbi.nlm.nih.gov/pubmed/18586458

https://www.sciencedirect.com/science/article/pii/S0899900708002189?via\%3Dihu b

50. Beck, A. M.,Rask, KØ,Leedo, E.,Jensen, L. L.,Martins, K.,Vedelspang, A Nutritional support in a cross-sector model for the rehabilitation of geriatric patients: a randomised controlled trial. Clinical nutrition (edinburgh, scotland). 2014. 33:S37https://www.cochranelibrary.com/central/doi/10.1002/central/CN-01053089/full

(1)


51. Beelen, J.,de Roos, N. M.,de Groot, Lcpgm A 12-week intervention with protein-

$\mathrm{X}$

enriched foods and drinks improved protein intake but not physical performance of

older patients during the first 6 months after hospital release: a randomised

controlled trial. Br J Nutr. 2017. 117:1541-1549

https://www.ncbi.nlm.nih.gov/pubmed/28662731

https://www.cambridge.org/core/services/aop-cambridge-

core/content/view/183D909E5E48458A1C4FF0411B364101/S0007114517001477

a.pdf/div-class-title-a-12-week-intervention-with-protein-enriched-foods-and-drinks-

improved-protein-intake-but-not-physical-performance-of-older-patients-during-the-

first-6-months-after-hospital-release-a-randomised-controlled-trial-div.pdf

52. Bell, K. E.,Snijders, T.,Zulyniak, M.,Kumbhare, D.,Parise, G.,Chabowski, A.,Phillips, S. M. A whey protein-based multi-ingredient nutritional supplement stimulates gains in lean body mass and strength in healthy older men: A randomized controlled trial. PLoS One. 2017. 12:e0181387 https://www.ncbi.nlm.nih.gov/pubmed/28719669 https://www.ncbi.nlm.nih.gov/pmc/articles/PMC5515445/pdf/pone.0181387.pdf

53. Beller, G.,Belavy, D. L.,Sun, L.,Armbrecht, G.,Alexandre, C.,Felsenberg, D. WISE2005: bed-rest induced changes in bone mineral density in women during 60 days simulated microgravity. Bone. 2011. 49:858-66

https://www.ncbi.nlm.nih.gov/pubmed/21723970

https://www.sciencedirect.com/science/article/pii/S8756328211010623?via\%3Dihu b

54. Berg, A.,Frey, I.,Deibert, P.,Landmann, U.,Konig, D.,Schmidt-Trucksass, A.,Rucker, G.,Kreiter, H.,Berg, A.,Dickhuth, H. H. Weight reduction is feasible - Preliminary results of a controlled, randomised intervention study in overweight adults.

Ernahrungs umschau. 2003. 50:386-393+374

https://www.cochranelibrary.com/central/doi/10.1002/central/CN-01754472/full

55. Bernstein, M. A., Tucker, K. L.,Ryan, N. D.,O'Neill, E. F.,Clements, K. M.,Nelson, M. E.,Evans, W. J.,Fiatarone Singh, M. A. Higher dietary variety is associated with better nutritional status in frail elderly people. J Am Diet Assoc. 2002. 102:1096-104 https://www.ncbi.nlm.nih.gov/pubmed/12171454

56. Berryman, C. E.,Sepowitz, J. J.,McClung, H. L.,Lieberman, H. R.,Farina, E. K., McClung, J. P.,Ferrando, A. A.,Pasiakos, S. M. Supplementing an energy adequate, higher protein diet with protein does not enhance fat-free mass restoration after short-term severe negative energy balance. J Appl Physiol (1985). 2017. 122:1485-1493 https://www.ncbi.nlm.nih.gov/pubmed/28385919

57. Bertoli, S.,Magni, P.,Krogh, V.,Ruscica, M.,Dozio, E.,Testolin, G.,Battezzati, A. Is ghrelin a signal of decreased fat-free mass in elderly subjects?. Eur J Endocrinol. 2006. 155:321-30 https://www.ncbi.nlm.nih.gov/pubmed/16868147

58. Bezrati-Benayed, I.,Nasrallah, F.,Feki, M.,Chamari, K.,Omar, S.,Alouane-Trabelsi, L.,Ben Mansour, A.,Kaabachi, N. Urinary creatine at rest and after repeated sprints in athletes: a pilot study. Biol Sport. 2014. 31:49-54

https://www.ncbi.nlm.nih.gov/pubmed/24917689 
McCarthy, A. C.,Woodbury, E.,McKinnon, J.,Fleck, G.,Storer, T. W.,Basaria, S.

Effect of Protein Intake on Lean Body Mass in Functionally Limited Older Men: A

Randomized Clinical Trial. JAMA Intern Med. 2018. 178:530-541

https://www.ncbi.nlm.nih.gov/pubmed/29532075

60. Bibas, L.,Levi, M.,Bendayan, M.,Mullie, L.,Forman, D. E.,Afilalo, J. Therapeutic

interventions for frail elderly patients: Part I: Published randomized trials. Progress in Cardiovascular Diseases. 2014. 57:134-143

http://www.embase.com/search/results?subaction=viewrecord\&from=export\&id=L6 04626599

http://dx.doi.org/10.1016/j.pcad.2014.07.004

61. Bilsborough, J. C.,Greenway, K.,Livingston, S.,Cordy, J.,Coutts, A. J. Changes in

Anthropometry, Upper-Body Strength, and Nutrient Intake in Professional

Australian Football Players During a Season. International journal of sports

physiology and performance. 2016. 11:290-300

http://www.embase.com/search/results?subaction=viewrecord\&from=export\&id=L6

15156012

http://dx.doi.org/10.1123/ijspp.2014-0447

62. Bingham, C. M.,Lahti-Koski, M.,Absetz, P.,Puukka, P.,Kinnunen, M.,Pihlajamaki, H.,Sahi, T.,Uutela, A.,Jallinoja, P. Food choices and health during military service: increases in sugar- and fibre-containing foods and changes in anthropometric and clinical risk factors. Public Health Nutr. 2012. 15:1248-55

https://www.ncbi.nlm.nih.gov/pubmed/22166515

63. Binns, A.,Gray, M.,Henson, A. C.,Fort, I. L. Changes in Lean Mass and Serum Myostatin with Habitual Protein Intake and High-Velocity Resistance Training. J Nutr Health Aging. 2017. 21:1111-1117

https://www.ncbi.nlm.nih.gov/pubmed/29188869

64. Birnie, K.,Ben-Shlomo, Y., Gunnell, D.,Ebrahim, S.,Bayer, A., Gallacher, J.,Holly, J. M.,Martin, R. M. Childhood milk consumption is associated with better physical performance in old age. Age Ageing. 2012. 41:776-84

https://www.ncbi.nlm.nih.gov/pubmed/22542496

65. Bischoff-Ferrari, H. A.,Dietrich, T.,Orav, E. J.,Hu, F. B.,Zhang, Y.,Karlson, E. W., Dawson-Hughes, B. Higher 25-hydroxyvitamin D concentrations are associated with better lower-extremity function in both active and inactive persons aged $>$ or $=60$ y. Am J Clin Nutr. 2004. 80:752-8

https://www.ncbi.nlm.nih.gov/pubmed/15321818

66. Bishop, N. J.,Zuniga, K. E.,Lucht, A. L. Latent Profiles of Macronutrient Density and their Association with Mobility Limitations in an Observational Longitudinal Study of Older U.S. Adults. J Nutr Health Aging. 2018. 22:645-654

https://www.ncbi.nlm.nih.gov/pubmed/29806853

67. Blundell, J. E.,Caudwell, P.,Gibbons, C.,Hopkins, M.,Naslund, E.,King, N. A.,

Finlayson, G. Body composition and appetite: fat-free mass (but not fat mass or

$\mathrm{BMI}$ ) is positively associated with self-determined meal size and daily energy intake

in humans. Br J Nutr. 2012. 107:445-9

https://www.ncbi.nlm.nih.gov/pubmed/21733267 


$\mathrm{X}$

69. Boidin, M.,Lapierre, G.,Paquette Tanir, L.,Nigam, A.,Juneau, M.,Guilbeault, V. Latour, E.,Gayda, M. Effect of aquatic interval training with Mediterranean diet counseling in obese patients: results of a preliminary study. Ann Phys Rehabil Med. 2015. 58:269-75 https://www.ncbi.nlm.nih.gov/pubmed/26233941

70. Bollwein, J.,Diekmann, R.,Kaiser, M. J.,Bauer, J. M.,Uter, W.,Sieber, C. C.,Volkert, D. Distribution but not amount of protein intake is associated with frailty: A crosssectional investigation in the region of Nürnberg. Nutrition Journal. 2013.

12:\#pages\#

http://www.embase.com/search/results?subaction=viewrecord\&from=export\&id=L5 2720716

http://dx.doi.org/10.1186/1475-2891-12-109

71. Bopp, M. J.,Houston, D. K.,Lenchik, L.,Easter, L.,Kritchevsky, S. B.,Nicklas, B. J. Lean mass loss is associated with low protein intake during dietary-induced weight loss in postmenopausal women. J Am Diet Assoc. 2008. 108:1216-20 https://www.ncbi.nlm.nih.gov/pubmed/18589032

72. Bos, C.,Benamouzig, R.,Bruhat, A.,Roux, C., Valensi, P.,,Ferriere, F.,Tome, D. Nutritional status after short-term dietary supplementation in hospitalized malnourished geriatric patients. Clin Nutr. 2001. 20:225-33

https://www.ncbi.nlm.nih.gov/pubmed/11407869

73. Bossingham, M. J.,Carnell, N. S.,Campbell, W. W. Water balance, hydration status, and fat-free mass hydration in younger and older adults. Am J Clin Nutr. 2005. 81:1342-50 https://www.ncbi.nlm.nih.gov/pubmed/15941885

74. Bouchard, D. R.,Heroux, M.,Janssen, I. Association between muscle mass, leg strength, and fat mass with physical function in older adults: influence of age and sex. J Aging Health. 2011. 23:313-28 https://www.ncbi.nlm.nih.gov/pubmed/21081704

75. Bouillanne, O.,Curis, E.,Hamon-Vilcot, B.,Nicolis, I.,Chretien, P.,Schauer, N Vincent, J. P.,Cynober, L.,Aussel, C. Impact of protein pulse feeding on lean mass in malnourished and at-risk hospitalized elderly patients: a randomized controlled trial. Clin Nutr. 2013. 32:186-92 https://www.ncbi.nlm.nih.gov/pubmed/22992307

76. Bradlee, M. L.,Mustafa, J.,Singer, M. R.,Moore, L. L. High-Protein Foods and Physical Activity Protect Against Age-Related Muscle Loss and Functional Decline. J Gerontol A Biol Sci Med Sci. 2017. 73:88-94 https://www.ncbi.nlm.nih.gov/pubmed/28549098

77. Bradley, W. J.,Cavanagh, B. P.,Douglas, W.,Donovan, T. F.,Morton, J. P.,Close, G. L. Quantification of training load, energy intake, and physiological adaptations during a rugby preseason: a case study from an elite European rugby union squad. $J$ Strength Cond Res. 2015. 29:534-44 https://www.ncbi.nlm.nih.gov/pubmed/25029003

78. Bray, G. A.,Redman, L. M.,de Jonge, L.,Covington, J.,Rood, J.,Brock, C.,Mancuso, S.,Martin, C. K.,Smith, S. R. Effect of protein overfeeding on energy expenditure measured in a metabolic chamber. Am J Clin Nutr. 2015. 101:496-505 https://www.ncbi.nlm.nih.gov/pubmed/25733634 
80. Brown, J. C.,Harhay, M. O.,Harhay, M. N. Sarcopenia and mortality among a population-based sample of community-dwelling older adults. Journal of Cachexia, Sarcopenia and Muscle. 2015. \#volume\#:\#pages\#

http://www.embase.com/search/results?subaction=viewrecord\&from=export\&id=L6

07444252

http://dx.doi.org/10.1002/jcsm.12073

81. Brown, J. C.,Harhay, M. O.,Harhay, M. N. Physical Activity, Diet Quality, and Mortality among Community-Dwelling Prefrail and Frail Older Adults. J Nutr Gerontol Geriatr. 2016. 35:253-266

https://www.ncbi.nlm.nih.gov/pubmed/27897612

82. Buch, A.,Eldor, R.,Kis, O.,Keinan-Boker, L.,Dunsky, A.,Rubin, A.,Lopez, A.,Sofer, Y.,Osher, E.,Marcus, Y.,Stern, N. The effect of circuit resistance training,

empagliflozin or "vegeterranean diet" on physical and metabolic function in older

subjects with type 2 diabetes: a study protocol for a randomized control trial (CEV-

65 trial). BMC Geriatr. 2019. 19:228

https://www.ncbi.nlm.nih.gov/pubmed/31438863

83. Buckley, C. M.,Austin, S.,Corfe, B. M.,Green, M. A.,Johnstone, A. M.,Stevenson, E. J.,Williams, E. A.,Brunstrom, J. M. Protein Valuation in Food Choice Is Positively Associated with Lean Mass in Older Adults. J Nutr. 2019. \#volume\#:\#pages\# https://www.ncbi.nlm.nih.gov/pubmed/31198945

84. Budui, S.,Bigolin, F., Giordano, F.,Leoni, S.,Berteotti, M.,Sartori, E.,Franceschini, L., Taddei, M.,Salvetti, S.,Castiglioni, F., Gilli, F.,Skafidas, S.,Schena, F.,Letizia

Petroni, M.,Busetto, L. Effects of an intensive inpatient rehabilitation program in elderly patients with obesity. Obesity Facts. 2019. 12:199-210

http://www.embase.com/search/results?subaction=viewrecord\&from=export\&id=L6 27137592

http://dx.doi.org/10.1159/000497461

85. Burgin, J.,Nichols, S.,Dalrymple, N. The nutritional status of clinic attendees living with HIVIAIDS in St Vincent and the Grenadines. West Indian Med J. 2008. 57:43843 https://www.ncbi.nlm.nih.gov/pubmed/19565972

86. Burke, L. M.,Hawley, J. A.,Angus, D. J.,Cox, G. R.,Clark, S. A.,Cummings, N. K. Desbrow, B.,Hargreaves, M. Adaptations to short-term high-fat diet persist during exercise despite high carbohydrate availability. Med Sci Sports Exerc. 2002. 34:8391 https://www.ncbi.nlm.nih.gov/pubmed/11782652

87. Byam-Williams, J.,Salyer, J. Factors influencing the health-related lifestyle of community-dwelling older adults. Home Healthc Nurse. 2010. 28:115-21 https://www.ncbi.nlm.nih.gov/pubmed/20147806

88. Callen, B. L. Nutritional screening in community dwelling older adults. Int J Older People Nurs. 2011. 6:272-81 https://www.ncbi.nlm.nih.gov/pubmed/21631876

Calvani, R.,Martone, A. M.,Marzetti, E.,Onder, G.,Savera, G.,Lorenzi, M.,Serafini, E.,Bernabei, R.,Landi, F. Pre-hospital dietary intake correlates with muscle mass at the time of fracture in older hip-fractured patients. Front Aging Neurosci. 2014.

6:269 https://www.ncbi.nlm.nih.gov/pubmed/25477815 
Campbell, B. I.,Aguilar, D.,Conlin, L.,Vargas, A.,Schoenfeld, B. J.,Corson, A.,Gai, C.,Best, S., Galvan, E.,Couvillion, K. Effects of High Versus Low Protein Intake on Body Composition and Maximal Strength in Aspiring Female Physique Athletes

Engaging in an 8-Week Resistance Training Program. Int J Sport Nutr Exerc

Metab. 2018. 28:580-585 https://www.ncbi.nlm.nih.gov/pubmed/29405780

91. Campbell, W. W.,Trappe, T. A.,Jozsi, A. C.,Kruskall, L. J.,Wolfe, R. R.,Evans, W. J. Dietary protein adequacy and lower body versus whole body resistive training in older humans. J Physiol. 2002. 542:631-42

https://www.ncbi.nlm.nih.gov/pubmed/12122158

92. Campbell, W. W., Trappe, T. A.,Wolfe, R. R.,Evans, W. J. The recommended dietary allowance for protein may not be adequate for older people to maintain skeletal muscle. J Gerontol A Biol Sci Med Sci. 2001. 56:M373-80

https://www.ncbi.nlm.nih.gov/pubmed/11382798

93. Cardon-Thomas, D. K., Riviere, T.,Tieges, Z., Greig, C. A. Dietary Protein in Older Adults: Adequate Daily Intake but Potential for Improved Distribution. Nutrients. 2017. 9:\#pages\# https://www.ncbi.nlm.nih.gov/pubmed/28241469

94. Cardoso, G. A.,Salgado, J. M.,Cesar Mde, C.,Donado-Pestana, C. M. The effects of green tea consumption and resistance training on body composition and resting metabolic rate in overweight or obese women. J Med Food. 2013. 16:120-7 https://www.ncbi.nlm.nih.gov/pubmed/23140132

95. Carru, C.,Da Boit, M.,Paliogiannis, P.,Zinellu, A.,Sotgia, S.,Sibson, R.,Meakin, J. R.,Aspden, R. M.,Mangoni, A. A., Gray, S. R. Markers of oxidative stress, skeletal muscle mass and function, and their responses to resistance exercise training in older adults. Experimental gerontology. 2018. 103:101-106

https://www.cochranelibrary.com/central/doi/10.1002/central/CN-01461497/full

96. Carty, C. L.,Kooperberg, C.,Neuhouser, M. L., Tinker, L.,Howard, B.,WactawskiWende, J.,Beresford, S. A.,Snetselaar, L., Vitolins, M.,Allison, M.,Budrys, N., Prentice, R.,Peters, U. Low-fat dietary pattern and change in body-composition traits in the Women's Health Initiative Dietary Modification Trial. Am J Clin Nutr. 2011. 93:516-24 https://www.ncbi.nlm.nih.gov/pubmed/21177798

97. Castaneda, C., Gordon, P. L.,Fielding, R. A.,Evans, W. J.,Crim, M. C. Marginal protein intake results in reduced plasma IGF-I levels and skeletal muscle fiber atrophy in elderly women. $J$ Nutr Health Aging. 2000. 4:85-90 https://www.ncbi.nlm.nih.gov/pubmed/10842420

98. Castaneda, C.,Janssen, I. Ethnic comparisons of sarcopenia and obesity in diabetes. Ethn Dis. 2005. 15:664-70 https://www.ncbi.nlm.nih.gov/pubmed/16259491

99. Catapano, G.,Pedone, C.,Nunziata, E.,Zizzo, A.,Passantino, A.,Incalzi, R. A. Nutrient intake and serum cytokine pattern in elderly people with heart failure. Eur $J$ Heart Fail. 2008. 10:428-34 https://www.ncbi.nlm.nih.gov/pubmed/18353717

100. Cervo, M. M. Shivappa, N.,Hebert, J. R. Oddy, W. H., Winzenberg, T.,Balogun, S., Wu, F.,Ebeling, P.,Aitken, D.,Jones, G.,Scott, D. Longitudinal associations between dietary inflammatory index and musculoskeletal health in community-dwelling older adults. Clin Nutr. 2019. \#volume\#:\#pages\# https://www.ncbi.nlm.nih.gov/pubmed/30852031

$\mathrm{X}$

$\mathrm{X}$

$\mathrm{X}$

$\mathrm{X}$

.

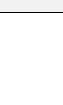

$x$

$\mathrm{X}$

X

$\mathrm{x} \quad \mathrm{x} \quad \mathrm{x}$

$\mathrm{X}$

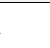

$\mathrm{X}$

$\mathrm{X}$



Atkinson, H.,Martin, A.,Guralnik, J. M.,Ferrucci, L. Antioxidants and physica performance in elderly persons: the Invecchiare in Chianti (InCHIANTI) study. Am J Clin Nutr. 2004. 79:289-94 https://www.ncbi.nlm.nih.gov/pubmed/14749236

102. Chan, R.,Leung, J.,Woo, J.,Kwok, T. Associations of dietary protein intake on subsequent decline in muscle mass and physical functions over four years in ambulant older Chinese people. J Nutr Health Aging. 2014. 18:171-7 https://www.ncbi.nlm.nih.gov/pubmed/24522470

103. Chaput, J. P.,Lord, C.,Cloutier, M.,Aubertin Leheudre, M.,Goulet, E. D.,Rousseau, S.,Khalil, A.,Dionne, I. J. Relationship between antioxidant intakes and class I sarcopenia in elderly men and women. J Nutr Health Aging. 2007. 11:363-9 https://www.ncbi.nlm.nih.gov/pubmed/17653501

104. Charlton, K.,Walton, K.,Batterham, M.,Brock, E.,Langford, K.,McMahon, A., Roodenrys, S.,Koh, F.,Host, A.,Crowe, R.,Thornhill, K. Pork and Chicken Meals Similarly Impact on Cognitive Function and Strength in Community-Living Older Adults: A Pilot Study. J Nutr Gerontol Geriatr. 2016. 35:124-45 https://www.ncbi.nlm.nih.gov/pubmed/27153252

105. Chevalier, S.,Gougeon, R.,Nayar, K.,Morais, J. A. Frailty amplifies the effects of aging on protein metabolism: role of protein intake. Am J Clin Nutr. 2003. 78:422-9 https://www.ncbi.nlm.nih.gov/pubmed/12936924

106. Chiang, P. H.,Wahlqvist, M. L.,Huang, L. Y.,Chang, Y. C. Leisure time physical activities and dietary quality of the general and indigenous Taiwanese population are associated with fat distribution and sarcopenia. Asia Pac J Clin Nutr. 2013. 22:599-613 https://www.ncbi.nlm.nih.gov/pubmed/24231021

107. Chin, A. Paw M. J.,de Jong, N.,Schouten, E. G.,Hiddink, G. J.,Kok, F. J. Physical exercise and/or enriched foods for functional improvement in frail, independently living elderly: a randomized controlled trial. Arch Phys Med Rehabil. 2001. 82:811-7 https://www.ncbi.nlm.nih.gov/pubmed/11387588

108. Choi, H. R.,Kim, J.,Lim, H.,Park, Y. K. Two-Week Exclusive Supplementation of Modified Ketogenic Nutrition Drink Reserves Lean Body Mass and Improves Blood Lipid Profile in Obese Adults: A Randomized Clinical Trial. Nutrients. 2018. 10:\#pages\# https://www.ncbi.nlm.nih.gov/pubmed/30513970

109. Chomentowski, P.,Dube, J. J.,Amati, F.,Stefanovic-Racic, M.,Zhu, S.,Toledo, F. G. Goodpaster, B. H. Moderate exercise attenuates the loss of skeletal muscle mass that occurs with intentional caloric restriction-induced weight loss in older overweight to obese adults. J Gerontol A Biol Sci Med Sci. 2009. 64:575-80 https://www.ncbi.nlm.nih.gov/pubmed/19276190

110. Chun, B. O.,Yun, M. E..Kim, I. M..Lee, J. K. Effects of short-term training camp including aerobic exercise and vegetarian diet on body composition, physical fitness and blood biochemical parameters in collegians. Gazzetta Medica Italiana Archivio per le Scienze Mediche. 2017. 176:289-298 http://www.embase.com/search/results?subaction=viewrecord\&from=export\&id=L6 15773809 http://dx.doi.org/10.23736/S0393-3660.16.03277-0

111. Clamp, L. D.,Hume, D. J.,Lambert, E. V.,Kroff, J. Successful and unsuccessful weight-loss maintainers: strategies to counteract metabolic compensation following weight loss. J Nutr Sci. 2018. 7:e20

https://www.ncbi.nlm.nih.gov/pubmed/29988905 
112. Cliffe, M.,Bloodworth, L. L.,Jibani, M. M. Can malnutrition in predialysis patients be prevented by dietetic intervention?. J Ren Nutr. 2001. 11:161-5 https://www.ncbi.nlm.nih.gov/pubmed/11466667

113. Coelho-Junior, H. J.,Calvani, R.,Goncalves, I. O.,Rodrigues, B.,Picca, A.,Landi, F. Bernabei, R.,Uchida, M. C.,Marzetti, E. High relative consumption of vegetable protein is associated with faster walking speed in well-functioning older adults. Aging Clin Exp Res. 2019. \#volume\#:\#pages\# https://www.ncbi.nlm.nih.gov/pubmed/31115875

114. Coin, A.,Perissinotto, E.,Enzi, G.,Zamboni, M.,Inelmen, E. M.,Frigo, A. C.,Manzato E.,Busetto, L.,Buja, A.,Sergi, G. Predictors of low bone mineral density in the elderly: the role of dietary intake, nutritional status and sarcopenia. Eur J Clin Nutr. 2008. 62:802-9 https://www.ncbi.nlm.nih.gov/pubmed/17637603

115. Coin, A.,Sergi, G.,Beninca, P.,Lupoli, L.,Cinti, G.,Ferrara, L.,Benedetti, G.,Tomasi, G.,Pisent, C.,Enzi, G. Bone mineral density and body composition in underweight and normal elderly subjects. Osteoporos Int. 2000. 11:1043-50 https://www.ncbi.nlm.nih.gov/pubmed/11256896

116. Coker, R. H.,Miller, S.,Schutzler, S.,Deutz, N.,Wolfe, R. R. Whey protein and essential amino acids promote the reduction of adipose tissue and increased muscle protein synthesis during caloric restriction-induced weight loss in elderly, obese individuals. Nutrition journal. 2012. 11:\#pages\#

https://www.cochranelibrary.com/central/doi/10.1002/central/CN-00912677/ful

117. Colica, C.,Merra, G., Gasbarrini, A.,De Lorenzo, A.,Cioccoloni, G.,Gualtieri, P., Perrone, M. A.,Bernardini, S.,Bernardo, V.,Di Renzo, L.,Marchetti, M. Efficacy and safety of very-low-calorie ketogenic diet: a double blind randomized crossover study. Eur Rev Med Pharmacol Sci. 2017. 21:2274-2289

https://www.ncbi.nlm.nih.gov/pubmed/28537652

118. Confortin, S. C.,Ono, L. M.,Barbosa, A. R.,d'Orsi, E. Sarcopenia and its association with changes in socioeconomic, behavioral, and health factors: the EpiFloripa Elderly Study. Cadernos de saude publica. 2018. 34:e00164917

http://www.embase.com/search/results?subaction=viewrecord\&from=export\&id=L6 26975474

http://dx.doi.org/10.1590/0102-311X00164917

119. Cooke, M. B.,Brabham, B.,Buford, T. W.,Shelmadine, B. D.,McPheeters, M., Hudson, G. M.,Stathis, C.,Greenwood, M.,Kreider, R.,Willoughby, D. S. Creatine supplementation post-exercise does not enhance training-induced adaptations in middle to older aged males. Eur J Appl Physiol. 2014 114:1321-32

https://www.ncbi.nlm.nih.gov/pubmed/24633488

120. Coulson, C. E.,Williams, L. J.,Brennan, S. L.,Berk, M.,Kotowicz, M. A.,Lubman, D. Pasco, J. A. Alcohol consumption and body composition in a population-based sample of elderly Australian men. Aging Clin Exp Res. 2013. 25:183-92 https://www.ncbi.nlm.nih.gov/pubmed/23739904

121. Cramer, J. T.,Cruz-Jentoft, A. J.,Landi, F.,Hickson, M.,Zamboni, M.,Pereira, S. L., Hustead, D. S. Mustad, V. A. Impacts of High-Protein Oral Nutritional Supplements Among Malnourished Men and Women with Sarcopenia: A Multicenter,

Randomized, Double-Blinded, Controlled Trial. J Am Med Dir Assoc. 2016.

17:1044-1055 https://www.ncbi.nlm.nih.gov/pubmed/27780567

$\mathrm{X}$

$x$

X $x$

$\mathrm{X} x$

$\mathrm{x}$

$x$


Cresci, B.,Pala, L.,Poggiali, R., Guarnieri, C.,Mannucci, E.,Bigiarini, M.,Rotella, C.

$\mathrm{X}$

$\mathrm{X}$

M. False and true pre-treatment predictors of weight loss in obese pat
a program for lifestyle change. Eat Weight Disord. 2014. 19:489-94

https://www.ncbi.nlm.nih.gov/pubmed/24816471

123. Crown, A. L.,Cottle, K.,Lightman, S. L.,Falk, S.,Mohamed-Ali, V.,Armstrong, L., Millar, A. B.,Holly, J. M. What is the role of the insulin-like growth factor system in the pathophysiology of cancer cachexia, and how is it regulated?. Clin Endocrinol (Oxf). 2002. 56:723-33 https://www.ncbi.nlm.nih.gov/pubmed/12072041

124. Cupisti, A.,Licitra, R.,Chisari, C.,Stampacchia, G.,D'Alessandro, C., Galetta, F., Rossi, B.,Barsotti, G. Skeletal muscle and nutritional assessment in chronic renal failure patients on a protein-restricted diet. J Intern Med. 2004. 255:115-24 https://www.ncbi.nlm.nih.gov/pubmed/14687247

125. D'Angelo, E.,Di Blasio, A.,Di Donato, F.,Di Gregorio, S.,Di Renzo, D.,Ripari, P. Relationships between physical exercise practice, dietary behaviour and body composition in female university students. J Sports Med Phys Fitness. 2010. 50:311-7 https://www.ncbi.nlm.nih.gov/pubmed/20842092

126. da Silva, A. P.,Valente, A.,Chaves, C.,Matos, A.,Gil, A.,Santos, A. C.,Gorjao-Clara J. P.,Bicho, M. Characterization of Portuguese Centenarian Eating Habits, Nutritional Biomarkers, and Cardiovascular Risk: A Case Control Study. Oxid Med Cell Longev. 2018. 2018:5296168 https://www.ncbi.nlm.nih.gov/pubmed/29725498

127. Daly, R. M.,O'Connell, S. L.,Mundell, N. L.,Grimes, C. A.,Dunstan, D. W.,Nowson, C. A. Protein-enriched diet, with the use of lean red meat, combined with progressive resistance training enhances lean tissue mass and muscle strength and reduces circulating IL-6 concentrations in elderly women: A cluster randomized controlled trial. American Journal of Clinical Nutrition. 2014. 99:899-910

http://www.embase.com/search/results?subaction=viewrecord\&from=export\&id=L3 72703709

http://dx.doi.org/10.3945/ajcn.113.064154

128. Dalzill, C.,Nigam, A.,Juneau, M.,Guilbeault, V.,Latour, E.,Mauriege, P.,Gayda, M. Intensive lifestyle intervention improves cardiometabolic and exercise parameters in metabolically healthy obese and metabolically unhealthy obese individuals. Can J Cardiol. 2014. 30:434-40 https://www.ncbi.nlm.nih.gov/pubmed/24561010

129. Dandanell, S.,Skovborg, C.,Praest, C. B.,Kristensen, K. B.,Nielsen, M. G.,Lionett, S.,Jorgensen, S. D.,Vigelso, A.,Dela, F.,Helge, J. W. Maintaining a clinical weight loss after intensive lifestyle intervention is the key to cardiometabolic health. Obes Res Clin Pract. 2017. 11:489-498 https://www.ncbi.nlm.nih.gov/pubmed/27720417

130. Daskalopoulou, C.,Koukounari, A.,Ayuso-Mateos, J. L.,Prince, M.,Prina, A. M. Associations of Lifestyle Behaviour and Healthy Ageing in Five Latin American and the Caribbean Countries-A 10/66 Population-Based Cohort Study. Nutrients. 2018. 10:\#pages\# https://www.ncbi.nlm.nih.gov/pubmed/30380734

131. De Hoogh, I.,Doets, E.,Holthuysen, N.,Wopereis, S.,Verain, M.,Boorsma, A. Bouwman, E.,Pasman, W.,Van Erk, M.,Reinders, M. Beneficial effect of personalised lifestyle advice as compared to generic advice on wellbeing among active dutch seniors-a pilot study. Annals of nutrition \& metabolism. 2017. 71:456$457 \mathrm{https}: / /$ www.cochranelibrary.com/central/doi/10.1002/central/CN-01428951/full

132. de Luis, D. A.,Aller, R., Izaola, O., Gonzalez Sagrado, M.,Conde, R. Resting energy expenditure, cardiovascular risk factors and insulin resistance in obese patients. Ann Nutr Metab. 2005. 49:381-5 https://www.ncbi.nlm.nih.gov/pubmed/16219989

\section{$\mathrm{X}$}

X

$\mathrm{X}$
X 
de Oliveira, E. P.,McLellan, K. C.,Vaz de Arruda Silveira, L.,Burini, R. C. Dietary

$\mathrm{X}$
https://www.ncbi.nlm.nih.gov/pubmed/22417631

134. de Oliveira, E. P.,Moreto, F.,Silveira, L. V.,Burini, R. C. Dietary, anthropometric, $\quad X$ and biochemical determinants of uric acid in free-living adults. Nutr J. 2013. 12:11 https://www.ncbi.nlm.nih.gov/pubmed/23311699

135. de Souza Genaro, P.,de Medeiros Pinheiro, M.,Szejnfeld, V. L.,Martini, L. A Secondary hyperparathyroidism and its relationship with sarcopenia in elderly women. Arch Gerontol Geriatr. 2015. 60:349-53 https://www.ncbi.nlm.nih.gov/pubmed/25614177

136. Dehail, P.,Joseph, P. A.,Faux, P.,Rainfray, M.,Emeriau, J. P.,Barat, M.,BourdelMarchasson, I. Early changes in isokinetic lower limb muscle strength in recovering geriatric subjects on the basis of nutritional status. J Nutr Health Aging. 2005. 9:356-63 https://www.ncbi.nlm.nih.gov/pubmed/16222403

137. Deibert, P.,Konig, D.,Schmidt-Trucksaess, A.,Zaenker, K. S.,Frey, I.,Landmann, U., Berg, A. Weight loss without losing muscle mass in pre-obese and obese subjects induced by a high-soy-protein diet. Int J Obes Relat Metab Disord. 2004. 28:134952 https://www.ncbi.nlm.nih.gov/pubmed/15303108

138. Demling, R. H.,DeSanti, L. Effect of a hypocaloric diet, increased protein intake and resistance training on lean mass gains and fat mass loss in overweight police resistance training on lean mass gains and
officers. Ann Nutr Metab. 2000. 44:21-9

https://www.ncbi.nlm.nih.gov/pubmed/10838463

139. Denissen, K. F.,Janssen, L. M.,Eussen, S. J.,van Dongen, M. C.,Wijckmans, N. E., van Deurse, N. D.,Dagnelie, P. C. Delivery of Nutritious Meals to Elderly Receiving Home Care: Feasibility and Effectiveness. J Nutr Health Aging. 2017. 21:370-380 https://www.ncbi.nlm.nih.gov/pubmed/28346563

140. Deriemaeker, P.,Aerenhouts, D.,De Ridder, D.,Hebbelinck, M.,Clarys, P. Health aspects, nutrition and physical characteristics in matched samples of institutionalized vegetarian and non-vegetarian elderly (>65yrs). Nutr Metab (Lond). 2011. 8:37 https://www.ncbi.nlm.nih.gov/pubmed/21672249

141. Deutz, N. E. P.,Thaden, J. J.,Ten Have, G. A. M.,Walker, D. K.,Engelen, Mpkj Metabolic phenotyping using kinetic measurements in young and older healthy adults. Metabolism. 2018. 78:167-178

https://www.ncbi.nlm.nih.gov/pubmed/28986165

142. Dhonukshe-Rutten, R. A. M.,Van De Rest, O., Tieland, M. Nutrition later in life: for bone, brain and muscle function. European geriatric medicine. 2014. 5:S41https://www.cochranelibrary.com/central/doi/10.1002/central/CN-01023539/full

143. Dias, F. M.,Costa, S. O.,Pereira de Freitas, J.,Pinto Ada, C.,Vigario Pdos, S., Mainenti, M. R. Functional Capacity of Oldest Old Living in a Long-stay Institution in Rio De Janeiro, Brazil. J Phys Ther Sci. 2014. 26:1097-105 https://www.ncbi.nlm.nih.gov/pubmed/25140105

144. Dipla, K.,Makri, M.,Zafeiridis, A.,Soulas, D.,Tsalouhidou, S.,Mougios, V.,Kellis, S. An isoenergetic high-protein, moderate-fat diet does not compromise strength and fatigue during resistance exercise in women. Br J Nutr. 2008. 100:283-6 https://www.ncbi.nlm.nih.gov/pubmed/18618943 
145. Dirks, M. L.,Wall, B. T.,Kramer, I. F.,Zorenc, A. H.,Goessens, J. P.,Gijsen, A. P.,van

$\mathrm{X}$

Loon, L. J. A single session of neuromuscular electrical stimulation does not

augment postprandial muscle protein accretion. Am J Physiol Endocrinol Metab.

2016. 311:E278-85 https://www.ncbi.nlm.nih.gov/pubmed/27279248

146. Doets, E. L.,de Hoogh, I. M.,Holthuysen, N.,Wopereis, S.,Verain, M. C. D.,van den Puttelaar, J.,Hogenelst, K.,Boorsma, A.,Bouwman, E. P.,Timmer, M.,Pasman, W. J.,van Erk, M.,Reinders, M. J. Beneficial effect of personalized lifestyle advice compared to generic advice on wellbeing among Dutch seniors - An explorative study. Physiol Behav. 2019. 210:112642

https://www.ncbi.nlm.nih.gov/pubmed/31394106

147. Donaldson, M. S.,Speight, N.,Loomis, S. Fibromyalgia syndrome improved using a mostly raw vegetarian diet: an observational study. BMC Complement Altern Med. 2001. 1:7 https://www.ncbi.nlm.nih.gov/pubmed/11602026

148. Doubova, S. V.,Sanchez-Garcia, S.,Infante-Castaneda, C.,Perez-Cuevas, R. Factors associated with regular physical exercise and consumption of fruits and vegetables among Mexican older adults. BMC Public Health. 2016. 16:952 https://www.ncbi.nlm.nih.gov/pubmed/27612444

149. Draganidis, D.,Jamurtas, A. Z.,Stampoulis, T.,Laschou, V. C.,Deli, C. K., Georgakouli, K.,Papanikolaou, K.,Chatzinikolaou, A.,Michalopoulou, M., Papadopoulos, C., Tsimeas, P.,Chondrogianni, N.,Koutedakis, Y.,Karagounis, L. G., Fatouros, I. G. Disparate Habitual Physical Activity and Dietary Intake Profiles of Elderly Men with Low and Elevated Systemic Inflammation. Nutrients. 2018. 10:\#pages\# https://www.ncbi.nlm.nih.gov/pubmed/29734698

150. Drew, L. Fighting the inevitability of ageing. Nature. 2018. 555:S15-s17 https://www.ncbi.nlm.nih.gov/pubmed/29517046

151. Drks, Prevention of malnutrition and sarcopenia in aging people with proteindrinks. Http://www.who.int/trialsearch/trial2. aspx? Trialid=drks00012273. 2017. \#volume\#:\#pages\# https://www.cochranelibrary.com/central/doi/10.1002/central/CN-01889119/full

152. Duhamel, T. A.,Green, H. J.,Perco, J. G.,Ouyang, J. Comparative effects of a lowcarbohydrate diet and exercise plus a low-carbohydrate diet on muscle sarcoplasmic reticulum responses in males. Am J Physiol Cell Physiol. 2006. 291:C607-17 https://www.ncbi.nlm.nih.gov/pubmed/16707551

153. Duhamel, T. A.,Perco, J. G.,Green, H. J. Manipulation of dietary carbohydrates after prolonged effort modifies muscle sarcoplasmic reticulum responses in exercising males. Am J Physiol Regul Integr Comp Physiol. 2006. 291:R1100-10 https://www.ncbi.nlm.nih.gov/pubmed/16690765

154. Dulac, M. C.,Pion, C. H.,Lemieux, F.,Boutros El Haj,, G.,Belanger, M.,Gaudreau, P.,Chevalier, S.,Morais, J. A., Gouspillou, G.,Aubertin-Leheudre, M. Differences in muscle adaptation to a 12-week mixed power training in elderly men, depending on usual protein intake. Exp Gerontol. 2018. 104:78-85 https://www.ncbi.nlm.nih.gov/pubmed/29421607 
155. Dziuba-Słonina, A.,Rywacka, A.,Kołodziej, M. Changes in quadriceps muscle girth

$\mathrm{X} X$

$\mathrm{X}$
ater anterior cruciate ligament injury followed by electrostimulation combined with high protein diet and exercises (ARPwave method). Physiotherapy Quarterly. 2018 26:21-26

http://www.embase.com/search/results?subaction=viewrecord\&from=export\&id=L6

22973603

http://dx.doi.org/10.5114/pq.2018.74703

156. Edholm, P.,Strandberg, E.,Kadi, F. Lower limb explosive strength capacity in elderly women: effects of resistance training and healthy diet. J Appl Physiol (1985). 2017.

123:190-196 https://www.ncbi.nlm.nih.gov/pubmed/28473614

157. Farham, B. Family meals and old age. S Afr Med J. 2006. 96:606, 608 https://www.ncbi.nlm.nih.gov/pubmed/16958164

158. Farina, E. K., Thompson, L. A.,Knapik, J. J.,Pasiakos, S. M.,Lieberman, H. R., McClung, J. P. Diet Quality Is Associated with Physical Performance and Special Forces Selection. Med Sci Sports Exerc. 2019. \#volume\#:\#pages\# https://www.ncbi.nlm.nih.gov/pubmed/31436735

159. Farsijani, S.,Morais, J. A.,Payette, H.,Gaudreau, P.,Shatenstein, B.,Gray-Donald K.,Chevalier, S. Relation between mealtime distribution of protein intake and lean mass loss in free-living older adults of the NuAge study. Am J Clin Nutr. 2016. 104:694-703 https://www.ncbi.nlm.nih.gov/pubmed/27465379

160. Farsijani, S.,Payette, H.,Morais, J. A.,Shatenstein, B.,Gaudreau, P.,Chevalier, S. Even mealtime distribution of protein intake is associated with greater muscle strength, but not with 3-y physical function decline, in free-living older adults: the Quebec longitudinal study on Nutrition as a Determinant of Successful Aging (NuAge study). Am J Clin Nutr. 2017. 106:113-124

https://www.ncbi.nlm.nih gov/pubmed/28515070

161. Ferland, A.,Chateau-Degat, M. L.,Hernandez, T. L.,Eckel, R. H. Tissue-specific responses of lipoprotein lipase to dietary macronutrient composition as a predictor of weight gain over 4 years. Obesity (Silver Spring). 2012. 20:1006-11 https://www.ncbi.nlm.nih.gov/pubmed/22262159

162. Ferrand, C.,Feart, C.,Martinent, G.,Albinet, C.,Andre, N.,Audiffren, M. Dietary patterns in French home-living older adults: Results from the PRAUSE study. Arch Gerontol Geriatr. 2017. 70:180-185 https://www.ncbi.nlm.nih.gov/pubmed/28192753

163. Ferrier, K. E.,Nestel, P.,Taylor, A.,Drew, B. G.,Kingwell, B. A. Diet but not aerobic exercise training reduces skeletal muscle TNF-alpha in overweight humans. Diabetologia. 2004. 47:630-7 https://www.ncbi.nlm.nih.gov/pubmed/15298339

164. Fiatarone Singh, M. A.,Bernstein, M. A.,Ryan, A. D.,O'Neill, E. F.,Clements, K. M., Evans, W. J. The effect of oral nutritional supplements on habitual dietary quality and quantity in frail elders. J Nutr Health Aging. 2000. 4:5-12 https://www.ncbi.nlm.nih.gov/pubmed/10828934

165. Fitzgerald, J. S.,Peterson, B. J.,Wilson, P. B.,Rhodes, G. S.,Ingraham, S. J. Vitamin $\mathrm{D}$ status is associated with adiposity in male ice hockey players. Med Sci Sports Exerc. 2015. 47:655-61 https://www.ncbi.nlm.nih.gov/pubmed/24983343 
167. Foscolou, A.,Koloverou, E.,Matalas, A. L., Tyrovolas, S.,Chrysohoou, C.,Sidossis, L.,Rallidis, L.,Panagiotakos, D. B. Decomposition of Mediterranean Dietary Pattern on Successful Aging, Among Older Adults: A Combined Analysis of Two

Epidemiological Studies. J Aging Health. 2019. 31:1549-1567

https://www.ncbi.nlm.nih.gov/pubmed/29938559

168. Foscolou, A.,Magriplis, E.,Tyrovolas, S.,Soulis, G.,Bountziouka, V.,Mariolis, A. Piscopo, S.,Valacchi, G.,Anastasiou, F.,Gotsis, E.,Metallinos, G.,Tyrovola, D.

Polystipioti, A.,Polychronopoulos, E.,Matalas, A. L.,Lionis, C.,Zeimbekis, A.,Tur, J.

A.,Sidossis, L. S. Panagiotakos, D. Lifestyle determinants of healthy ageing in a

Mediterranean population: The multinational MEDIS study. Exp Gerontol. 2018.

110:35-41 https://www.ncbi.nlm.nih.gov/pubmed/29763722

169. Fougere, B.,Mazzuco, S.,Spagnolo, P.,Guyonnet, S.,Vellas, B.,Cesari, M.,Gallucci,

M. Association between the Mediterranean-style Dietary Pattern Score and

Physical Performance: Results from TRELONG Study. J Nutr Health Aging. 2016.

20:415-9 https://www.ncbi.nlm.nih.gov/pubmed/26999242

170. Franzon, K.,Byberg, L.,Sjogren, P.,Zethelius, B.,Cederholm, T.,Kilander, L.

Predictors of Independent Aging and Survival: A 16-Year Follow-Up Report in

Octogenarian Men. J Am Geriatr Soc. 2017. 65:1953-1960

https://www.ncbi.nlm.nih.gov/pubmed/28685810

171. Frassetto, L. A.,Morris, R. C., Jr.,Sellmeyer, D. E.,Sebastian, A. Adverse effects of sodium chloride on bone in the aging human population resulting from habitual

consumption of typical American diets. J Nutr. 2008. 138:419s-422s

https://www.ncbi.nlm.nih.gov/pubmed/18203914

172. Fritzen, A. M.,Lundsgaard, A. M.,Jordy, A. B.,Poulsen, S. K.,Stender, S.,Pilegaard, H.,Astrup, A.,Larsen, T. M.,Wojtaszewski, J. F.,Richter, E. A.,Kiens, B. New Nordic Diet-Induced Weight Loss Is Accompanied by Changes in Metabolism and AMPK Signaling in Adipose Tissue. J Clin Endocrinol Metab. 2015. 100:3509-19

https://www.ncbi.nlm.nih.gov/pubmed/26126206

173. Fukuda, Y.,Yamamoto, K.,Hirao, M.,Nishikawa, K.,Nagatsuma, Y.,Nakayama, T., Tanikawa, S.,Maeda, S.,Uemura, M.,Miyake, M.,Hama, N.,Miyamoto, A.,Ikeda, M., Nakamori, S.,Sekimoto, M.,Fujitani, K.,Tsujinaka, T. Sarcopenia is associated with severe postoperative complications in elderly gastric cancer patients undergoing gastrectomy. Gastric Cancer. 2016. 19:986-93 https://www.ncbi.nlm.nih.gov/pubmed/26407875

174. Galbreath, M.,Campbell, B.,LaBounty, P.,Bunn, J.,Dove, J.,Harvey, T.,Hudson, G., Gutierrez, J. L.,Levers, K., Galvan, E.,Jagim, A., Greenwood, L.,Cooke, M. B., Greenwood, M.,Rasmussen, C.,Kreider, R. B. Effects of Adherence to a Higher Protein Diet on Weight Loss, Markers of Health, and Functional Capacity in Olde Women Participating in a Resistance-Based Exercise Program. Nutrients. 2018. 10:\#pages\# https://www.ncbi.nlm.nih.gov/pubmed/30103509

175. Gale, C. R.,Martyn, C. N.,Kellingray, S.,Eastell, R.,Cooper, C. Intrauterine programming of adult body composition. J Clin Endocrinol Metab. 2001. 86:267-72 https://www.ncbi.nlm.nih.gov/pubmed/11232011 
176. Galesi, L. F.,Leandro-Merhi, V. A.,de Oliveira, M. R. Association between indicators

$\mathrm{X}$

of dementia and nutritional status in institutionalised older people. Int J Older People Nurs. 2013. 8:236-43 https://www.ncbi.nlm.nih.gov/pubmed/22394646

177. Gallucci, M.,Mazzuco, S.,Ongaro, F.,Di Giorgi, E.,Mecocci, P.,Cesari, M.,Albani, D., Forloni, G. L.,Durante, E.,Gajo, G. B.,Zanardo, A.,Siculi, M.,Caberlotto, L.,Regini, C. Body mass index, lifestyles, physical performance and cognitive decline: the "Treviso Longeva (TRELONG)" study. J Nutr Health Aging. 2013. 17:378-84 https://www.ncbi.nlm.nih.gov/pubmed/23538662

178. Gallucci, M.,Pallucca, C.,Di Battista, M. E.,Fougereand, B.,Grossi, E. Artificial Neural Networks Help to Better Understand the Interplay Between Cognition, Mediterranean Diet, and Physical Performance: Clues from TRELONG Study. J Alzheimers Dis. 2019. \#volume\#:\#pages\#

https://www.ncbi.nlm.nih.gov/pubmed/31524170

179. Garduno-Diaz, S. D.,Khokhar, S. South Asian dietary patterns and their association with risk factors for the metabolic syndrome. J Hum Nutr Diet. 2013. 26:145-55 https://www.ncbi.nlm.nih.gov/pubmed/22943473

180. Garthe, I.,Raastad, T.,Refsnes, P. E.,Sundgot-Borgen, J. Effect of nutritional intervention on body composition and performance in elite athletes. Eur J Sport Sci. 2013. 13:295-303 https://www.ncbi.nlm.nih.gov/pubmed/23679146

181. Gazzani, D.,Zamboni, F.,Spelta, F.,Ferrari, P.,Mattioli, V.,Cazzoletti, L.,Zanolin, E., Tardivo, S.,Ferrari, M. Vegetable but not animal protein intake is associated to a better physical performance: a study on a general population sample of adults. Food Nutr Res. 2019. 63:\#pages\# https://www.ncbi.nlm.nih.gov/pubmed/31565042

182. Geerling, B. J.,Badart-Smook, A.,Stockbrugger, R. W.,Brummer, R. J. Comprehensive nutritional status in recently diagnosed patients with inflammatory bowel disease compared with population controls. Eur J Clin Nutr. 2000. 54:514-21 https://www.ncbi.nlm.nih.gov/pubmed/10878655

183. Geirsdottir, O. G.,Arnarson, A.,Ramel, A.,Jonsson, P. V.,Thorsdottir, I. Dietary protein intake is associated with lean body mass in community-dwelling older adults. Nutr Res. 2013. 33:608-12 https://www.ncbi.nlm.nih.gov/pubmed/23890349

184. Genaro Pde, S.,Pinheiro Mde, M.,Szejnfeld, V. L.,Martini, L. A. Dietary protein intake in elderly women: association with muscle and bone mass. Nutr Clin Pract. 2015. 30:283-9 https://www.ncbi.nlm.nih.gov/pubmed/25107954

185. Germain, L.,Latarche, C.,Kesse-Guyot, E.,Galan, P.,Hercberg, S.,Briancon, S. Does compliance with nutrition guidelines lead to healthy aging? A quality-of-life approach. J Acad Nutr Diet. 2013. 113:228-40.e1-2 https://www.ncbi.nlm.nih.gov/pubmed/23351626

186. Ghloum, K.,Hajji, S. Comparison of diet consumption, body composition and lipoprotein lipid values of Kuwaiti fencing players with international norms. J Int Soc Sports Nutr. 2011. 8:13 https://www.ncbi.nlm.nih.gov/pubmed/21992447

187. Gillen, J. B., Trommelen, J.,Wardenaar, F. C.,Brinkmans, N. Y.,Versteegen, J. J., Jonvik, K. L.,Kapp, C., de Vries, J.,van den Borne, J. J.,Gibala, M. J.,van Loon, L. J. Dietary Protein Intake and Distribution Patterns of Well-Trained Dutch Athletes. Int J Sport Nutr Exerc Metab. 2017. 27:105-114

https://www.ncbi.nlm.nih.gov/pubmed/27710150 
Gingrich, A., Rennekamp, R.,Brandl, B.,Skurk, T.,Hauner, H.,Sieber, C. C., Volkert,
D.,Kiesswetter, E. Do Aspects of Protein Intake Vary Across the Week in Healthy Community-Dwelling Older Adults?-An enable Study. Nutrients. 2018. 10:\#pages\# https://www.ncbi.nlm.nih.gov/pubmed/30177590

189. Gjevestad, G. O., Ottestad, I.,Biong, A. S.,Iversen, P. O., Retterstol, K.,Raastad, T., Skalhegg, B. S.,Ulven, S. M.,Holven, K. B. Consumption of protein-enriched milk has minor effects on inflammation in older adults-A 12-week double-blind

randomized controlled trial. Mech Ageing Dev. 2017. 162:1-8

https://www.ncbi.nlm.nih.gov/pubmed/28163108

190. Glabska, D.,Cackowska, K.,Guzek, D. Comparison of the Body Composition of Caucasian Young Normal Body Mass Women, Measured in the Follicular Phase, Depending on the Carbohydrate Diet Level. Medicina (Kaunas). 2018. 54:\#pages\# https://www.ncbi.nlm.nih.gov/pubmed/30563184

191. Goff, L. M.,Bell, J. D.,So, P. W.,Dornhorst, A.,Frost, G. S. Veganism and its relationship with insulin resistance and intramyocellular lipid. Eur J Clin Nutr. 2005. 59:291-8 https://www.ncbi.nlm.nih.gov/pubmed/15523486

192. Gojda, J.,Patkova, J.,Jacek, M.,Potockova, J.,Trnka, J.,Kraml, P.,Andel, M. Higher insulin sensitivity in vegans is not associated with higher mitochondrial density. Eur J Clin Nutr. 2013. 67:1310-5 https://www.ncbi.nlm.nih.gov/pubmed/24149445

193. Gomez-Arbelaez, D.,Bellido, D.,Castro, A. I.,Ordonez-Mayan, L.,Carreira, J., Galban, C.,Martinez-Olmos, M. A., Crujeiras, A. B.,Sajoux, I., Casanueva, F. F. Body Composition Changes After Very-Low-Calorie Ketogenic Diet in Obesity Evaluated by 3 Standardized Methods. J Clin Endocrinol Metab. 2017. 102:488-498 https://www.ncbi.nlm.nih.gov/pubmed/27754807

194. Gonzalez-Reimers, E.,Alvisa-Negrin, J.,Santolaria-Fernandez, F.,Candelaria Martin-Gonzalez, M.,Hernandez-Betancor, I.,Fernandez-Rodriguez, C. M.,VinaRodriguez, J.,Gonzalez-Diaz, A. Vitamin D and nutritional status are related to bone fractures in alcoholics. Alcohol Alcohol. 2011. 46:148-55 https://www.ncbi.nlm.nih.gov/pubmed/21248027

195. Goodpaster, B. H.,Katsiaras, A.,Kelley, D. E. Enhanced fat oxidation through physical activity is associated with improvements in insulin sensitivity in obesity Diabetes. 2003. 52:2191-7 https://www.ncbi.nlm.nih.gov/pubmed/12941756

196. Gopinath, B.,Flood, V. M.,Burlutksy, G.,Liew, G.,Mitchell, P. Carbohydrate nutrition variables and risk of disability in instrumental activities of daily living. Eur $J$ Nutr. 2018. \#volume\#:\#pages\# https://www.ncbi.nlm.nih.gov/pubmed/30448879

197. Gopinath, B.,Russell, J.,Flood, V. M.,Burlutsky, G.,Mitchell, P. Adherence to dietary guidelines positively affects quality of life and functional status of older adults. $J$ Acad Nutr Diet. 2014. 114:220-9 https://www.ncbi.nlm.nih.gov/pubmed/24239401

198. Gordon, M. M.,Bopp, M. J.,Easter, L.,Miller, G. D.,Lyles, M. F.,Houston, D. K., Nicklas, B. J.,Kritchevsky, S. B. Effects of dietary protein on the composition of weight loss in post-menopausal women. J Nutr Health Aging. 2008. 12:505-9 https://www.ncbi.nlm.nih.gov/pubmed/18810296

199. Gorgey, A. S.,Dolbow, D. R.,Cifu, D. X.,Gater, D. R. Neuromuscular electrical stimulation attenuates thigh skeletal muscles atrophy but Xt trunk muscles after spinal cord injury. J Electromyogr Kinesiol. 2013. 23:977-84

https://www.ncbi.nlm.nih.gov/pubmed/23683374

\section{$X$}

$\mathrm{X}$

$X$

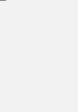

\section{$x$}


201. Grabovac, I.,Haider, S.,Winzer, E.,Kapan, A.,Schindler, K. E.,Lackinger, C.,Dorner T. E. Changes in health parameters in older lay volunteers who delivered a lifestyle-based program to frail older people at home. Wien Klin Wochenschr. 2018. 130:637-644 https://www.ncbi.nlm.nih.gov/pubmed/30094663

202. Graciani, A., Garcia-Esquinas, E.,Lopez-Garcia, E.,Banegas, J. R.,RodriguezArtalejo, F. Ideal Cardiovascular Health and Risk of Frailty in Older Adults. Circ Cardiovasc Qual Outcomes. 2016. 9:239-45

https://www.ncbi.nlm.nih.gov/pubmed/27166207

203. Granic, A.,Jagger, C.,Davies, K.,Adamson, A.,Kirkwood, T.,Hill, T. R.,Siervo, M., Mathers, J. C.,Sayer, A. A. Effect of Dietary Patterns on Muscle Strength and Physical Performance in the Very Old: Findings from the Newcastle 85+ Study. PLoS One. 2016. 11:e0149699 https://www.ncbi.nlm.nih.gov/pubmed/26934360

204. Granic, A.,Mendonça, N.,Sayer, A. A.,Hill, T. R.,Davies, K.,Adamson, A.,Siervo, M. Mathers, J. C.,Jagger, C. Low protein intake, muscle strength and physical performance in the very old: The Newcastle 85+ Study. Clinical Nutrition. 2018. $37: 2260-2270$

http://www.embase.com/search/results?subaction=viewrecord\&from=export\&id=L6 19465343

http://dx.doi.org/10.1016/j.clnu.2017.11.005

205. Gray-Donald, K.,St-Arnaud-McKenzie, D.,Gaudreau, P.,Morais, J. A.,Shatenstein B.,Payette, H. Protein intake protects against weight loss in healthy communitydwelling older adults. J Nutr. 2014. 144:321-6

https://www.ncbi.nlm.nih.gov/pubmed/24357473

206. Green, M. W.,Elliman, N. A.,Kretsch, M. J. Weight loss strategies, stress, and cognitive function: supervised versus unsupervised dieting.

Psychoneuroendocrinology. 2005. 30:908-18

https://www.ncbi.nlm.nih.gov/pubmed/15970392

207. Guligowska, A.,Piglowska, M.,Fife, E.,Kostka, J.,Soltysik, B. K.,Kroc, L.,Kostka, T. Inappropriate nutrients intake is associated with lower functional status and inferior quality of life in older adults with depression. Clin Interv Aging. 2016. 11:1505-1517 https://www.ncbi.nlm.nih.gov/pubmed/27822023

208. Hagan, K. A.,Chiuve, S. E.,Stampfer, M. J.,Katz, J. N.,Grodstein, F. Greater Adherence to the Alternative Healthy Eating Index Is Associated with Lower Incidence of Physical Function Impairment in the Nurses' Health Study. J Nutr. 2016. 146:1341-7 https://www.ncbi.nlm.nih.gov/pubmed/27170727

209. Hagan, K. A.,Grodstein, F. The Alternative Healthy Eating Index and Physical Function Impairment in Men. $J$ Nutr Health Aging. 2019. 23:459-465 https://www.ncbi.nlm.nih.gov/pubmed/31021363

210. Hai, S.,Wang, H.,Cao, L.,Liu, P.,Zhou, J.,Yang, Y.,Dong, B. Association between sarcopenia with lifestyle and family function among community-dwelling Chinese aged 60 years and older. BMC Geriatr. 2017. 17:187 https://www.ncbi.nlm.nih.gov/pubmed/28821239 
Resistance training is associated with spontaneous changes in aerobic physical activity but not overall diet quality in adults with prediabetes. Physiol Behav. 2017. 177:49-56 https://www.ncbi.nlm.nih.gov/pubmed/28414072

212. Han, P.,Zhao, J.,Guo, Q.,Wang, J.,Zhang, W.,Shen, S.,Wang, X.,Dong, R.,Ma, Y., Kang, L.,Fu, L.,Jia, L.,Han, X.,He, Z.,Bao, Y.,Wang, L.,Niu, K. Incidence, risk factors, and the protective effect of high body mass index against sarcopenia in suburb-dwelling elderly Chinese populations. Journal of Nutrition, Health and Aging. 2016. 20:1056-1060

http://www.embase.com/search/results?subaction=viewrecord\&from=export\&id=L6 08644619

http://dx.doi.org/10.1007/s12603-016-0704-3

213. Haub, M. D.,Wells, A. M.,Campbell, W. W. Beef and soy-based food supplements differentially affect serum lipoprotein-lipid profiles because of changes in carbohydrate intake and novel nutrient intake ratios in older men who resistivetrain. Metabolism. 2005. 54:769-74

https://www.ncbi.nlm.nih.gov/pubmed/15931612

214. Haub, M. D.,Wells, A. M.,Tarnopolsky, M. A.,Campbell, W. W. Effect of protein source on resistive-training-induced changes in body composition and muscle size in older men. Am J Clin Nutr. 2002. 76:511-7

https://www.ncbi.nlm.nih.gov/pubmed/12197993

215. Hayashi, F.,Matsumoto, Y.,Momoki, C.,Yuikawa, M.,Okada, G.,Hamakawa, E., Kawamura, E.,Hagihara, A., Toyama, M.,Fujii, H.,Kobayashi, S.,Iwai, S.,Morikawa, H.,Enomoto, M.,Tamori, A.,Kawada, N.,Habu, D. Physical inactivity and insufficient dietary intake are associated with the frequency of sarcopenia in patients with compensated viral liver cirrhosis. Hepatol Res. 2013. 43:1264-75 https://www.ncbi.nlm.nih.gov/pubmed/23489325

216. Hayashi, F.,Momoki, C.,Yuikawa, M.,Simotani, Y.,Kawamura, E.,Hagihara, A.,Fujii, H.,Kobayashi, S.,Iwai, S.,Morikawa, H., EXmoto, M.,Tamori, A., Kawada, N.,Ohfuji, S.,Fukusima, W.,Habu, D. Nutritional status in relation to lifestyle in patients with compensated viral cirrhosis. World J Gastroenterol. 2012. 18:5759-70 https://www.ncbi.nlm.nih.gov/pubmed/23155318

217. Hazreen, M. A.,Su, T. T.,Jalaludin, M. Y.,Dahlui, M.,Chinna, K.,Ismail, M.,Murray, L.,Cantwell, M.,Al Sadat, N. An exploratory study on risk factors for chronic noncommunicable diseases among adolescents in Malaysia: overview of the Malaysian Health and Adolescents Longitudinal Research Team study (The MyHeART study). BMC Public Health. 2014. 14 Suppl 3:S6 https://www.ncbi.nlm.nih.gov/pubmed/25437068

218. Hegerova, P.,Dedkova, Z.,Sobotka, L. Early nutritional support and physiotherapy improved long-term self-sufficiency in acutely ill older patients. Nutrition. 2015. 31:166-70 https://www.ncbi.nlm.nih.gov/pubmed/25466662

219. Helms, E. R.,Zinn, C.,Rowlands, D. S.,Naidoo, R.,Cronin, J. High-protein, low-fat, short-term diet results in less stress and fatigue than moderate-protein moderate-fat diet during weight loss in male weightlifters: a pilot study. Int J Sport Nutr Exerc Metab. 2015. 25:163-70 https://www.ncbi.nlm.nih.gov/pubmed/25028958

220. Hermanussen, H. H.,Menendez, M. E.,Chen, N. C.,Ring, D.,Vranceanu, A. M. Predictors of Upper-Extremity Physical Function in Older Adults. Arch Bone Jt Surg. 2016. 4:359-365 https://www.ncbi.nlm.nih.gov/pubmed/27847850 
221. Herrema, A. L.,Westerman, M. J.,van Dongen, E. J. I.,Kudla, U.,Veltkamp, M. Combined Protein-Rich Diet With Resistance Exercise Intervention to Counteract Sarcopenia: A Qualitative Study on Drivers and Barriers of Compliance. J Aging Phys Act. 2018. 26:106-113 https://www.ncbi.nlm.nih.gov/pubmed/28595022

222. Hietavala, E. M.,Puurtinen, R.,Kainulainen, H.,Mero, A. A. Low-protein vegetarian diet does not have a short-term effect on blood acid-base status but raises oxygen consumption during submaximal cycling. J Int Soc Sports Nutr. 2012. 9:50 https://www.ncbi.nlm.nih.gov/pubmed/23181739

223. Hirsch, K. R.,Tweedell, A. J.,Kleinberg, C. R., Gerstner, G. R.,Barnette, T. J.,Mota, J. A.,Smith-Ryan, A. E.,Ryan, E. D. The Influence of Habitual Protein Intake on Body Composition and Muscular Strength in Career Firefighters. J Am Coll Nutr. 2018. 37:620-626 https://www.ncbi.nlm.nih.gov/pubmed/29702029

224. Ho, R. C.,Davy, K. P.,Hickey, M. S.,Summers, S. A.,Melby, C. L. Behavioral, metabolic, and molecular correlates of lower insulin sensitivity in MexicanAmericans. Am J Physiol Endocrinol Metab. 2002. 283:E799-808 https://www.ncbi.nlm.nih.gov/pubmed/12217898

225. Hodge, A. M.,O'Dea, K.,English, D. R.,Giles, G. G.,Flicker, L. Dietary patterns as predictors of successful ageing. J Nutr Health Aging. 2014. 18:221-7 https://www.ncbi.nlm.nih.gov/pubmed/24626747

226. Holm, L.,Nordsborg, N. B. Supplementing a normal diet with protein yields a moderate improvement in the robust gains in muscle mass and strength induced by resistance training in older individuals. Am J Clin Nutr. 2017. 106:971-972 https://www.ncbi.nlm.nih.gov/pubmed/28877892

227. Holwerda, A. M.,Kouw, I. W.,Trommelen, J.,Halson, S. L.,Wodzig, W. K.,Verdijk, L. B.,van Loon, L. J. Physical Activity Performed in the Evening Increases the Overnight Muscle Protein Synthetic Response to Presleep Protein Ingestion in Older Men. J Nutr. 2016. 146:1307-14 https://www.ncbi.nlm.nih.gov/pubmed/27281811

228. Hopkins, M.,Finlayson, G.,Duarte, C.,Whybrow, S.,Ritz, P.,Horgan, G. W.,Blundell, J. E.,Stubbs, R. J. Modelling the associations between fat-free mass, resting metabolic rate and energy intake in the context of total energy balance. Int $J$ Obes (Lond). 2016. 40:312-8 https://www.ncbi.nlm.nih.gov/pubmed/26278004

229. Houston, D. K.,Nicklas, B. J.,Ding, J.,Harris, T. B., Tylavsky, F. A.,Newman, A. B. Lee, J. S.,Sahyoun, N. R., Visser, M.,Kritchevsky, S. B. Dietary protein intake is associated with lean mass change in older, community-dwelling adults: the Health Aging, and Body Composition (Health ABC) Study. Am J Clin Nutr. 2008. 87:150-5 https://www.ncbi.nlm.nih.gov/pubmed/18175749

230. Houston, D. K.,Stevens, J.,Cai, J.,Haines, P. S. Dairy, fruit, and vegetable intakes and functional limitations and disability in a biracial cohort: the Atherosclerosis Risk in Communities Study. Am J Clin Nutr. 2005. 81:515-22 https://www.ncbi.nlm.nih.gov/pubmed/15699243

231. Houston, D. K.,Tooze, J. A.,Garcia, K.,Visser, M.,Rubin, S.,Harris, T. B.,Newman, A. B.,Kritchevsky, S. B. Protein Intake and Mobility Limitation in CommunityDwelling Older Adults: the Health ABC Study. J Am Geriatr Soc. 2017. 65:1705-

$\mathrm{X}$

1711 https://www.ncbi.nlm.nih.gov/pubmed/28306154

$\mathrm{x} \quad \mathrm{x}$




$\mathrm{X}$

x
Integrity in Aging: The Framingham Heart Study Offspring. J Gerontol A Biol Sc Med Sci. 2018. \#volume\#:\#pages\# https://www.ncbi.nlm.nih.gov/pubmed/30247514

233. Hu, S.,Gu, Y.,Lu, Z.,Zhang, Q.,Liu, L.,Meng, G., Yao, Z.,Wu, H.,Bao, X., Chi, V. T. Q.,Zhang, S.,Liu, M.,Wang, Y.,Wang, L.,Zheng, L.,Wang, X.,Tian, C.,Fu, J.,Sun, S., Zhou, M.,Jia, Q.,Song, K.,Sun, Z.,Niu, K. Relationship Between Grip Strength and Prediabetes in a Large-Scale Adult Population. Am J Prev Med. 2019. 56:844-851 https://www.ncbi.nlm.nih.gov/pubmed/31003803

234. Hu, T. Y.,Chen, Y. C.,Lin, P.,Shih, C. K.,Bai, C. H.,Yuan, K. C.,Lee, S. Y.,Chang, J. S. Testosterone-Associated Dietary Pattern Predicts Low Testosterone Levels and Hypogonadism. Nutrients. 2018. 10:\#pages\# https://www.ncbi.nlm.nih.gov/pubmed/30453566

235. Huang, Y. C.,Wahlqvist, M. L.,Lo, Y. T. C.,Lin, C., Chang, H. Y.,Lee, M. S. A noninvasive modifiable Healthy Ageing Nutrition Index (HANI) predicts longevity in freeliving older Taiwanese. Scientific reports. 2018. 8:7113

http://www.embase.com/search/results?subaction=viewrecord\&from=export\&id=L6 29354978

http://dx.doi.org/10.1038/s41598-018-24625-3

236. Hughes, S. L.,Tussing-Humphreys, L.,Schiffer, L.,Smith-Ray, R.,Marquez, D. X., DeMott, A. D.,Berbaum, M. L.,Fitzgibbon, M. L. Fit \& Strong! Plus Trial Outcomes for Obese Older Adults with Osteoarthritis. Gerontologist. 2018. \#volume\#:\#pages\# https://www.ncbi.nlm.nih.gov/pubmed/30476065

237. Hutasuhut, F.,Ryoto, V. Associations between muscle grip strength with age, body mass index, waist-to-hip ratio, level of independent, physical activity level and macronutrient intake in elderly women. Pakistan Journal of Nutrition. 2014. 13:409414

http://www.embase.com/search/results?subaction=viewrecord\&from=export\&id=L6 00386359

http://dx.doi.org/10.3923/pjn.2014.409.414

238. Hutchins-Wiese, H. L.,Kleppinger, A.,Annis, K.,Liva, E.,Lammi-Keefe, C. J., Durham, H. A.,Kenny, A. M. The impact of supplemental n-3 long chain polyunsaturated fatty acids and dietary antioxidants on physical performance in postmenopausal women. J Nutr Health Aging. 2013. 17:76-80 https://www.ncbi.nlm.nih.gov/pubmed/23299384

239. Huuskonen, J.,Vaisanen, S. B.,Kroger, H.,Jurvelin, C.,Bouchard, C.,Alhava, E., Rauramaa, R. Determinants of bone mineral density in middle aged men: a population-based study. Osteoporos Int. 2000. 11:702-8 https://www.ncbi.nlm.nih.gov/pubmed/11095174

240. Iglay, H. B.,Apolzan, J. W.,Gerrard, D. E.,Eash, J. K.,Anderson, J. C.,Campbell, W. $W$. Moderately increased protein intake predominately from egg sources does not influence whole body, regional, or muscle composition responses to resistance training in older people. J Nutr Health Aging. 2009. 13:108-14 https://www.ncbi.nlm.nih.gov/pubmed/19214338

241. Irazusta, A., Gil, S.,Ruiz, F.,Gondra, J., Jauregi, A.,Irazusta, J.,Gil, J. Exercise, physical fitness, and dietary habits of first-year female nursing students. Biol Res Nurs. 2006. 7:175-86 https://www.ncbi.nlm.nih.gov/pubmed/16552945 
A. T. Dietary protein intake is associated with better physical function and muscle strength among elderly women. Br J Nutr. 2016. 115:1281-91

https://www.ncbi.nlm.nih.gov/pubmed/26857389

243. Isanejad, M.,Mursu, J.,Sirola, J.,Kroger, H.,Rikkonen, T.,Tuppurainen, M.,Erkkila A. T. Association of protein intake with the change of lean mass among elderly women: The Osteoporosis Risk Factor and Prevention - Fracture Prevention Study (OSTPRE-FPS). J Nutr Sci. 2015. 4:e41

https://www.ncbi.nlm.nih.gov/pubmed/26793306

244. Izumotani, K.,Hagiwara, S., Izumotani, T.,Miki, T.,Morii, H.,Nishizawa, Y. Risk factors for osteoporosis in men. J Bone Miner Metab. 2003. 21:86-90 https://www.ncbi.nlm.nih.gov/pubmed/12601572

245. Jacob, M. E.,Yee, L. M.,Diehr, P. H.,Arnold, A. M.,Thielke, S. M.,Chaves, P. H Gobbo, L. D.,Hirsch, C.,Siscovick, D.,Newman, A. B. Can a Healthy Lifestyle Compress the Disabled Period in Older Adults?. J Am Geriatr Soc. 2016. 64:1952$1961 \mathrm{https}: / /$ www.ncbi.nlm.nih.gov/pubmed/27603679

246. Jadczak, A. D.,Luscombe-Marsh, N.,Taylor, P.,Barnard, R.,Makwana, N., Visvanathan, R. The EXPRESS Study: exercise and Protein Effectiveness Supplementation Study supporting autonomy in community dwelling frail older people study protocol for a randomized controlled pilot and feasibility study. Pilot and feasibility studies. 2018. 4:\#pages\#

https://www.cochranelibrary.com/central/doi/10.1002/central/CN-01755477/full

247. Jarvis, M.,McNaughton, L.,Seddon, A.,Thompson, D. The acute 1-week effects of the Zone diet on body composition, blood lipid levels, and performance in recreational endurance athletes. J Strength Cond Res. 2002. 16:50-7 https://www.ncbi.nlm.nih.gov/pubmed/11834107

248. Jenkinson, C. M.,Doherty, M.,Avery, A. J.,Read, A.,Taylor, M. A.,Sach, T. H. Silcocks, P.,Muir, K. R. Effects of dietary intervention and quadriceps strengthening exercises on pain and function in overweight people with knee pain: randomised controlled trial. BMJ (clinical research ed.). 2009. 339:b3170

https://www.cochranelibrary.com/central/doi/10.1002/central/CN-00719642/full

249. Jensen, G. L.,Roy, M. A.,Buchanan, A. E.,Berg, M. B. Weight loss intervention for obese older women: improvements in performance and function. Obes Res. 2004. 12:1814-20 https://www.ncbi.nlm.nih.gov/pubmed/15601977

250. Jeong, G. W.,Kim, Y. J.,Park, S.,Kim, H.,Kwon, O. Associations of recommended food score and physical performance in Korean elderly. BMC Public Health. 2019. 19:128 https://www.ncbi.nlm.nih.gov/pubmed/30700281

251. Jin, Y..Tanaka, T.,Ma, Y.,Bandinelli, S.,Ferrucci, L.,Talegawkar, S. A. Cardiovascular Health Is Associated With Physical Function Among Older Community Dwelling Men and Women. J Gerontol A Biol Sci Med Sci. 2017. 72:1710-1716 https://www.ncbi.nlm.nih.gov/pubmed/28184412

252. Jo, E.,Worts, P. R.,Elam, M. L.,Brown, A. F.,Khamoui, A. V.,Kim, D. H.,Yeh, M. C. Ormsbee, M. J.,Prado, C. M.,Cain, A.,Snyder, K.,Kim, J. S. Resistance training during a 12-week protein supplemented VLCD treatment enhances weight-loss outcomes in obese patients. Clin Nutr. 2019. 38:372-382 https://www.ncbi.nlm.nih.gov/pubmed/29352654 
253. Johnson, C. S.,Mahon, A.,McLeod, W. Nutritional, functional and psychosocial $\mathrm{X}$ correlates of disability among older adults. J Nutr Health Aging. 2006. 10:45-50 https://www.ncbi.nlm.nih.gov/pubmed/16453057

254. Johnson, L. J.,McCool, A. C. Dietary intake and nutritional status of older adult homeless women: a pilot study. J Nutr Elder. 2003. 23:1-21 https://www.ncbi.nlm.nih.gov/pubmed/14650550

255. Josse, A. R.,Atkinson, S. A.,Tarnopolsky, M. A.,Phillips, S. M. Increased consumption of dairy foods and protein during diet- and exercise-induced weight loss promotes fat mass loss and lean mass gain in overweight and obese premenopausal women. J Nutr. 2011. 141:1626-34 https://www.ncbi.nlm.nih.gov/pubmed/21775530

256. Josse, A. R.,Atkinson, S. A.,Tarnopolsky, M. A.,Phillips, S. M. Consumption of higher dairy and dietary protein during diet- and exercise-induced weight loss promotes a metabolically favourable body composition change in overweight and obese young women. FASEB journal. 2011. 25:\#pages\# https://www.cochranelibrary.com/central/doi/10.1002/central/CN-01033363/full

257. Joy, J. M.,Lowery, R. P.,Wilson, J. M.,Purpura, M.,De Souza, E. O.,Wilson, S. M. Kalman, D. S.,Dudeck, J. E.,Jager, R. The effects of 8 weeks of whey or rice protein supplementation on body composition and exercise performance. Nutr $\mathrm{J}$. 2013. 12:86 https://www.ncbi.nlm.nih.gov/pubmed/23782948

258. Jprn, Umin A cross-over trial for preventing frailty in the community dwelling olde adults. Http://www. who.int/trialsearch/trial2.aspx? Trialid=jprn-umin000011435 2013. \#volume\#:\#pages\# https://www.cochranelibrary.com/central/doi/10.1002/central/CN-01796456/full

259. Jprn, Umin A community-based intervention using TAKE10! program to improve dietary habit and to promote physical activity among the elderly: a cluster randomizes trail. Http://www.who.int/trialsearch/trial2.aspx? Trialid=jprnumin000007357. 2012. \#volume\#:\#pages\# https://www.cochranelibrary.com/central/doi/10.1002/central/CN-01830944/full

260. Jung, M.,Park, S.,Kim, H.,Kwon, O. Association of Diet Quality with Low Muscle Mass-Function in Korean Elderly. Int J Environ Res Public Health. 2019. 16:\#pages\# https://www.ncbi.nlm.nih.gov/pubmed/31370245

261. Jyväkorpi, S. K.,Urtamo, A.,Pitkälä, K. H.,Strandberg, T. E. Nutrition, daily walking and resilience are associated with physical function in the oldest old men. Journal of Nutrition, Health and Aging. 2018. 22:1176-1182 http://www.embase.com/search/results?subaction=viewrecord\&from=export\&id=L6 26914180 http://dx.doi.org/10.1007/s12603-018-1136-z

262. Kang, H. J.,Jun, D. W.,Lee, S. M.,Jang, E. C.,Cho, Y. K. Low salt and low calorie diet does not reduce more body fat than same calorie diet: a randomized controlled study. Oncotarget. 2018. 9:8521-8530

https://www.ncbi.nlm.nih.gov/pubmed/29492213

263. Karelis, A. D.,Lavoie, M. E.,Fontaine, J.,Messier, V.,Strychar, I.,Rabasa-Lhoret, R., $\quad$ X Doucet, E. Anthropometric, metabolic, dietary and psychosocial profiles of underreporters of energy intake: a doubly labeled water study among overweight/obese postmenopausal women--a Montreal Ottawa New Emerging Team study. Eur J Clin Nutr. 2010. 64:68-74

https://www.ncbi.nlm.nih.gov/pubmed/19756035 

cysteine-rich whey protein (immunocal(R)) supplementation in combination with

$\mathrm{X}$

resistance training on muscle strength and lean body mass in non-frail elderly

subjects: a randomized, double-blind controlled study. J Nutr Health Aging. 2015.

19:531-6 https://www.ncbi.nlm.nih.gov/pubmed/25923482

265. Keller, H. H. Reliance on others for food-related activities of daily living. $J$ Nutr Elder. 2005. 25:43-59 https://www.ncbi.nlm.nih.gov/pubmed/16891262

266. Kemmler, W.,Teschler, M.,Weissenfels, A.,Bebenek, M.,von Stengel, S.,Kohl, M., Freiberger, E.,Goisser, S.,Jakob, F.,Sieber, C.,Engelke, K. Whole-body electromyostimulation to fight sarcopenic obesity in community-dwelling older women at risk. Resultsof the randomized controlled FORMOsA-sarcopenic obesity study. Osteoporosis International. 2016. 27:3261-3270

http://www.embase.com/search/results?subaction=viewrecord\&from=export\&id=L6 12665826

http://dx.doi.org/10.1007/s00198-016-3662-z

267. Kephart, W. C.,Pledge, C. D.,Roberson, P. A.,Mumford, P. W.,Romero, M. A., Mobley, C. B.,Martin, J. S.,Young, K. C.,Lowery, R. P.,Wilson, J. M.,Huggins, K.

W.,Roberts, M. D. The Three-Month Effects of a Ketogenic Diet on Body

Composition, Blood Parameters, and Performance Metrics in CrossFit Trainees: A

Pilot Study. Sports (Basel). 2018. 6:\#pages\#

https://www.ncbi.nlm.nih.gov/pubmed/29910305

268. Kido, Y. The Issue of Nutrition in an Aging Society. J Nutr Sci Vitaminol (Tokyo). 2015. 61 Suppl:S176-7 https://www.ncbi.nlm.nih.gov/pubmed/26598846

269. Kim, I. Y.,Schutzler, S.,Schrader, A. M.,Spencer, H. J.,Azhar, G.,Wolfe, R. R., Ferrando, A. A. Protein intake distribution pattern does not affect anabolic response, lean body mass, muscle strength or function over 8 weeks in older adults: A randomized-controlled trial. Clin Nutr. 2018. 37:488-493

https://www.ncbi.nlm.nih.gov/pubmed/28318687

270. Kim, I. Y.,Schutzler, S.,Schrader, A.,Spencer, H.,Kortebein, P.,Deutz, N. E.,Wolfe, R. R.,Ferrando, A. A. Quantity of dietary protein intake, but not pattern of intake, affects net protein balance primarily through differences in protein synthesis in older adults. Am J Physiol Endocrinol Metab. 2015. 308:E21-8 https://www.ncbi.nlm.nih.gov/pubmed/25352437

271. Kim, J.,Jung, M.,Hong, Y. P.,Park, J. D.,Choi, B. S. Physical activity in adolescence has a positive effect on bone mineral density in young men. $J$ Prev Med Public Health. 2013. 46:89-95 https://www.ncbi.nlm.nih.gov/pubmed/23573373

272. Kimokoti, R. W.,Newby, P. K.,Gona, P.,Zhu, L.,Jasuja, G. K.,Pencina, M. J., McKeon-O'Malley, C.,Fox, C. S.,D'Agostino, R. B.,Millen, B. E. Diet quality, physical activity, smoking status, and weight fluctuation are associated with weight change in women and men. $J$ Nutr. 2010.140:1287-93 https://www.ncbi.nlm.nih.gov/pubmed/20484553

273. Kirk, B.,Mooney, K.,Amirabdollahian, F.,Khaiyat, O. Exercise and Dietary-Protein as a Countermeasure to Skeletal Muscle Weakness: Liverpool Hope University Sarcopenia Aging Trial (LHU-SAT). Front Physiol. 2019. 10:445 https://www.ncbi.nlm.nih.gov/pubmed/31133863

$\mathrm{X}$

.


274. Kirwan, R. D.,Kordick, L. K.,McFarland, S.,Lancaster, D.,Clark, K.,Miles, M. P Dietary, anthropometric, blood-lipid, and performance patterns of American College Football Players during 8 weeks of training. Int J Sport Nutr Exerc Metab. 2012. 22:444-51 https://www.ncbi.nlm.nih.gov/pubmed/22805315

275. Kochlik, B.,Gerbracht, C.,Grune, T.,Weber, D. The Influence of Dietary Habits and Meat Consumption on Plasma 3-Methylhistidine-A Potential Marker for Muscle Protein Turnover. Mol Nutr Food Res. 2018. 62:e1701062 https://www.ncbi.nlm.nih.gov/pubmed/29573154

276. Kojima, N.,Kim, M.,Saito, K.,Yoshida, H.,Yoshida, Y.,Hirano, H.,Obuchi, S., Shimada, H.,Suzuki, T.,Kim, H. Lifestyle-Related Factors Contributing to Decline in Knee Extension Strength among Elderly Women: A Cross-Sectional and Longitudinal Cohort Study. PLoS One. 2015. 10:e0132523

https://www.ncbi.nlm.nih.gov/pubmed/26177292

277. Kolarzyk, E.,Ostachowska-Gasior, A.,Skop, A. The protein participation in daily diet and nutritional status of medical students in Krakow. Rocz Akad Med Bialymst. 2005. 50 Suppl 1:39-41 https://www.ncbi.nlm.nih.gov/pubmed/16119623

278. Komatsu, F.,Kagawa, Y.,Kawabata, T.,Kaneko, Y.,Ishiguro, K. Relationship of dietary habits and obesity to oxidative stress in Palauan people: compared with Japanese and Mongolian people. Curr Aging Sci. 2009. 2:214-22 https://www.ncbi.nlm.nih.gov/pubmed/20021416

279. Komatsu, F.,Kagawa, Y.,Kawabata, T.,Kaneko, Y.,Purvee, B.,Otgon, J. Chimedregzen, U. Dietary habits of Mongolian people, and their influence on lifestyle-related diseases and early aging. Curr Aging Sci. 2008. 1:84-100 https://www.ncbi.nlm.nih.gov/pubmed/20021377

280. Komatsu, F.,Kagawa, Y.,Sakuma, M.,Kawabata, T.,Kaneko, Y..Otgontuya, D., Chimedregzen, U.,Narantuya, L.,Purvee, B. Investigation of oxidative stress and dietary habits in Mongolian people, compared to Japanese people. Nutr Metab (Lond). 2006. 3:21 https://www.ncbi.nlm.nih.gov/pubmed/16759377

281. Koopman, R. Dietary protein and exercise training in ageing. Proc Nutr Soc. 2011 70:104-13 https://www.ncbi.nlm.nih.gov/pubmed/21092364

282. Koppes, L. L.,Boon, N.,Nooyens, A. C.,van Mechelen, W.,Saris, W. H. Macronutrient distribution over a period of 23 years in relation to energy intake and body fatness. Br J Nutr. 2009 101:108-15 https://www.ncbi.nlm.nih.gov/pubmed/18466652

283. Koster, A.,Penninx, B. W. J. H.,Newman, A. B.,Visser, M.,Van Gool, C. H.,Harris, T B.,Van Eijk, J. Th M.,Kempen, G. I. J. M.,Brach, J. S.,Simonsick, E. M.,Houston, D. K.,Tylavsky, F. A.,Rubin, S. M.,Kritchevsky, S. B. Lifestyle factors and incident mobility limitation in obese and non-obese older adults. Obesity. 2007. 15:31223132

http://www.embase.com/search/results?subaction=viewrecord\&from=export\&id=L3 51256676

284. Kukuljan, S.,Nowson, C. A.,Sanders, K.,Daly, R. M. Effects of resistance exercise and fortified milk on skeletal muscle mass, muscle size, and functional performance in middle-aged and older men: an 18-mo randomized controlled trial. J Appl Physio (1985). 2009. 107:1864-73 https://www.ncbi.nlm.nih.gov/pubmed/19850735 
285. Kuwabara, A.,Tsugawa, N.,Kondo, H.,Ao, M.,Fujiwara, H.,Hosokawa, N., Matsumoto, S., Tanaka, K.,Nakano, T. Associations between serum 25-

$\mathrm{X}$

hydroxyvitamin D3 level and skeletal muscle mass and lower limb muscle strength

in Japanese middle-aged subjects. Osteoporos Sarcopenia. 2017. 3:53-58 https://www.ncbi.nlm.nih.gov/pubmed/30775503

286. Kwon, J.,Yoshida, Y.,Yoshida, H.,Kim, H.,Suzuki, T.,Lee, Y. Effects of a combined physical training and nutrition intervention on physical performance and healthrelated quality of life in prefrail older women living in the community: A randomized controlled trial. Journal of the American Medical Directors Association. 2015. 16:263.e1-263.e8

http://www.embase.com/search/results?subaction=viewrecord\&from=export\&id=L6 02005847

http://dx.doi.org/10.1016/j.jamda.2014.12.005

287. Kynde, I.,Bjornsbo, K. S.,Tetens, I.,Heitmann, B. L. Dietary carbohydrates and change in physical performance of elderly Europeans: Survey in Europe on Nutrition and the Elderly, a Concerted Action (SENECA) 1993 and 1999. Public Health Nutr. 2010. 13:1186-90 https://www.ncbi.nlm.nih.gov/pubmed/19807934

288. Laclaustra, M.,Rodriguez-Artalejo, F.,Guallar-Castillon, P.,Banegas, J. R.,Graciani, A.,Garcia-Esquinas, E.,Lopez-Garcia, E. The inflammatory potential of diet is related to incident frailty and slow walking in older adults. Clin Nutr. 2019 \#volume\#:\#pages\# https://www.ncbi.nlm.nih.gov/pubmed/30737049

289. LaFountain, R. A.,Miller, V. J.,Barnhart, E. C.,Hyde, P. N.,Crabtree, C. D., McSwiney, F. T.,Beeler, M. K.,Buga, A.,Sapper, T. N.,Short, J. A.,Bowling, M. L., Kraemer, W. J.,Simonetti, O. P.,Maresh, C. M.,Volek, J. S. Extended Ketogenic Diet and Physical Training Intervention in Military Personnel. Mil Med. 2019. \#volume\#:\#pages\# https://www.ncbi.nlm.nih.gov/pubmed/30877806

290. Lan, T. Y.,Hou, S. M. Chen, C. Y.Chang, W. C.,Lin, J.,Lin, C. C. Liu, W. J.,Shih, T. F.,Tai, T. Y. Risk factors for hip fracture in older adults: a case-control study in Taiwan. Osteoporos Int. 2010. 21:773-84 https://www.ncbi.nlm.nih.gov/pubmed/19597907

291. Landi, F.,Calvani, R.,Tosato, M.,Martone, A. M.,Picca, A.,Ortolani, E.,Savera, G. Salini, S.,Ramaschi, M.,Bernabei, R.,Marzetti, E. Animal-Derived Protein Consumption Is Associated with Muscle Mass and Strength in CommunityDwellers: Results from the Milan EXPO Survey. J Nutr Health Aging. 2017. 21:1050-1056 https://www.ncbi.nlm.nih.gov/pubmed/29083447

292. Lauque, S.,Arnaud-Battandier, F.,Mansourian, R.,Guigoz, Y.,Paintin, M. Nourhashemi, F.,Vellas, B. Protein-energy oral supplementation in malnourished nursing-home residents. A controlled trial. Age Ageing. 2000. 29:51-6 https://www.ncbi.nlm.nih.gov/pubmed/10690696

293. Lee, J. S.,Kritchevsky, S. B.,Tylavsky, F. A.,Harris, T.,Everhart, J.,Simonsick, E. M., $\quad$ X Rubin, S. M.,Newman, A. B. Weight-loss intention in the well-functioning, community-dwelling elderly: associations with diet quality, physical activity, and weight change. Am J Clin Nutr. 2004. 80:466-74 https://www.ncbi.nlm.nih.gov/pubmed/15277172

294. Lee, K. S.,Lee, J. K.,Yeun, Y. R. Effects of a 10-Day Intensive Health Promotion Program Combining Diet and Physical Activity on Body Composition, Physical Fitness, and Blood Factors of Young Adults: A Randomized Pilot Study. Med Sci Monit. 2017. 23:1759-1767 https://www.ncbi.nlm.nih.gov/pubmed/28399076 
295. Leidy, H. J.,Carnell, N. S.,Mattes, R. D.,Campbell, W. W. Higher protein intake preserves lean mass and satiety with weight loss in pre-obese and obese women. Obesity (Silver Spring). 2007. 15:421-9

https://www.ncbi.nlm.nih.gov/pubmed/17299116

296. Leite, M. L.,Nicolosi, A. Lifestyle correlates of anthropometric estimates of body adiposity in an Italian middle-aged and elderly population: a covariance analysis. Int J Obes (Lond). 2006. 30:926-34 https://www.ncbi.nlm.nih.gov/pubmed/16432539

297. Lemieux, F. C.,Filion, M. E.,Barbat-Artigas, S.,Karelis, A. D.,Aubertin-Leheudre, M. Relationship between different protein intake recommendations with muscle mass and muscle strength. Climacteric. 2014. 17:294-300 https://www.ncbi.nlm.nih.gov/pubmed/23931598

298. Leon-Munoz, L. M.,Garcia-Esquinas, E.,Lopez-Garcia, E.,Banegas, J. R., Rodriguez-Artalejo, F. Major dietary patterns and risk of frailty in older adults: a prospective cohort study. BMC Med. 2015. 13:11 https://www.ncbi.nlm.nih.gov/pubmed/25601152

299. Leon-Munoz, L. M.,Guallar-Castillon, P.,Lopez-Garcia, E.,Rodriguez-Artalejo, F. Mediterranean diet and risk of frailty in community-dwelling older adults. J Am Med Dir Assoc. 2014. 15:899-903 https://www.ncbi.nlm.nih.gov/pubmed/25127502

300. Liberman, K.,Njemini, R.,Luiking, Y.,Forti, L. N.,Verlaan, S.,Bauer, J. M.,Memelink, R.,Brandt, K.,Donini, L. M.,Maggio, M.,Mets, T.,Wijers, S. L. J.,Sieber, C.,

Cederholm, T.,Bautmans, I. Thirteen weeks of supplementation of vitamin D and leucine-enriched whey protein nutritional supplement attenuates chronic low-grade inflammation in sarcopenic older adults: the PROVIDE study. Aging Clin Exp Res. 2019. 31:845-854 https://www.ncbi.nlm.nih.gov/pubmed/31049877

301. Lim, S. H.,Fan, S. H.,Say, Y. H. Plasma total antioxidant capacity (TAC) in obese Malaysian subjects. Malays J Nutr. 2012. 18:345-54 https://www.ncbi.nlm.nih.gov/pubmed/24568075

302. Lima-Silva, A. E.,Pires, F. O.,Bertuzzi, R.,Silva-Cavalcante, M. D.,Oliveira, R. S. Kiss, M. A.,Bishop, D. Effects of a low- or a high-carbohydrate diet on performance, energy system contribution, and metabolic responses during supramaximal exercise. Appl Physiol Nutr Metab. 2013. 38:928-34 https://www.ncbi.nlm.nih.gov/pubmed/23905657 lactovegetarians compared with omnivores. Atherosclerosis. 2001. 158:247-51 https://www.ncbi.nlm.nih.gov/pubmed/11500198

304. Lindhardt, T.,Nielsen, M. H. Older patients' use of technology for a post-discharge nutritional intervention - A mixed-methods feasibility study. International Journal of Medical Informatics. 2017. 97:312-321

$\mathrm{http}: / / \mathrm{www} . \mathrm{embase} . \mathrm{com} / \mathrm{search} /$ results? subaction=viewrecord\&from=export\&id=L6 13206355 http://dx.doi.org/10.1016/j.ijmedinf.2016.10.017

305. Lo, Y. C.,Wahlqvist, M. L.,Huang, Y. C.,Chuang, S. Y.,Wang, C. F. Lee, M. S. Medical costs of a low skeletal muscle mass are modulated by dietary diversity and physical activity in community-dwelling older Taiwanese: a longitudinal study. Int $J$ Behav Nutr Phys Act. 2017. 14:31 https://www.ncbi.nlm.nih.gov/pubmed/2828865 
Lockwood, C. M.,Moon, J. R.,Tobkin, S. E.,Walter, A. A.,Smith, A. E.,Dalbo, V. J.,

$\mathrm{X}$

Cramer, J. T.,Stout, J. R. Minimal nutrition intervention with high-protein/low-

carbohydrate and low-fat, nutrient-dense food supplement improves body

composition and exercise benefits in overweight adults: A randomized controlled

trial. Nutrition and Metabolism. 2008. 5:\#pages\#

$\mathrm{http}: / / \mathrm{ww} w . e m b a s e . c o m / s e a r c h /$ results? subaction=viewrecord\&from=export\&id=L3

51679532

http://dx.doi.org/10.1186/1743-7075-5-11

307. Loenneke, J. P.,Balapur, A.,Thrower, A. D.,Syler, G.,Timlin, M.,Pujol, T. J. Short report: Relationship between quality protein, lean mass and bone health. Ann Nutr Metab. 2010. 57:219-20 https://www.ncbi.nlm.nih.gov/pubmed/21124023

308. Loenneke, J. P.,Pujol, T. J. Sarcopenia: An emphasis on occlusion training and dietary protein. Hippokratia. 2011. 15:132-7

https://www.ncbi.nlm.nih.gov/pubmed/22110294

309. Longland, T. M.,Oikawa, S. Y.,Mitchell, C. J.,Devries, M. C.,Phillips, S. M. Higher compared with lower dietary protein during an energy deficit combined with intense exercise promotes greater lean mass gain and fat mass loss: a randomized trial. Am J Clin Nutr. 2016. 103:738-46 https://www.ncbi.nlm.nih.gov/pubmed/26817506

310. Lopez-Fontana, C. M.,Sanchez-Villegas, A.,Martinez-Gonzalez, M. A.,Martinez, J. A. Daily physical activity and macronutrient distribution of low-calorie diets jointly affect body fat reduction in obese women. Appl Physiol Nutr Metab. 2009. 34:595602 https://www.ncbi.nlm.nih.gov/pubmed/19767793

311. Loprinzi, P. D.,Frith, E. Effects of Sedentary Behavior, Physical Activity, Frequency of Protein Consumption, Lower Extremity Strength and Lean Mass on All-Cause Mortality. J Lifestyle Med. 2018. 8:8-15 https://www.ncbi.nlm.nih.gov/pubmed/29581955

312. Lord, C., Chaput, J. P.,Aubertin-Leheudre, M.,Labonte, M.,Dionne, I. J. Dietary animal protein intake: association with muscle mass index in older women. J Nut Health Aging. 2007. 11:383-7 https://www.ncbi.nlm.nih.gov/pubmed/17657359

313. Ma, W.,Hagan, K. A.,Heianza, Y.,Sun, Q.,Rimm, E. B.,Qi, L. Adult height, dietary patterns, and healthy aging. Am J Clin Nutr. 2017. 106:589-596 https://www.ncbi.nlm.nih.gov/pubmed/28592610

314. Macchi, C.,Molino-Lova, R.,Polcaro, P.,Guarducci, L.,Lauretani, F.,Cecchi, F., Bandinelli, S.,Guralnik, J. M.,Ferrucci, L. Higher circulating levels of uric acid are prospectively associated with better muscle function in older persons. Mech Ageing Dev. 2008. 129:522-7 https://www.ncbi.nlm.nih.gov/pubmed/18534661

315. Machado-Fragua, M. D.,Struijk, E. A.,Ballesteros, J. M.,Ortola, R.,RodriguezArtalejo, F.,Lopez-Garcia, E. Habitual coffee consumption and risk of falls in 2 Artalejo, F., Lopez-Garcia, E. Habitual coffee consumption and risk of fall
European cohorts of older adults. Am J Clin Nutr. 2019. 109:1431-1438 https://www.ncbi.nlm.nih.gov/pubmed/31005970

316. Malafarina, V.,Serra Rexach, J. A.,Masanes, F.,Cruz-Jentoft, A. J. Effects of highprotein, high-calorie oral nutritional supplementation in malnourished older people protein, high-calorie oral nutritional supplementation in malnourished older people
in nursing homes: An observational, multi-center, prospective study (PROT-eGER). Protocol and baseline population characteristics. Maturitas. 2019. 126:73-79 http://www.embase.com/search/results?subaction=viewrecord\&from=export\&id=L2 002021384

http://dx.doi.org/10.1016/j.maturitas.2019.05.009 
317. Malenfant, P.,Tremblay, A.,Doucet, E.,Imbeault, P.,Simoneau, J. A.,Joanisse, D. R.

$\mathrm{X}$
Elevated intramyocellular lipid concentration in obese subjects is not reduced after diet and exercise training. Am J Physiol Endocrinol Metab. 2001. 280:E632-9 https://www.ncbi.nlm.nih.gov/pubmed/11254471

318. Mamerow, M. M.,Mettler, J. A.,English, K. L.,Casperson, S. L.,Arentson-Lantz, E., Sheffield-Moore, M.,Layman, D. K.,Paddon-Jones, D. Dietary protein distribution positively influences 24-h muscle protein synthesis in healthy adults. J Nutr. 2014. 144:876-80 https://www.ncbi.nlm.nih.gov/pubmed/24477298

319. Mangano, K. M.,Sahni, S.,Kiel, D. P.,Tucker, K. L.,Dufour, A. B.,Hannan, M. T. Dietary protein is associated with musculoskeletal health independently of dietary pattern: the Framingham Third Generation Study. Am J Clin Nutr. 2017. 105:714722 https://www.ncbi.nlm.nih.gov/pubmed/28179224

320. Manios, Y.,Moschonis, G.,Koutsikas, K.,Papoutsou, S.,Petraki, I.,Bellou, E., Naoumi, A., Kostea, S., Tanagra, S. Changes in body composition following a dietary and lifestyle intervention trial: the postmenopausal health study. Maturitas. 2009. 62:58-65 https://www.ncbi.nlm.nih.gov/pubmed/19118956

321. Manjunath, H.,Prabha, V.,Venkatesh, D.,Taklikar, R. H. Electromyogram, hand grip strength and time to fatigue in matched samples of vegetarians and nonvegetarians. National Journal of Physiology, Pharmacy and Pharmacology. 2017 7:808-811

http://www.embase.com/search/results?subaction=viewrecord\&from=export\&id=L6 17689511

http://dx.doi.org/10.5455/njppp.2017.7.0410907042017

322. Manning, F., Harris, K.,Duncan, R.,Walton, K.,Bracks, J.,Larby, L., Vari, L.,Jukkola, K.,Bell, J.,Chan, M.,Batterham, M. Additional feeding assistance improves the energy and protein intakes of hospitalised elderly patients. A health services evaluation. Appetite 2012 59:471-7

https://www.ncbi.nlm.nih.gov/pubmed/22735333

323. Marcos-Forniol, E.,Meco, J. F.,Corbella, E.,Formiga, F.,Pinto, X. Secondary prevention programme of ischaemic heart disease in the elderly: A randomised clinical trial. Eur J Prev Cardiol. 2018. 25:278-286 https://www.ncbi.nlm.nih.gov/pubmed/29164902

324. Marighela, T. F., Genaro Pde, S.,Pinheiro, M. M.,Szejnfeld, V. L.,Kayser, C. Risk factors for body composition abnormalities in systemic sclerosis. Clin Rheumatol. 2013. 32:1037-44 https://www.ncbi.nlm.nih.gov/pubmed/23549639

325. Marques, E. A.,Moreira, P.,Wanderley, F.,Pizarro, A. N.,Leao-Rosas, J. P.,Mota, J., Carvalho, J. Appendicular fat mass is positively associated with femoral neck bone mineral density in older women. Menopause. 2012. 19:311-8 https://www.ncbi.nlm.nih.gov/pubmed/22027941

326. Marques, E.,Wanderley, F.,Goncalves, D.,Coelho, M., Viana, J.,Moreira, P.,Mota, J Carvalho, J. A comparison of resistance and aerobic exercise training on physical ability, bone mineral density and osteoprotegerin in older women. Journal of bone and mineral research.. 2010. 25:S87

https://www.cochranelibrary.com/central/doi/10.1002/central/CN-01060907/full

327. Martin, H.,Aihie Sayer, A., Jameson, K.,Syddall, H.,Dennison, E. M.,Cooper, C. Robinson, S. Does diet influence physical performance in community-dwelling older people? Findings from the Hertfordshire Cohort Study. Age Ageing. 2011. 40:181-6 https://www.ncbi.nlm.nih.gov/pubmed/21239409 
Martin, I. S. M.,Barato, V. P.,Oliva, S. L.,Rodriguez, M.,Yurrita, L. C.,Cabanas, M. J.

C.,Rojo, S. S.,de la Calle, L.,Diaz, E. A.,Santos, Y. Q.,Pascual, P. E.,Nieto, M. V.,

Vilar, E. G. Body Composition, Dietary, and Gustatory Function Assessment in

People With Alzheimer's Disease. Am J Alzheimers Dis Other Demen. 2018.

33:508-515 https://www.ncbi.nlm.nih.gov/pubmed/29916258

329. Martinez-Rodriguez, A.,Roche, E. Effect of satiety on body composition and anxiety

in university athletes: cohort study. Nutr Hosp. 2017. 34:396-401

https://www.ncbi.nlm.nih.gov/pubmed/28421796

330. McClure, R., Villani, A. Greater adherence to a Mediterranean Diet is associated

with better gait speed in older adults with type 2 diabetes mellitus. Clinical Nutrition ESPEN. 2019. \#volume\#:\#pages\#

http://www.embase.com/search/results?subaction=viewrecord\&from=export\&id=L2 002056577

http://dx.doi.org/10.1016/j.clnesp.2019.05.009

331. McDonald, C.,Bauer, J.,Capra, S.,Coll, J. The muscle mass, omega-3, diet, exercise and lifestyle (MODEL) study - a randomised controlled trial for women who have completed breast cancer treatment. BMC Cancer. 2014. 14:264

https://www.ncbi.nlm.nih.gov/pubmed/24739260

332. McLean, R. R.,Mangano, K. M.,Hannan, M. T.,Kiel, D. P.,Sahni, S. Dietary Protein Intake Is Protective Against Loss of Grip Strength Among Older Adults in the Framingham Offspring Cohort. J Gerontol A Biol Sci Med Sci. 2016. 71:356-61 https://www.ncbi.nlm.nih.gov/pubmed/26525088

333. McSwiney, F. T.,Wardrop, B.,Hyde, P. N.,Lafountain, R. A.,Volek, J. S.,Doyle, L. Keto-adaptation enhances exercise performance and body composition responses to training in endurance athletes. Metabolism: Clinical and Experimental. 2018.

81:25-34

$\mathrm{http}: / / \mathrm{www} . \mathrm{embase} . \mathrm{com} / \mathrm{search} /$ results?subaction=viewrecord\&from=export\&id=L6

19470973

http://dx.doi.org/10.1016/j.metabol.2017.10.010

334. Mears, M.,Tussing-Humphreys, L.,Cerwinske, L., Tangney, C.,Hughes, S. L.,

Fitzgibbons, M.,Gomez-Perez, S. Associations between Alternate Healthy Eating Index-2010, Body Composition, Osteoarthritis Severity, and Interleukin-6 in Older Overweight and Obese African American Females with Self-Reported Osteoarthritis. Nutrients. 2018. 11:\#pages\# https://www.ncbi.nlm.nih.gov/pubmed/30583501

335. Meirelles, C.,Candido, T.,Gomes, P. S. Effects of short-term very low-carbohydrate or conventional diet on strength performance. J Sports Med Phys Fitness. 2010. 50:189-95 https://www.ncbi.nlm.nih.gov/pubmed/20585297

336. Mendonca, N.,Granic, A.,Mathers, J. C.,Hill, T. R.,Siervo, M.,Adamson, A. J. Jagger, $C$. Prevalence and determinants of low protein intake in very old adults: insights from the Newcastle 85+ Study. Eur J Nutr. 2018. 57:2713-2722 https://www.ncbi.nlm.nih.gov/pubmed/28948346

337. Meng, X.,Zhu, K.,Devine, A.,Kerr, D. A.,Binns, C. W.,Prince, R. L. A 5-year cohort study of the effects of high protein intake on lean mass and BMC in elderly postmenopausal women. J Bone Miner Res. 2009. 24:1827-34 https://www.ncbi.nlm.nih.gov/pubmed/19419320 
338. Merra, G.,Gratteri, S.,De Lorenzo, A.,Barrucco, S.,Perrone, M. A.,Avolio, E., Bernardini, S.,Marchetti, M.,Di Renzo, L. Effects of very-low-calorie diet on body $\mathrm{X}$ composition, metabolic state, and genes expression: a randomized double-blind placebo-controlled trial. Eur Rev Med Pharmacol Sci. 2017. 21:329-345 https://www.ncbi.nlm.nih.gov/pubmed/28165552

339. Merra, G.,Miranda, R.,Barrucco, S., Gualtieri, P.,Mazza, M.,Moriconi, E.,Marchetti, M.,Chang, T. F.,De Lorenzo, A.,Di Renzo, L. Very-low-calorie ketogenic diet with aminoacid supplement versus very low restricted-calorie diet for preserving muscle mass during weight loss: a pilot double-blind study. Eur Rev Med Pharmacol Sci. 2016. 20:2613-21 https://www.ncbi.nlm.nih.gov/pubmed/27383313

340. Messier, S. P.,Loeser, R. F.,Mitchell, M. N.,Valle, G.,Morgan, T. P.,Rejeski, W. J., Ettinger, W. H. Exercise and weight loss in obese older adults with knee osteoarthritis: a preliminary study. J Am Geriatr Soc. 2000. 48:1062-72 https://www.ncbi.nlm.nih.gov/pubmed/10983905

341. Michalczyk, M.,Zajac, A.,Mikolajec, K.,Zydek, G.,Langfort, J. No Modification in Blood Lipoprotein Concentration but Changes in Body Composition after 4 Weeks of Low Carbohydrate Diet (LCD) Followed by 7 Days of Carbohydrate Loading in Basketball Players. J Hum Kinet. 2018. 65:125-137 https://www.ncbi.nlm.nih.gov/pubmed/30687425

342. Mielgo-Ayuso, J.,Zourdos, M. C.,Calleja-Gonzalez, J.,Urdampilleta, A.,Ostojic, S. M. Dietary intake habits and controlled training on body composition and strength in elite female volleyball players during the season. Appl Physiol Nutr Metab. 2015. 40:827-34 https://www.ncbi.nlm.nih.gov/pubmed/26224330

343. Milaneschi, Y.,Bandinelli, S.,Corsi, A. M.,Lauretani, F.,Paolisso, G.,Dominguez, L. J.,Semba, R. D.,Tanaka, T.,Abbatecola, A. M.,Talegawkar, S. A.,Guralnik, J. M., Ferrucci, L. Mediterranean diet and mobility decline in older persons. Exp Gerontol. 2011. 46:303-8 https://www.ncbi.nlm.nih.gov/pubmed/21111801

344. Miller, G. D. Improved nutrient intake in older obese adults undergoing a structured diet and exercise intentional weight loss program. J Nutr Health Aging. 2010. 14:461-6 https://www.ncbi.nlm.nih.gov/pubmed/20617289

345. Miller, G. D.,Rejeski, W. J.,Williamson, J. D.,Morgan, T.,Sevick, M. A.,Loeser, R. F., $\quad$ X Ettinger, W. H.,Messier, S. P. The Arthritis, Diet and Activity Promotion Trial (ADAPT): design, rationale, and baseline results. Control Clin Trials. 2003. 24:46280 https://www.ncbi.nlm.nih.gov/pubmed/12865040

346. Milte, C. M.,Thorpe, M. G.,Crawford, D.,Ball, K.,McNaughton, S. A. Associations of diet quality with health-related quality of life in older Australian men and women. Exp Gerontol. 2015. 64:8-16 https://www.ncbi.nlm.nih.gov/pubmed/25639944

347. Mitchell, C. J.,Milan, A. M.,Mitchell, S. M.,Zeng, N.,Ramzan, F.,Sharma, P Knowles, S. O.,Roy, N. C.,Sjodin, A.,Wagner, K. H.,Cameron-Smith, D. The effects of dietary protein intake on appendicular lean mass and muscle function in elderly men: a 10-wk randomized controlled trial. Am J Clin Nutr. 2017. 106:1375-1383 https://www.ncbi.nlm.nih.gov/pubmed/29092886

348. Miyatake, N.,Nishikawa, H.,Morishita, A.,Kunitomi, M.,Wada, J.,Suzuki, H., Takahashi, K.,Makino, H.,Kira, S.,Fujii, M. Daily walking reduces visceral adipose tissue areas and improves insulin resistance in Japanese obese subjects. Diabetes Res Clin Pract. 2002. 58:101-7 https://www.ncbi.nlm.nih.gov/pubmed/12213351

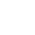


349. Mohamad, H. A., Jr.,Suzana, S.,Noor Ibrahim, M. S.,Norshafarina, S. Relationship

$\mathrm{X}$

between Appetite, Food Intake and Body Composition among Elderly Malays from

an Urban Residential Area in Kuala Lumpur, Malaysia. Malays J Nutr. 2010.

16:339-48 https://www.ncbi.nlm.nih.gov/pubmed/22691987

350. Mohammed, S. S.,Aghdassi, E.,Salit, I. E.,Avand, G.,Sherman, M.,Guindi, M., Heathcote, J. E.,Allard, J. P. HIV-positive patients with nonalcoholic fatty liver disease have a lower body mass index and are more physically active than HIVnegative patients. J Acquir Immune Defic Syndr. 2007. 45:432-8

https://www.ncbi.nlm.nih.gov/pubmed/17558337

351. Mohorko, N.,Cernelic-Bizjak, M.,Poklar-Vatovec, T.,Grom, G.,Kenig, S.,Petelin, A., Jenko-Praznikar, Z. Weight loss, improved physical performance, cognitive function, eating behavior, and metabolic profile in a 12-week ketogenic diet in obese adults. Nutr Res. 2019. 62:64-77

https://www.ncbi.nlm.nih.gov/pubmed/30803508

352. Mojtahedi, M. C.,Thorpe, M. P.,Karampinos, D. C.,Johnson, C. L.,Layman, D. K., Georgiadis, J. G.,Evans, E. M. The effects of a higher protein intake during energy restriction on changes in body composition and physical function in older women. Gerontol A Biol Sci Med Sci. 2011. 66:1218-25

https://www.ncbi.nlm.nih.gov/pubmed/21798863

353. Moon, K. T. Decreasing fall risk in older adults with nutritional intervention. American Family Physician. 2012. 86:676

http://www.embase.com/search/results?subaction=viewrecord\&from=export\&id=L3 65811917

354. Moore, D. R.,Del Bel, N. C.,Nizi, K. I.,Hartman, J. W.,Tang, J. E.,Armstrong, D., Phillips, S. M. Resistance training reduces fasted- and fed-state leucine turnover and increases dietary nitrogen retention in previously untrained young men. $J$ Nutr. 2007. 137:985-91 https://www.ncbi.nlm.nih.gov/pubmed/17374665

355. Moran, D. S.,Heled, Y.,Arbel, Y.,Israeli, E.,Finestone, A. S.,Evans, R. K., Yanovich, R. Dietary intake and stress fractures among elite male combat recruits. $J$ Int Soc Sports Nutr. 2012. 9:6 https://www.ncbi.nlm.nih.gov/pubmed/22413851

356. Moreto, F.,de Oliveira, E. P.,Manda, R. M.,Torezan, G. A.,Teixeira, O.,Michelin, E., Burini, R. C. Pathological and behavioral risk factors for higher serum C-reactive protein concentrations in free-living adults--a Brazilian community-based study. Inflammation. 2013. 36:15-25 https://www.ncbi.nlm.nih.gov/pubmed/22865000

357. Morris, M. S.,Jacques, P. F. Total protein, animal protein and physical activity in relation to muscle mass in middle-aged and older Americans. Br J Nutr. 2013. 109:1294-303 https://www.ncbi.nlm.nih.gov/pubmed/22856586

358. Moschonis, G.,Tanagra, S.,Koutsikas, K.,Nikolaidou, A.,Androutsos, O.,Manios, Y. Association between serum 25-hydroxyvitamin D levels and body composition in postmenopausal women: the postmenopausal Health Study. Menopause. 2009. 16:701-7 https://www.ncbi.nlm.nih.gov/pubmed/19276997

359. Motevalli, M. S.,Dalbo, V. J.,Attarzadeh, R. S.,Rashidlamir, A.,Tucker, P. S., Scanlan, A. T. The effect of rate of weight reduction on serum myostatin and follistatin concentrations in competitive wrestlers. Int J Sports Physiol Perform. 2015. 10:139-46 https://www.ncbi.nlm.nih.gov/pubmed/24911427

$\mathrm{x}$

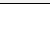

$\mathrm{x} \quad \mathrm{x}$

$\mathrm{X}$

$\mathrm{X}$

$\mathrm{X}$

X 
360. Mulla, U. Z.,Cooper, R.,Mishra, G. D.,Kuh, D.,Stephen, A. M. Adult macronutrien

$\mathrm{X}$
intake and physical capability in the MRC National Survey of Health and Development. Age Ageing. 2013. 42:81-7

https://www.ncbi.nlm.nih.gov/pubmed/22923606

361. Muller, K.,Morais, J.,Feine, J. Nutritional and anthropometric analysis of edentulous patients wearing implant overdentures or conventional dentures. Braz Dent J. 2008. 19:145-50 https://www.ncbi.nlm.nih.gov/pubmed/18568230

362. Munk, T.,Beck, A. M.,Holst, M.,Rosenbom, E.,Rasmussen, H. H.,Nielsen, M. A., Thomsen, T. Positive effect of protein-supplemented hospital food on protein intake in patients at nutritional risk: a randomised controlled trial. J Hum Nutr Diet. 2014 27:122-32 https://www.ncbi.nlm.nih.gov/pubmed/24479388

363. Murphy, C. H.,Saddler, N. I.,McGlory, C.,Devries, M. C.,Baker, S. K.,Phillips, S. M. Leucine supplementation enhances integrated myofibrillar protein synthesis in older men consuming lower and higher protein diets. Clinical nutrition (edinburgh, scotland). 2016. 35:S132

https://www.cochranelibrary.com/central/doi/10.1002/central/CN-01361469/full

364. Muscariello, E.,Nasti, G.,Siervo, M.,Di Maro, M.,Lapi, D.,D'Addio, G.,Colantuoni, A. Dietary protein intake in sarcopenic obese older women. Clin Interv Aging. 2016. 11:133-40 https://www.ncbi.nlm.nih.gov/pubmed/26917955

365. Mustafa, J.,Ellison, R. C.,Singer, M. R.,Bradlee, M. L.,Kalesan, B.,Holick, M. F Moore, L. L. Dietary Protein and Preservation of Physical Functioning Among Middle-Aged and Older Adults in the Framingham Offspring Study. Am J Epidemiol. 2018. 187:1411-1419 https://www.ncbi.nlm.nih.gov/pubmed/29590270

366. Nabuco, H. C. G., Tomeleri, C. M.,Fernandes, R. R.,Sugihara Junior, P.,Cavalcante, E. F.,Cunha, P. M.,Antunes, M.,Nunes, J. P.,Venturini, D.,Barbosa, D. S.,Burini, R. C.,Silva, A. M.,Sardinha, L. B.,Cyrino, E. S. Effect of whey protein supplementation combined with resistance training on body composition, muscular strength, functional capacity, and plasma-metabolism biomarkers in older women with sarcopenic obesity: A randomized, double-blind, placebo-controlled trial. Clinical Nutrition ESPEN. 2019. \#volume\#:\#pages\#

http://www.embase.com/search/results?subaction=viewrecord\&from=export\&id=L2 001954541

http://dx.doi.org/10.1016/j.clnesp.2019.04.007

367. Nabuco, H. C. G.,Tomeleri, C. M.,Sugihara Junior, P.,Fernandes, R. R., Cavalcante, E. F.,Venturini, D.,Barbosa, D. S.,Silva, A. M.,Sardinha, L. B.,Cyrino, E. S. Effects of pre- or post-exercise whey protein supplementation on body fat and metabolic and inflammatory profile in pre-conditioned older women: A randomized, doubleblind, placebo-controlled trial. Nutr Metab Cardiovasc Dis. 2019. 29:290-300 https://www.ncbi.nlm.nih.gov/pubmed/30639252

368. Nabuco, H. C.,Tomeleri, C. M.,Junior, P. S.,Fernandes, R. R.,Cavalcante, E. F., Nunes, J. P.,Cunha, P. F.,Dos Santos, L.,Cyrino, E. S. Effects of higher habitual protein intake on resistance-training-induced changes in body composition and muscular strength in untrained older women: a clinical trial study. Nutrition and health. 2019. \#volume\#:260106019838365

http://www.embase.com/search/results?subaction=viewrecord\&from=export\&id=L6 27046600

http://dx.doi.org/10.1177/0260106019838365 
Nagai, Y.,Kawanabe, S.,Fukuda, H.,Tanaka, Y. Changes of body composition afte replacing dietary carbohydrate with a protein supplement in overweight Japanese subjects. Journal of diabetes investigation. 2018. 9:44-

https://www.cochranelibrary.com/central/doi/10.1002/central/CN-01793199/full

370. Nahas, P. C.,Rossato, L. T.,Martins, F. M.,Souza, A. P.,de Branco, F. M. S. Carneiro, M. A. S., Teixeira, K. R. C.,Orsatti, F. L., de Oliveira, E. P. Moderate Increase in Protein Intake Promotes a Small Additional Improvement in Functional Capacity, But Not in Muscle Strength and Lean Mass Quality, in Postmenopausal Women Following Resistance Exercise: A Randomized Clinical Trial. Nutrients. 2019. 11:\#pages\# https://www.ncbi.nlm.nih.gov/pubmed/31200437

371. Nahas, P.,Rossato, L.,De Branco, F.,Martins, F.,Souza, A.,Carneiro, M.,Orsatti, F., De Oliveira, E. Changes in lean mass is correlated with changes in muscle function only in postmenopausal women who consumed higher amounts of protein during resistance training protocol. Annals of nutrition \& metabolism. 2017. 71:529https://www.cochranelibrary.com/central/doi/10.1002/central/CN-01428820/full

372. Nahhas, R. W.,Choh, A. C.,Lee, M.,Cameron Chumlea, W. M.,Duren, D. L., Siervogel, R. M.,Sherwood, R. J.,Towne, B.,Czerwinski, S. A. Bayesian longitudinal plateau model of adult grip strength. American Journal of Human Biology. 2010. 22:648-656

http://www.embase.com/search/results?subaction=viewrecord\&from=export\&id=L3 59681368

http://dx.doi.org/10.1002/ajhb.21057

373. Naidoo, R.,Coopoo, Y. The health and fitness profiles of nurses in KwaZulu-Natal. Curationis. 2007. 30:66-73 https://www.ncbi.nlm.nih.gov/pubmed/17703824

374. Nakamura, K.,Nishiwaki, T.,Ueno, K.,Yamamoto, M. Serum 25-hydroxyvitamin D levels and activities of daily living in noninstitutionalized elderly Japanese requiring care. J Bone Miner Metab. 2005. 23:488-94

https://www.ncbi.nlm.nih.gov/pubmed/16261457

375. Nascimento, M. A. D.,Gerage, A. M.,Silva, Drpd,Ribeiro, A. S.,Machado, Dgds, Pina, F. L. C., Tomeleri, C. M.,Venturini, D.,Barbosa, D. S.,Mayhew, J. L.,Cyrino, E. $S$. Effect of resistance training with different frequencies and subsequent detraining on muscle mass and appendicular lean soft tissue, IGF-1, and testosterone in older women. Eur J Sport Sci. 2019. 19:199-207 women. Eur J Sport Sci. 2019. 19:199-207
https://www.ncbi.nlm.nih.gov/pubmed/30016192

376. Nasreddine, L.,Akika, R.,Mailhac, A. Tamim, H.,Zgheib, N. K The Interaction between Genetic Polymorphisms in FTO and TCF7L2 Genes and Dietary Intake with Regard to Body Mass and Composition: An Exploratory Study. J Pers Med. 2019. 9:\#pages\# https://www.ncbi.nlm.nih.gov/pubmed/30764585

377. Nazare, J. A.,Smith, J.,Borel, A. L.,Almeras, N.,Tremblay, A.,Bergeron, J.,Poirier P.,Despres, J. P. Changes in both global diet quality and physical activity level synergistically reduce visceral adiposity in men with features of metabolic syndrome. J Nutr. 2013. 143:1074-83 https://www.ncbi.nlm.nih.gov/pubmed/23719226

378. Nct, Impact of Interventions With a Diet and Exercise on the Cardio-metabolic Status in Multi-risk Population. Https://clinicaltrials.gov/show/nct03701425. 2018. \#volume\#:\#pages\# https://www.cochranelibrary.com/central/doi/10.1002/central/CN-01663999/full

$\mathrm{X}$

X
$\mathrm{X}$

$x \quad x$

$x \quad x$

$x$


379. Nct, Mediterranean Diet, Circuit Resistance Training, Empagliflozin in Elderly With

$\mathrm{X}$
Type 2 Diabetes: a Study Protocol. Https:///linicaltrials.gov/show/nct03560375. 2018. \#volume\#:\#pages\#

https://www.cochranelibrary.com/central/doi/10.1002/central/CN-01660423/full

380. Nct, Diet and Muscle Function In Older Adults. Https://clinicaltrials.gov/show/nct02069314. 2014. \#volume\#:\#pages\# https://www.cochranelibrary.com/central/doi/10.1002/central/CN-01543860/full

381. Nct, Effects of High Protein Diet on Muscle Mass, Strength, and Physical Performance in Postmenopausal Women.

Https://clinicaltrials.gov/show/nct03652584. 2018. \#volume\#:\#pages\# https://www.cochranelibrary.com/central/doi/10.1002/central/CN-01662789/full

382. Neelemaat, F.,Bosmans, J. E.,Thijs, A.,Seidell, J. C.,van Bokhorst-de van der Schueren, M. A. E. Post-Discharge Nutritional Support in Malnourished Elderly Individuals Improves Functional Limitations. Journal of the American Medical Directors Association. 2011. 12:295-301

http://www.embase.com/search/results?subaction=viewrecord\&from=export\&id=L3

61656698

http://dx.doi.org/10.1016/j.jamda.2010.12.005

383. Negro, M.,Vandoni, M.,Ottobrini, S.,Codrons, E.,Correale, L.,Buonocore, D., Marzatico, F. Protein supplementation with low fat meat after resistance training: Effects on body composition and strength. Nutrients. 2014. 6:3040-3049

http://www.embase.com/search/results?subaction=viewrecord\&from=export\&id=L3

73710926

http://dx.doi.org/10.3390/nu6083040

384. Neville, C. E.,Young, I. S., Gilchrist, S. E.,McKinley, M. C.,Gibson, A.,Edgar, J. D., Woodside, J. V. Effect of increased fruit and vegetable consumption on physical

function and muscle strength in older adults. Age (Dordr). 2013. 35:2409-22

https://www.ncbi.nlm.nih.gov/pubmed/23543264

385. Nilsson, A.,Montiel Rojas, D.,Kadi, F. Impact of Meeting Different Guidelines for Protein Intake on Muscle Mass and Physical Function in Physically Active Older Women. Nutrients. 2018. 10:\#pages\#

https://www.ncbi.nlm.nih.gov/pubmed/30149519

386. Norton, C.,Toomey, C.,McCormack, W. G.,Francis, P.,Saunders, J.,Kerin, E., Jakeman, P. Protein Supplementation at Breakfast and Lunch for 24 Weeks beyond Habitual Intakes Increases Whole-Body Lean Tissue Mass in Healthy Older Adults. J Nutr. 2016. 146:65-9 https://www.ncbi.nlm.nih.gov/pubmed/26581685

387. Norton, C., Toomey, C.,McCormack, W.,Francis, P.,Jakeman, P. Effect of 6 month supplemental milk protein intake on lean tissue mass in healthy adults aged 50 to 70 years. Proceedings of the nutrition society. 2015. 74:\#pages\#

https://www.cochranelibrary.com/central/doi/10.1002/central/CN-01407723/full

388. Novakova, K.,Kummer, O.,Bouitbir, J.,Stoffel, S. D.,Hoerler-Koerner, U.,Bodmer,

M.,Roberts, P.,Urwyler, A.,Ehrsam, R., Krähenbühl, S. Effect of l-carnitine

supplementation on the body carnitine pool, skeletal muscle energy metabolism

and physical performance in male vegetarians. European Journal of Nutrition.

2016. 55:207-217

http://www.embase.com/search/results?subaction=viewrecord\&from=export\&id=L6

02140696

http://dx.doi.org/10.1007/s00394-015-0838-9 
389. Nowson, C.,O'Connell, S.,Mundell, N.,Grimes, C.,Dunstan, D.,Daly, R. A protein-

$\mathrm{X}$
enriched diet favourably affects cardiovascular health in elderly women undertaking progressive resistance training. Annals of nutrition \& metabolism. 2013. 63:657 https://www.cochranelibrary.com/central/doi/10.1002/central/CN-01025195/full

390. Nygard, L. K.,Mundal, I.,Dahl, L.,Rokstad, A. M. M. Nutrition and sarcopenia in frail elderly: a randomized controlled trial of the effects of marine protein hydrolysates to improve physical performance. Journal of cachexia, sarcopenia and muscle. 2017. 8:181- https://www.cochranelibrary.com/central/doi/10.1002/central/CN01361590/full

391. Ocobock, C. J. Body fat attenuates muscle mass catabolism among physically active humans in temperate and cold high altitude environments. Am J Hum Biol. 2017. 29:\#pages\# https://www.ncbi.nlm.nih.gov/pubmed/28467016

392. Ogata Medel, M.,Lozada Mellado, M.,Garcia Morales, J. M.,Hinojosa Azaola, A., Morales Herrero, N.,Llorente, L.,Alcocer Varela, J.,Pineda Juarez, J. A.,Cervantes Gaytan, R.,Castillo Martinez, L. Effect of a dynamic exercise program in combination with a mediterranean diet in weight and handgrip strength in women with rheumatoid arthritis. Clinical and experimental rheumatology. 2018. 36:S78https://www.cochranelibrary.com/central/doi/10.1002/central/CN-01605063/full

393. Oh, C., Jeon, B. H., Reid Storm, S. N.,Jho, S.,No, J. K. The most effective factors to offset sarcopenia and obesity in the older Korean: Physical activity, vitamin D, and protein intake. Nutrition. 2017. 33:169-173 https://www.ncbi.nlm.nih.gov/pubmed/27717662

394. Oh, C.,Jho, S.,No, J. K.,Kim, H. S. Body composition changes were related to nutrient intakes in elderly men but elderly women had a higher prevalence of sarcopenic obesity in a population of Korean adults. Nutr Res. 2015. 35:1-6 https://www.ncbi.nlm.nih.gov/pubmed/25524331

395. Oh, C.,No, J. K.,Kim, H. S. Dietary pattern classifications with nutrient intake and body composition changes in Korean elderly. Nutr Res Pract. 2014. 8:192-7 https://www.ncbi.nlm.nih.gov/pubmed/24741404

396. Okita, K., Takada, S.,Kinugawa, S. Very low-carbohydrate diet can effectively reduce weight without deterioration in physical fitness. Journal of the hong kong college of cardiology. 2016. 24:A32-

https://www.cochranelibrary.com/central/doi/10.1002/central/CN-01789343/full

397. Ordonez, A. M.,Madalozzo Schieferdecker, M. E.,Cestonaro, T.,Cardoso Neto, J., Ligocki Campos, A. C. Nutritional status influences the length of stay and clinical outcomes in patients hospitalized in internal medicine wards. Nutr Hosp. 2013. 28:1313-20 https://www.ncbi.nlm.nih.gov/pubmed/23889658

398. Orsatti, F. L.,Maesta, N.,de Oliveira, E. P.,Nahas Neto, J.,Burini, R. C.,Nunes, P. R. P.,Souza, A. P.,Martins, F. M.,Nahas, E. P. Adding Soy Protein to Milk Enhances the Effect of Resistance Training on Muscle Strength in Postmenopausal Women. $J$ Diet Suppl. 2018. 15:140-152 https://www.ncbi.nlm.nih.gov/pubmed/28604135

399. Ortola, R., Garcia-Esquinas, E.,Leon-Munoz, L. M.,Guallar-Castillon, P.,ValenciaMartin, J. L., Galan, I.,Rodriguez-Artalejo, F. Patterns of Alcohol Consumption and Risk of Frailty in Community-dwelling Older Adults. J Gerontol A Biol Sci Med Sci. 2016. 71:251-8 https://www.ncbi.nlm.nih.gov/pubmed/26297937

$\mathrm{X}$

X

$x$


in Metabolic Syndrome Patients Treated with Three Different Diets: A Post-Hoc

Analysis from a Randomized Controlled Clinical Trial. Nutrients. 2018. 10:\#pages\#

https://www.ncbi.nlm.nih.gov/pubmed/29958455

401. Otsuka, R.,Kato, Y.,Nishita, Y.,Tange, C.,Nakamoto, M.,Tomida, M.,Imai, T.,Ando, F.,Shimokata, H.,Suzuki, T. Dietary diversity and 14-year decline in higher-level functional capacity among middle-aged and elderly Japanese. Nutrition. 2016. 32:784-9 https://www.ncbi.nlm.nih.gov/pubmed/27068882

402. Otsuka, R., Tange, C., Tomida, M.,Nishita, Y.,Kato, Y.,Yuki, A.,Ando, F.,Shimokata, $\mathrm{H}$.,Arai, H. Dietary factors associated with the development of physical frailty in community-dwelling older adults. J Nutr Health Aging. 2019. 23:89-95 https://www.ncbi.nlm.nih.gov/pubmed/30569075

403. Ottestad, I.,Lovstad, A. T.,Gjevestad, G. O.,Hamarsland, H.,Saltyte Benth, J., Andersen, L. F.,Bye, A.,Biong, A. S., Retterstol, K.,Iversen, P. O.,Raastad, T., Ulven, S. M.,Holven, K. B. Intake of a Protein-Enriched Milk and Effects on Muscle Mass and Strength. A 12-Week Randomized Placebo Controlled Trial among Community-Dwelling Older Adults. J Nutr Health Aging. 2017. 21:1160-1169 https://www.ncbi.nlm.nih.gov/pubmed/29188875

404. Panagaria, N.,Varma, K.,Nijhawan, S.,Mathur, A.,Rai, R. R. Quality of life and nutritional status in alcohol addicts and patients with chronic liver disease. Trop Gastroenterol. 2007. 28:171-5 https://www.ncbi.nlm.nih.gov/pubmed/18416348

405. Paoli, A.,Grimaldi, K.,D'Agostino, D.,Cenci, L.,Moro, T.,Bianco, A.,Palma, A Ketogenic diet does not affect strength performance in elite artistic gymnasts. $J$ Int Soc Sports Nutr. 2012. 9:34 https://www.ncbi.nlm.nih.gov/pubmed/22835211

406. Paoli, A.,Pacelli, Q. F.,Cancellara, P.,Toniolo, L.,Moro, T.,Canato, M.,Miotti, D.,Neri, M.,Morra, A., Quadrelli, M.,Reggiani, C. Protein Supplementation Does Not Further Increase Latissimus Dorsi Muscle Fiber Hypertrophy after Eight Weeks of Resistance Training in Novice Subjects, but Partially Counteracts the Fast-to-Slow Muscle Fiber Transition. Nutrients. 2016. 8:\#pages\# https://www.ncbi.nlm.nih.gov/pubmed/27258300

407. Paoli, A.,Pacelli, Q. F.,Neri, M.,Toniolo, L.,Cancellara, P.,Canato, M.,Moro, T., Quadrelli, M.,Morra, A.,Faggian, D.,Plebani, M.,Bianco, A.,Reggiani, C. Protein supplementation increases Postexercise plasma Myostatin concentration after 8 weeks of resistance training in young physically active subjects. Journal of Medicinal Food. 2015. 18:137-143

http://www.embase.com/search/results?subaction=viewrecord\&from=export\&id=L6 01208657 http://dx.doi.org/10.1089/jmf.2014.0004

408. Park, S. Change in physical performance in older women: role of protein supplement, diet, and obesity. Journal of the american geriatrics society. 2014 62:S184- https://www.cochranelibrary.com/central/doi/10.1002/central/CN01010375/full

409. Park, S.,Na, W.,Sohn, C. Relationship between osteosarcopenic obesity and dietary inflammatory index in postmenopausal Korean women: 2009 to 2011 Korea National Health and Nutrition Examination Surveys. J Clin Biochem Nutr. 2018. 63:211-216 https://www.ncbi.nlm.nih.gov/pubmed/30487671 
Park, Y.,Choi, J. E.,Hwang, H. S. Protein supplementation improves muscle mass and physical performance in undernourished prefrail and frail elderly subjects: a randomized, double-blind, placebo-controlled trial. Am J Clin Nutr. 2018. 108:10261033 https://www.ncbi.nlm.nih.gov/pubmed/30475969

411. Parr, E. B.,Coffey, V. G.,Burke, L. M.,Phillips, S. M.,Hawley, J. A. Effect of increased dairy consumption on weight loss during 16 weeks of energy restriction and exercise training. International journal of sport nutrition and exercise metabolism. Conference: 2014 international sports and exercise nutrition conference, ISENC 2014. United kingdom. 2015. 25:1-2

https://www.cochranelibrary.com/central/doi/10.1002/central/CN-01669755/full

412. Parsons, T. J.,Papachristou, E.,Atkins, J. L.,Papacosta, O.,Ash, S.,Lennon, L. T., Whincup, P. H.,Ramsay, S. E.,Wannamethee, S. G. Physical frailty in older men: prospective associations with diet quality and patterns. Age Ageing. 2019. 48.355360 https://www.ncbi.nlm.nih.gov/pubmed/30668624

413. Parsons, T. J.,Papachristou, E.,Atkins, J. L.,Papacosta, O.,Ash, S.,Lennon, L. T. Whincup, P. H.,Ramsay, S. E.,Wannamethee, S. G. Healthier diet quality and dietary patterns are associated with lower risk of mobility limitation in older men. Eur J Nutr. 2019. 58:2335-2343 https://www.ncbi.nlm.nih.gov/pubmed/30039434

414. Pasiakos, S. M.,Cao, J. J.,Margolis, L. M.,Sauter, E. R.,Whigham, L. D.,McClung, J. P.,Rood, J. C.,Carbone, J. W.,Combs, G. F., Jr.,Young, A. J. Effects of high-protein diets on fat-free mass and muscle protein synthesis following weight loss: a randomized controlled trial. Faseb j. 2013. 27:3837-47

https://www.ncbi.nlm.nih.gov/pubmed/23739654

415. Penteado, Vsdr,Castro, C. H. M.,Pinheiro, M. M.,Santana, M.,Bertolino, S.,de Mello, M. T.,Szejnfeld, V. L. Diet, body composition, and bone mass in well-trained cyclists. J Clin Densitom. 2010. 13:43-50

https://www.ncbi.nlm.nih.gov/pubmed/19942468

416. Perala, M. M.,von Bonsdorff, M. B.,Mannisto, S.,Salonen, M. K.,Simonen, M., Kanerva, N.,Rantanen, T.,Pohjolainen, P.,Eriksson, J. G. The healthy Nordic diet predicts muscle strength 10 years later in old women, but not old men. Age Ageing 2017. 46:588-594 https://www.ncbi.nlm.nih.gov/pubmed/28338859

417. Perala, M. M.,von Bonsdorff, M.,Mannisto, S.,Salonen, M. K.,Simonen, M.,Kanerva N.,Pohjolainen, P.,Kajantie, E.,Rantanen, T.,Eriksson, J. G. A healthy Nordic diet and physical performance in old age: findings from the longitudinal Helsinki Birth Cohort Study. Br J Nutr. 2016. 115:878-86

https://www.ncbi.nlm.nih.gov/pubmed/26785760

418. Perez, L. M.,Enfedaque-Montes, M. B.,Cesari, M.,Soto-Bagaria, L.,Gual, N., Burbano, M. P.,Tarazona-Santabalbina, F. J.,Casas, R. M.,Diaz, F.,Martin, E., Gomez, A.,Orfila, F.,Inzitari, M. A Community Program of Integrated Care for Frail Older Adults: +AGIL Barcelona. J Nutr Health Aging. 2019. 23:710-716 https://www.ncbi.nlm.nih.gov/pubmed/31560028

419. Perez-Tasigchana, R. F.,Leon-Munoz, L. M.,Lopez-Garcia, E.,Banegas, J. R., Rodriguez-Artalejo, F., Guallar-Castillon, P. Mediterranean Diet and Health-Related Quality of Life in Two Cohorts of Community-Dwelling Older Adults. PLoS One.

2016. 11:e0151596 https://www.ncbi.nlm.nih.gov/pubmed/27008160 
420. Peterson, M. J. The Risk Factors of Performance-Based Early Frailty in Midlife and $\mathrm{X}$ Older Age. Gerontology and Geriatric Medicine. 2018. 4:\#pages\# http://www.embase.com/search/results?subaction=viewrecord\&from=export\&id=L6 23248860

http://dx.doi.org/10.1177/2333721418770035

421. Pétervári, E.,Soós, S.,Székely, M.,Balaskó, M. Alterations in the peptidergic regulation of energy balance in the course of aging. Current Protein and Peptide Science. 2011. 12:316-324

http://www.embase.com/search/results?subaction=viewrecord\&from=export\&id=L3 62082220

http://dx.doi.org/10.2174/138920311795906709

422. Petrofsky, J. S.,Ramseyn, K.,Patel, A.,Batt, J.,Prowse, M.,Bains, G. S.,Gunda, S., Sharma, A. The effect of a diet and exercise program with a mini medicine ball on cardiovascular fitness, weight loss, and strength. Journal of Applied Research. 2008. 8:116-129

http://www.embase.com/search/results?subaction=viewrecord\&from=export\&id=L3 52138191

423. Phinney, S. D. Ketogenic diets and physical performance. Nutr Metab (Lond). 2004 1:2 https://www.ncbi.nlm.nih.gov/pubmed/15507148

424. Pilgrim, A. L.,Baylis, D.,Jameson, K. A.,Cooper, C.,Sayer, A. A.,Robinson, S. M., Roberts, H. C. Measuring appetite with the simplified nutritional appetite

questionnaire identifies hospitalised older people at risk of worse health outcomes. Journal of Nutrition, Health and Aging. 2015. \#volume\#:\#pages\#

$\mathrm{http}: / / \mathrm{www}$. embase.com/search/results?subaction=viewrecord\&from=export\&id=L6 04329381

http://dx.doi.org/10.1007/s12603-015-0533-9

425. Pilis, K.,Pilis, A.,Stec, K.,Pilis, W.,Langfort, J.,Letkiewicz, S.,Michalski, C.,Czuba, M.,Zych, M.,Chalimoniuk, M. Three-Year Chronic Consumption of Low-

Carbohydrate Diet Impairs Exercise Performance and Has a Small Unfavorable

Effect on Lipid Profile in Middle-Aged Men. Nutrients. 2018. 10:\#pages\# https://www.ncbi.nlm.nih.gov/pubmed/30518095

426. Pilleron, S.,Ajana, S.,Jutand, M. A.,Helmer, C.,Dartigues, J. F.,Samieri, C.,Feart, C Dietary Patterns and 12-Year Risk of Frailty: Results From the Three-City Bordeaux Study. J Am Med Dir Assoc. 2017. 18:169-175 https://www.ncbi.nlm.nih.gov/pubmed/27847264

427. Pilleron, S.,Peres, K.,Jutand, M. A.,Helmer, C.,Dartigues, J. F.,Samieri, C.,Feart, C. Dietary patterns and risk of self-reported activity limitation in older adults from the Three-City Bordeaux Study. Br J Nutr. 2018. 120:549-556 https://www.ncbi.nlm.nih.gov/pubmed/29987992

428. Pineda-Juarez, J. A.,Ogata-Medel, M.,Lozada-Mellado, M.,Castillo-Martinez, L., Hinojosa-Azaola, A.,Gonzalez-Contreras, M.,Cervantes-Gaytan, R.,Garcia-Morales, J. M.,Alcocer-Varela, J.,Orea-Tejeda, A.,et al., Effect of a dynamic exercise program in combination with a mediterranean diet in strength, joint mobility and disease activity in women with rheumatoid arthritis. Arthritis \& rheumatology. 2016 68:3303-3304 https://www.cochranelibrary.com/central/doi/10.1002/central/CN01293189/full

$\mathrm{X}$


430. Pluijm, S. M. F.,Smit, J. H.,Tromp, E. A. M.,Stel, V. S.,Deeg, D. J. H.,Bouter, L. M., Lips, P. A risk profile for identifying community-dwelling elderly with a high risk of recurrent falling: Results of a 3-year prospective study. Osteoporosis International. 2006. 17:417-425

http://www.embase.com/search/results?subaction=viewrecord\&from=export\&id=L4 3461131

http://dx.doi.org/10.1007/s00198-005-0002-0

431. Porter Starr, K. N.,Orenduff, M.,McDonald, S. R.,Mulder, H.,Sloane, R.,Pieper, C. F.,Bales, C. W. Influence of Weight Reduction and Enhanced Protein Intake on

Biomarkers of Inflammation in Older Adults with Obesity. J Nutr Gerontol Geriatr.

2019. 38:33-49 https://www.ncbi.nlm.nih.gov/pubmed/30810500

432. Porter Starr, K. N.,Pieper, C. F.,Orenduff, M. C.,McDonald, S. R.,McClure, L. B.,

Zhou, R.,Payne, M. E.,Bales, C. W. Improved Function With Enhanced Protein

Intake per Meal: A Pilot Study of Weight Reduction in Frail, Obese Older Adults. J

Gerontol A Biol Sci Med Sci. 2016. 71:1369-75

https://www.ncbi.nlm.nih.gov/pubmed/26786203

433. Provan, M.,Mander, T. Mobility, exercise, nutrition and healthy ageing to avoid sarcopenia. Post Reproductive Health. 2018. 24:98-102

$\mathrm{http} / / / \mathrm{www}$. embase.com/search/results?subaction=viewrecord\&from=export\&id=L6 22705749

http://dx.doi.org/10.1177/2053369118773240

434. Pujos-Guillot, E.,Pétéra, M.,Jacquemin, J.,Centeno, D.,Lyan, B.,Montoliu, I.,Madej, D.,Pietruszka, B. Fabbri, C. Santoro, A.,Brzozowska, A.,Franceschi, C.,Comte, B. Identification of Pre-frailty Sub-Phenotypes in Elderly Using Metabolomics.

Identification of Pre-frailty Sub-Phenotypes
Frontiers in Physiology. 2019. 10:\#pages\#

$\mathrm{http}: / / \mathrm{www}$. embase.com/search/results?subaction=viewrecord\&from=export\&id=L6

27617697

http://dx.doi.org/10.3389/fphys.2018.01903

435. Puranen, T. M.,Verho, J.,Suominen, M. H. Nutritional intervention among geriatric patients after discharge-baseline findings. European geriatric medicine. 2016. 7:S153- https://www.cochranelibrary.com/central/doi/10.1002/central/CN01252722/full

436. Purcell, S.,Thornberry, R.,Elliott, S. A.,Panton, L., Ormsbee, M. J.,Vieira, E. R.,Kim, J. S.,Prado, C. M. Body Composition, Strength, and Dietary Intake of Patients with Hip or Knee Osteoarthritis. Can J Diet Pract Res. 2016. 77:98-102 https://www.ncbi.nlm.nih.gov/pubmed/26568150

437. R. B. R. sbbvp Efect of Resistance Exercise Volume on Body Composition, Physical Performance, Metabolic, Hormonal and Inflammatory Profile in Postmenopausal Pre Sarcopenic Women.

Http://www.who.int/trialsearch/trial2.aspx? Trialid=rbr-8sbbvp. 2015.

\#volume\#:\#pages\#

https://www.cochranelibrary.com/central/doi/10.1002/central/CN-01847378/full 
Racette, S. B.,Deusinger, S. S.,Strube, M. J.,Highstein, G. R.,Deusinger, R. H. Weight changes, exercise, and dietary patterns during freshman and sophomore years of college. J Am Coll Health. 2005. 53:245-51

https://www.ncbi.nlm.nih.gov/pubmed/15900988

439. Radavelli-Bagatini, S.,Zhu, K.,Lewis, J. R.,Prince, R. L. Dairy food intake, peripheral bone structure, and muscle mass in elderly ambulatory women. $J$ Bone Miner Res. 2014. 29:1691-700 https://www.ncbi.nlm.nih.gov/pubmed/24443390

440. Rahi, B.,Ajana, S.,Tabue-Teguo, M.,Dartigues, J. F.,Peres, K.,Feart, C. High adherence to a Mediterranean diet and lower risk of frailty among French older adults community-dwellers: Results from the Three-City-Bordeaux Study. Clin Nutr. 2018. 37:1293-1298 https://www.ncbi.nlm.nih.gov/pubmed/28629899

441. Rahi, B.,Morais, J. A.,Dionne, I. J.,Gaudreau, P.,Payette, H.,Shatenstein, B. The combined effects of diet quality and physical activity on maintenance of muscle strength among diabetic older adults from the NuAge cohort. Exp Gerontol. 2014. 49:40-6 https://www.ncbi.nlm.nih.gov/pubmed/24269377

442. Rahi, B.,Morais, J. A.,Gaudreau, P.,Payette, H.,Shatenstein, B. Energy and protein intakes and their association with a decline in functional capacity among diabetic older adults from the NuAge cohort. Eur J Nutr. 2016. 55:1729-39 https://www.ncbi.nlm.nih.gov/pubmed/26179475

443. Ramel, A.,Arnarson, A.,Geirsdottir, O. G.,Jonsson, P. V., Thorsdottir, I. Glomerular filtration rate after a 12-wk resistance exercise program with post-exercise protein ingestion in community dwelling elderly. Nutrition. 2013. 29:719-23 https://www.ncbi.nlm.nih.gov/pubmed/23317926

444. Rasmussen, F.,Kark, M.,Tholin, S.,Karnehed, N.,Tynelius, P. The Swedish Young Male Twins Study: a resource for longitudinal research on risk factors for obesity and cardiovascular diseases. Twin Res Hum Genet. 2006. 9:883-9 https://www.ncbi.nlm.nih.gov/pubmed/17254425

445. Ren, Z.,Huang, C.,Momma, H.,Cui, Y.,Niu, K.,Sugiyama, S.,Nanno, M.,Nagatomi, R. High Tomato and Tomato Product Consumption is Protective Against the Decline in Handgrip Strength Among Japanese Adults: The Oroshisho Study. $J$ Decline in Handgrip Strength Among Japanese Adults: The Oroshisho Study.
Epidemiol. 2018. 28:397-403 https://www.ncbi.nlm.nih.gov/pubmed/29681560

446. Rikkonen, T.,Sirola, J.,Salovaara, K.,Tuppurainen, M.,Jurvelin, J. S.,Honkanen, R., Kroger, $\mathrm{H}$. Muscle strength and body composition are clinical indicators of osteoporosis. Calcif Tissue Int. 2012. 91:131-8 https://www.ncbi.nlm.nih.gov/pubmed/22733383

447. Robinson, A. T.,Migdal, K. U.,Babcock, M. C.,Watso, J. C.,Wenner, M. M.,Stocker S. D.,Farquhar, W. B. The effects of aerobic fitness on blood pressure reactivity during controlled low and high sodium diets. FASEB journal. 2018. 32:\#pages\# https://www.cochranelibrary.com/central/doi/10.1002/central/CN-01613740/full

448. Robinson, S. M.,Jameson, K. A.,Batelaan, S. F.,Martin, H. J.,Syddall, H. E., Dennison, E. M.,Cooper, C.,Sayer, A. A. Diet and its relationship with grip strength in community-dwelling older men and women: the Hertfordshire cohort study. J Am Geriatr Soc. 2008. 56:84-90 https://www.ncbi.nlm.nih.gov/pubmed/18005355

449. Robinson, S. M.,Simmonds, S. J.,Jameson, K. A., Syddall, H. E.,Dennison, E. M., Cooper, C.,Sayer, A. A. Muscle strength in older community-dwelling men is related to type of milk feeding in infancy. J Gerontol A Biol Sci Med Sci. 2012. 67:990-6 https://www.ncbi.nlm.nih.gov/pubmed/22421706 
450. Robinson, S. M.,Westbury, L. D.,Cooper, R.,Kuh, D.,Ward, K.,Syddall, H. E.,Sayer,

$\mathrm{X}$

A. A.,Cooper, C. Adult Lifetime Diet Quality and Physical Performance in Olde Age: Findings From a British Birth Cohort. J Gerontol A Biol Sci Med Sci. 2018.

73:1532-1537 https://www.ncbi.nlm.nih.gov/pubmed/29040508

451. Robitaille, J.,Houde, A.,Lemieux, S.,Perusse, L.,Gaudet, D.,Vohl, M. C. Variants within the muscle and liver isoforms of the carnitine palmitoyltransferase I (CPT1) gene interact with fat intake to modulate indices of obesity in French-Canadians. J Mol Med (Berl). 2007. 85:129-37 https://www.ncbi.nlm.nih.gov/pubmed/17089095

452. Rocha, J. S.,Ogando, B.,Rocha, M. S.,Moreira, M. H.,Gabriel, R., Reis, V. Impact of a 12-month exercise program and hormone therapy (HT) on the body composition of postmenopausal women. Menopause (new york, N.Y.). 2012. 19:1396-

https://www.cochranelibrary.com/central/doi/10.1002/central/CN-01008526/full

453. Rodriguez-Rejon, A. I.,Ruiz-Lopez, M. D.,Artacho, R. Dietary Intake and Associated Factors in Long-Term Care Homes in Southeast Spain. Nutrients. 2019. 11:\#pages\# https://www.ncbi.nlm.nih.gov/pubmed/30691005

454. Rom, O.,Reznick, A. Z.,Keidar, Z.,Karkabi, K.,Aizenbud, D. Smoking cessationrelated weight gain--beneficial effects on muscle mass, strength and bone health. Addiction. 2015. 110:326-35 https://www.ncbi.nlm.nih.gov/pubmed/25312589

455. Roque, M.,Salva, A.,Vellas, B. Malnutrition in community-dwelling adults with dementia (Nutrialz Trial). Journal of Nutrition, Health and Aging. 2013. 17:295-299 $\mathrm{http}: / / \mathrm{www} . \mathrm{embase} . \mathrm{com} / \mathrm{search} /$ results? subaction=viewrecord\&from=export\&id=L5 2270335 http://dx.doi.org/10.1007/s12603-012-0401-9

456. Rosenberger, C.,Rechsteiner, M.,Dietsche, R.,Breidert, M. Energy and protein intake in 330 geriatric orthopaedic patients: Are the current nutrition guidelines applicable?. Clinical Nutrition ESPEN. 2019. 29:86-91

http://www.embase.com/search/results?subaction=viewrecord\&from=export\&id=L2 001382878

http://dx.doi.org/10.1016/j.clnesp.2018.11.016

457. Rosendahl, E.,Lindelöf, N.,Littbrand, H.,Yifter-Lindgren, E.,Lundin-Olsson, L., Håglin, L.,Gustafson, Y.,Nyberg, L. High-intensity functional exercise program and protein-enriched energy supplement for older persons dependent in activities of daily living: A randomised controlled trial. Australian Journal of Physiotherapy. 2006. 52:105-113

http://www.embase.com/search/results?subaction=viewrecord\&from=export\&id=L4 3971018 http://dx.doi.org/10.1016/S0004-9514(06)70045-9

458. Roth, S. M.,Zmuda, J. M.,Cauley, J. A.,Shea, P. R.,Ferrell, R. E. Vitamin D receptor genotype is associated with fat-free mass and sarcopenia in elderly men. $J$ Gerontol A Biol Sci Med Sci. 2004. 59:10-5 https://www.ncbi.nlm.nih.gov/pubmed/14718481

459. Roubenoff, R.,Walsmith, J.,Lundgren, N.,Snydman, L.,Dolnikowski, G. J.,Roberts, $\mathrm{S}$. Low physical activity reduces total energy expenditure in women with rheumatoid arthritis: implications for dietary intake recommendations. Am J Clin Nutr. 2002. 76:774-9 https://www.ncbi.nlm.nih.gov/pubmed/12324290

$\mathrm{X}$

$\mathrm{x}$

$\mathrm{X}$

$\mathrm{x} \quad \mathrm{x}$

(1)

$x$

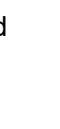

$x$



Sothier, M.,Monnet, M. F.,Ovize, M.,Bonnefoy, M.,Boesch, C.,Canet-Soulas, E. Intramyocellular lipid variations in active older men: relationship with aerobic fitness. Acta Physiol (Oxf). 2013. 207:516-23

https://www.ncbi.nlm.nih.gov/pubmed/23217190

461. Rozenek, R.,Ward, P.,Long, S.,Garhammer, J. Effects of high-calorie supplements on body composition and muscular strength following resistance training. $J$ Sports Med Phys Fitness. 2002. 42:340-7 https://www.ncbi.nlm.nih.gov/pubmed/12094125

462. Ryberg, M.,Sandberg, S.,Mellberg, C.,Stegle, O.,Lindahl, B.,Larsson, C.,Hauksson, J.,Olsson, T. A Palaeolithic-type diet causes strong tissue-specific effects on ectopic fat deposition in obese postmenopausal women. J Intern Med. 2013. 274:67-76 https://www.ncbi.nlm.nih.gov/pubmed/23414424

463. Sabia, S.,Elbaz, A.,Rouveau, N.,Brunner, E. J.,Kivimaki, M.,Singh-Manoux, A. Cumulative associations between midlife health behaviors and physical functioning in early old age: a 17-year prospective cohort study. J Am Geriatr Soc. 2014 62:1860-8 https://www.ncbi.nlm.nih.gov/pubmed/25283337

464. Sabrina, N.,Bai, C. H.,Chang, C. C.,Chien, Y. W.,Chen, J. R.,Chang, J. S. Serum Iron:Ferritin Ratio Predicts Healthy Body Composition and Reduced Risk of Severe Fatty Liver in Young Adult Women. Nutrients. 2017. 9:\#pages\# https://www.ncbi.nlm.nih.gov/pubmed/28777296

465. Saghafi-Asl, M.,Vaghef-Mehrabany, E. Comprehensive comparison of malnutrition and its associated factors between nursing home and community dwelling elderly: A case-control study from Northwestern Iran. Clin Nutr ESPEN. 2017. 21:51-58 https://www.ncbi.nlm.nih.gov/pubmed/30014869

466. Sallinen, J.,Pakarinen, A.,Ahtiainen, J.,Kraemer, W. J.,Volek, J. S.,Häkkinen, K. Relationship between diet and serum anabolic hormone responses to heavyresistance exercise in men. International Journal of Sports Medicine. 2004. 25:627 633

http://www.embase.com/search/results?subaction=viewrecord\&from=export\&id=L3 9518142 http://dx.doi.org/10.1055/s-2004-815818

467. Sallinen, J.,Pakarinen, A.,Fogelholm, M.,Alen, M.,Volek, J. S.,Kraemer, W. J., Hakkinen, K. Dietary intake, serum hormones, muscle mass and strength during strength training in 49 - 73-year-old men. Int J Sports Med. 2007. 28:1070-6 https://www.ncbi.nlm.nih.gov/pubmed/17497592

468. Sallinen, J.,Pakarinen, A.,Fogelholm, M.,Sillanpaa, E.,Alen, M.,Volek, J. S., Kraemer, W. J.,Hakkinen, K. Serum basal hormone concentrations and muscle mass in aging women: effects of strength training and diet. Int J Sport Nutr Exerc Metab. 2006. 16:316-31 https://www.ncbi.nlm.nih.gov/pubmed/16948487

469. Sammarco, R.,Marra, M.,Di Guglielmo, M. L.,Naccarato, M.,Contaldo, F., Poggiogalle, E.,Donini, L. M.,Pasanisi, F. Evaluation of Hypocaloric Diet With Protein Supplementation in Middle-Aged Sarcopenic Obese Women: A Pilot Study. Obes Facts. 2017. 10:160-167 https://www.ncbi.nlm.nih.gov/pubmed/28528340

470. Sandoval-Insausti, H.,Perez-Tasigchana, R. F.,Lopez-Garcia, E.,Garcia-Esquinas, E.,Rodriguez-Artalejo, F., Guallar-Castillon, P. Macronutrients Intake and Incident Frailty in Older Adults: A Prospective Cohort Study. J Gerontol A Biol Sci Med Sci. 2016. 71:1329-34 https://www.ncbi.nlm.nih.gov/pubmed/26946103

$X$

$\mathrm{X}$

$\mathrm{X}$

$\mathrm{X}$

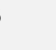

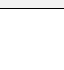

$\mathrm{x}$

$\mathrm{X}$

$x$

x $x$


Llorente, L.,Rodriguez-Guevara, G.,Castillo-Martinez, L. Prevalence of rheumatoid cachexia assessed by bioelectrical impedance vector analysis and its relation with physical function. Clin Rheumatol. 2018. 37:607-614

https://www.ncbi.nlm.nih.gov/pubmed/29119481

472. Sarti, S.,Ruggiero, E.,Coin, A.,Toffanello, E. D.,Perissinotto, E.,Miotto, F.,Pintore, G.,Inelmen, E. M.,Manzato, E.,Sergi, G. Dietary intake and physical performance in healthy elderly women: a 3-year follow-up. Exp Gerontol. 2013. 48:250-4 https://www.ncbi.nlm.nih.gov/pubmed/23063988

473. Sartor, F.,Jackson, M. J.,Squillace, C.,Shepherd, A.,Moore, J. P.,Ayer, D. E.,Kubis H. P. Adaptive metabolic response to 4 weeks of sugar-sweetened beverage consumption in healthy, lightly active individuals and chronic high glucose availability in primary human myotubes. Eur J Nutr. 2013. 52:937-48 https://www.ncbi.nlm.nih.gov/pubmed/22733000

474. Sartorio, A.,Lafortuna, C. L.,Massarini, M.,Galvani, C. Effects of different training protocols on exercise performance during a short-term body weight reduction programme in severely obese patients. Eat Weight Disord. 2003. 8:36-43 https://www.ncbi.nlm.nih.gov/pubmed/12762623

475. Sasaki, H.,Ishibashi, A., Tsuchiya, Y.,Shimura, N.,Kurihara, T.,Ebi, K.,Goto, K. A 3day high-fat/low-carbohydrate diet does not alter exercise-induced growth hormone response in healthy males. Growth Horm IGF Res. 2015. 25:304-11 https://www.ncbi.nlm.nih.gov/pubmed/26387476

476. Sawyer, J. C.,Wood, R. J.,Davidson, P. W.,Collins, S. M.,Matthews, T. D., Gregory, S. M.,Paolone, V. J. Effects of a short-term carbohydrate-restricted diet on strength and power performance. J Strength Cond Res. 2013. 27:2255-62 https://www.ncbi.nlm.nih.gov/pubmed/23774282

477. Sawyer, J. C.,Wood, R. J.,Davidson, P. W.,Collins, S. M.,Matthews, T. D., Gregory, S. M.,Paolone, V. J. Effects of a short-term carbohydrate restricted diet on strength and power performance. \#journal\#. 2013. 27:2255-2262

http://www.embase.com/search/results?subaction=viewrecord\&from=export\&id=L1 369877351

http://dx.doi.org/10.1519/JSC.0b013e31827da314

478. Schacht, S. R.,Lind, M. V.,Mertz, K. H.,Bulow, J.,Bechshoft, R.,Hojfeldt, G., Schucany, A.,Hjulmand, M.,Sidoli, C.,Andersen, S. B.,Jensen, M.,Reitelseder, S., Holm, L. Tetens, I. Development of a Mobility Diet Score (MDS) and Associations With Bone Mineral Density and Muscle Function in Older Adults. Front Nutr. 2019. 6:114 https://www.ncbi.nlm.nih.gov/pubmed/31552255

479. Schilling, B. K.,Stone, M. H.,Utter, A.,Kearney, J. T.,Johnson, M.,Coglianese, R., Smith, L.,O'Bryant, H. S.,Fry, A. C.,Starks, M.,Keith, R.,Stone, M. E. Creatine supplementation and health variables: a retrospective study. Med Sci Sports Exerc. 2001. 33:183-8 https://www.ncbi.nlm.nih.gov/pubmed/11224803

480. Schilp, J.,Kruizenga, H. M.,Wijnhoven, H. A.,van Binsbergen, J. J.,Visser, M. Effects of a dietetic treatment in older, undernourished, community-dwelling individuals in primary care: a randomized controlled trial. European journal of nutrition. 2013. 52:1939-1948

https://www.cochranelibrary.com/central/doi/10.1002/central/CN-00961710/full 
Schmid, A.,Weiss, M.,Heseker, H. Recording the nutrient intake of nursing home

$\mathrm{X}$

$\mathrm{X}$

residents by food weighing method and measuring the physical activity. J Nutr

482. Schonenberger, K. A.,Reber, E.,Bally, L.,Geiser, T.,Lin, D.,Stanga, Z. Nutritional assessment in adults with cystic fibrosis. Nutrition. 2019. 67-68:110518 https://www.ncbi.nlm.nih.gov/pubmed/31473521

483. Schrager, M. A.,Hilton, J.,Gould, R.,Kelly, V. E. Effects of blueberry supplementation on measures of functional mobility in older adults. Appl Physio Nutr Metab. 2015. 40:543-9 https://www.ncbi.nlm.nih.gov/pubmed/25909473

484. Schrijvers, J. K.,McNaughton, S. A.,Beck, K. L.,Kruger, R. Exploring the Dietary Patterns of Young New Zealand Women and Associations with BMl and Body Fat. Nutrients. 2016. 8:\#pages\# https://www.ncbi.nlm.nih.gov/pubmed/27472358

485. Schumacher, A.,Peersen, K., Sommervoll, L., Seljeflot, I.,Arnesen, H., Otterstad, J. E. Physical performance is associated with markers of vascular inflammation in patients with coronary heart disease. Eur J Cardiovasc Prev Rehabil. 2006. 13:356$62 \mathrm{https}: / /$ www.ncbi.nlm.nih.gov/pubmed/16926664

486. Scott, D.,Blizzard, L.,Fell, J.,Giles, G.,Jones, G. Associations between dietary nutrient intake and muscle mass and strength in community-dwelling older adults: the Tasmanian Older Adult Cohort Study. J Am Geriatr Soc. 2010. 58:2129-34 https://www.ncbi.nlm.nih.gov/pubmed/21054294

487. Seino, S.,Sumi, K.,Narita, M.,Yokoyama, Y.,Ashida, K.,Kitamura, A.,Shinkai, S. Effects of Low-Dose Dairy Protein Plus Micronutrient Supplementation during Resistance Exercise on Muscle Mass and Physical Performance in Older Adults: a Randomized, Controlled Trial. Journal of nutrition, health \& aging. 2018. 22:59-67 https://www.cochranelibrary.com/central/doi/10.1002/central/CN-01340380/full

488. Semba, R. D.,Gonzalez-Freire, M.,Tanaka, T.,Biancotto, A.,Zhang, P.,Shardell, M., Moaddel, R.,Ferrucci, L. Elevated Plasma Growth and Differentiation Factor-15 is Associated with Slower Gait Speed and Lower Physical Performance in Healthy Community-Dwelling Adults. J Gerontol A Biol Sci Med Sci. 2019

\#volume\#:\#pages\# https://www.ncbi.nlm.nih.gov/pubmed/30874790

489. Seo, M. H.,Kim, M. K.,Park, S. E.,Rhee, E. J.,Park, C. Y.,Lee, W. Y.,Baek, K. H., Song, K. H.,Kang, M. I.,Oh, K. W. The association between daily calcium intake and sarcopenia in older, non-obese Korean adults: the fourth Korea National Health and Nutrition Examination Survey (KNHANES IV) 2009. Endocr J. 2013. 60:679-86 https://www.ncbi.nlm.nih.gov/pubmed/23357977

490. Shahar, D. R.,Houston, D. K.,Hue, T. F.,Lee, J. S.,Sahyoun, N. R., Tylavsky, F. A., Geva, D.,Vardi, H.,Harris, T. B. Adherence to mediterranean diet and decline in walking speed over 8 years in community-dwelling older adults. J Am Geriatr Soc. 2012. 60:1881-8 https://www.ncbi.nlm.nih.gov/pubmed/23035758

491. Shahar, S.,Vanoh, D.,Mat Ludin, A. F.,Singh, D. K. A.,Hamid, T. A. Factors associated with poor socioeconomic status among Malaysian older adults: an analysis according to urban and rural settings. BMC Public Health. 2019. 19:549 https://www.ncbi.nlm.nih.gov/pubmed/31196023

492. Sharkey, J. R. Diet and health outcomes in vulnerable populations. Ann N Y Acad Sci. 2008. 1136:210-7 https://www.ncbi.nlm.nih.gov/pubmed/18579883

$\mathrm{X}$ 
493. Sharkey, J. R.,Branch, L. G. Gender difference in physical performance, body $90 \mathrm{https} / / /$ www.ncbi.nlm.nih.gov/pubmed/15778170

494. Sharkey, J. R.,Branch, L. G.,Giuliani, C.,Zohoori, M.,Haines, P. S. Nutrient intake and BMI as predictors of severity of ADL disability over 1 year in homebound elders. J Nutr Health Aging. 2004. 8:131-9

https://www.ncbi.nlm.nih.gov/pubmed/15129297

495. Sharkey, J. R., Giuliani, C.,Haines, P. S.,Branch, L. G.,Busby-Whitehead, J., Zohoori, N. Summary measure of dietary musculoskeletal nutrient (calcium, vitamin $\mathrm{D}$, magnesium, and phosphorus) intakes is associated with lower-extremity physica performance in homebound elderly men and women. Am J Clin Nutr. 2003. 77:84756 https://www.ncbi.nlm.nih.gov/pubmed/12663282

496. Shatenstein, B., Gauvin, L.,Keller, H.,Richard, L., Gaudreau, P., Giroux, F.,Jabbour, M.,Morais, J. A.,Payette, H. Individual and collective factors predicting change in diet quality over 3 years in a subset of older men and women from the NuAge cohort. Eur J Nutr. 2016. 55:1671-81

https://www.ncbi.nlm.nih.gov/pubmed/26169872

497. Shaw, S. C.,Parsons, C. M.,Fuggle, N. R.,Edwards, M. H.,Robinson, S. M., Dennison, E. M.,Cooper, C.,Ward, K. A. Diet Quality and Bone Measurements Using HRpQCT and pQCT in Older Community-Dwelling Adults from the Hertfordshire Cohort Study. Calcified Tissue International. 2018. 103:494-500 http://www.embase.com/search/results?subaction=viewrecord\&from=export\&id=L6 22665736

http://dx.doi.org/10.1007/s00223-018-0445-x

498. Shea, M. K.,Loeser, R. F.,McAlindon, T. E.,Houston, D. K.,Kritchevsky, S. B.,Booth, S. L. Association of Vitamin K Status Combined With Vitamin D Status and LowerExtremity Function: A Prospective Analysis of Two Knee Osteoarthritis Cohorts. Arthritis Care Res (Hoboken). 2018. 70:1150-1159 https://www.ncbi.nlm.nih.gov/pubmed/29045002

499. Shea, M.,Loeser, R. F.,Kritchevsky, S. B.,Houston, D. K.,McAlindon, T. E.,Booth, S $\mathrm{L}$. Is there a synergistic role for vitamin $\mathrm{K}$ and vitamin $\mathrm{D}$ in lower extremity function related to knee osteoarthritis? The osteoarthritis initiative and health, aging and body composition studies. Osteoarthritis and cartilage. Conference: 2017 osteoarthritis research society international, OARSI world congress. United states. 2017. 25:S185-S186

https://www.cochranelibrary.com/central/doi/10.1002/central/CN-01470379/full

500. Sheu, W. H. H.,Lee, W. J.,Chang, R. L.,Chen, Y. T. Plasma tumor necrosis factor alpha levels and insulin sensitivity in hypertensive subjects. Clin Exp Hypertens. 2000. 22:595-606 https://www.ncbi.nlm.nih.gov/pubmed/10972164

501. Shiina, Y.,Matsumoto, N.,Okamura, D.,Takahashi, Y.,Kijima, Y.,Fukuda, T., Kawamatsu, N.,Nishihata, Y.,Komiyama, N.,Niwa, K. Sarcopenia in adults with congenital heart disease: Nutritional status, dietary intake, and resistance training. J Cardiol. 2019. 74:84-89 https://www.ncbi.nlm.nih.gov/pubmed/30713126

502. Shimoda, T.,Suzuki, T.,Takahashi, N.,Tsutsumi, K.,Samukawa, M.,Yoshimachi, S. Goto, T.,Enomoto, H.,Kise, N.,Ogasawara, K.,Yoshimura, S. Nutritional Status and Body Composition of Independently Living Older Adults in a Snowy Region of Japan. Gerontol Geriatr Med. 2017. 3:2333721417706854 https://www.ncbi.nlm.nih.gov/pubmed/28516130 
503. Shumilov, D.,Heymsfield, S. B.,Redman, L. M.,Smith, S. R.,Bray, G. A.,Kalluri, K

$\mathrm{X}$

Shumilov, D.,Heymsfield, S. B.,Redman, L. M.,Smith, S. R.,Bray, G. A.,Kalluri, K.,
Dey, J. New compartment model analysis of lean-mass and fat-mass growth with

overfeeding. Nutrition. 2016. 32:590-600

https://www.ncbi.nlm.nih.gov/pubmed/26740258

504. Singh, N.,Graves, J.,Taylor, P. D.,MacAllister, R. J.,Singer, D. R. Effects of a

'healthy' diet and of acute and long-term vitamin $C$ on vascular function in healthy

older subjects. Cardiovasc Res. 2002. 56:118-25

https://www.ncbi.nlm.nih.gov/pubmed/12237172

505. Smee, D. J.,Pumpa, K.,Falchi, M.,Lithander, F. E. The relationship between diet quality and falls risk, physical function and body composition in older adults. Journal of Nutrition, Health and Aging. 2015. 19:1037-1042

http://www.embase.com/search/results?subaction=viewrecord\&from=export\&id=L6

07582380

http://dx.doi.org/10.1007/s12603-015-0666-x 506. Smith, A.,Gray, J. Considering the benefits of egg consumption for older people at $\quad X$

risk of sarcopenia. Br J Community Nurs. 2016. 21:305-9

https://www.ncbi.nlm.nih.gov/pubmed/27270199

507. Smith, G. I.,Yoshino, J.,Kelly, S. C.,Reeds, D. N.,Okunade, A.,Patterson, B. W., Klein, S.,Mittendorfer, B. High-Protein Intake during Weight Loss Therapy

Eliminates the Weight-Loss-Induced Improvement in Insulin Action in Obese

Postmenopausal Women. Cell Rep. 2016. 17:849-861

https://www.ncbi.nlm.nih.gov/pubmed/27732859

508. Smoliner, C.,Norman, K., Scheufele, R.,Hartig, W.,Pirlich, M.,Lochs, H. Effects of food fortification on nutritional and functional status in frail elderly nursing home residents at risk of malnutrition. Nutrition (burbank, los angeles county, calif.). 2008

24:1139-1144 https://www.cochranelibrary.com/central/doi/10.1002/central/CN00665676/full

509. Snijders, T.,Verdijk, L. B.,McKay, B. R.,Smeets, J. S.,van Kranenburg, J.,Groen, B. B.,Parise, G.,Greenhaff, P.,van Loon, L. J. Acute dietary protein intake restriction is associated with changes in myostatin expression after a single bout of resistance exercise in healthy young men. $J$ Nutr. 2014. 144:137-45

https://www.ncbi.nlm.nih.gov/pubmed/24306214

510. Son, J. W.,Lee, S. S.,Kim, S. R.,Yoo, S. J.,Cha, B. Y.,Son, H. Y.,Cho, N. H. Low muscle mass and risk of type 2 diabetes in middle-aged and older adults: findings from the KoGES. Diabetologia. 2017. 60:865-872 https://www.ncbi.nlm.nih.gov/pubmed/28102434

511. Song, M. Y.,Ruts, E.,Kim, J.,Janumala, I.,Heymsfield, S.,Gallagher, D. Sarcopenia and increased adipose tissue infiltration of muscle in elderly African American women. Am J Clin Nutr. 2004. 79:874-80

https://www.ncbi.nlm.nih.gov/pubmed/15113728

512. Stathakos, D.,Pratsinis, H.,Zachos, I.,Vlahaki, I.,Gianakopoulou, A.,Zianni, D., Kletsas, D. Greek centenarians: assessment of functional health status and lifestyle characteristics. Exp Gerontol. 2005. 40:512-8

https://www.ncbi.nlm.nih.gov/pubmed/15935588

$\mathrm{X}$ 
513. Stefler, D.,Hu, Y.,Malyutina, S.,Pajak, A.,Kubinova, R.,Peasey, A.,Pikhart, H.,

Rodriguez-Artalejo, F.,Bobak, M. Mediterranean diet and physical functioning

trajectories in Eastern Europe: Findings from the HAPIEE study. PLOS ONE. 2018.

13:\#pages\#

http://www.embase.com/search/results?subaction=viewrecord\&from=export\&id=L6

22981445

http://dx.doi.org/10.1371/journal.pone.0200460

514. Stookey, J. D.,Adair, L. S.,Popkin, B. M. Do protein and energy intakes explain long-term changes in body composition?. J Nutr Health Aging. 2005. 9:5-17

https://www.ncbi.nlm.nih.gov/pubmed/15750660

515. Stookey, J. D.,Adair, L.,Stevens, J.,Popkin, B. M. Patterns of long-term change in body composition are associated with diet, activity, income and urban residence

among older adults in China. J Nutr. 2001. 131:2433s-40s

https://www.ncbi.nlm.nih.gov/pubmed/11533290

516. Stow, R.,Ives, N.,Smith, C.,Rick, C.,Rushton, A. A cluster randomised feasibility trial evaluating nutritional interventions in the treatment of malnutrition in care home adult residents. Trials. 2015. 16:433

https://www.ncbi.nlm.nih.gov/pubmed/26416253

517. Straight, C. R.,Dorfman, L. R.,Cottell, K. E.,Krol, J. M.,Lofgren, I. E.,Delmonico, M. $\mathrm{J}$. Effects of resistance training and dietary changes on physical function and body composition in overweight and obese older adults. J Phys Act Health. 2012. 9:87583 https://www.ncbi.nlm.nih.gov/pubmed/21952180

518. Strandberg, E.,Edholm, P.,Ponsot, E.,Wahlin-Larsson, B.,Hellmen, E.,Nilsson, A. Engfeldt, P.,Cederholm, T.,Riserus, U.,Kadi, F. Influence of combined resistance training and healthy diet on muscle mass in healthy elderly women: a randomized controlled trial. J Appl Physiol (1985). 2015. 119:918-25

https://www.ncbi.nlm.nih.gov/pubmed/26338453

519. Strandberg, E.,Ponsot, E.,Piehl-Aulin, K.,Falk, G.,Kadi, F. Resistance Training Alone or Combined With N-3 PUFA-Rich Diet in Older Women: Effects on Muscle Fiber Hypertrophy. The journals of gerontology. Series A, Biological sciences and medical sciences. 2019. 74:489-494

http://www.embase.com/search/results?subaction=viewrecord\&from=export\&id=L6 26804282

http://dx.doi.org/10.1093/gerona/gly130

520. Straznicky, N. E.,Eikelis, N.,Nestel, P. J.,Dixon, J. B.,Dawood, T.,Grima, M. T.,Sari, C. I.,Schlaich, M. P.,Esler, M. D.,Tilbrook, A. J.,Lambert, G. W.,Lambert, E. A.

Baseline sympathetic nervous system activity predicts dietary weight loss in obese metabolic syndrome subjects. J Clin Endocrinol Metab. 2012. 97:605-13 https://www.ncbi.nlm.nih.gov/pubmed/22090279

521. Struijk, E. A.,Guallar-Castillon, P.,Rodriguez-Artalejo, F.,Lopez-Garcia, E.

Mediterranean Dietary Patterns and Impaired Physical Function in Older Adults. $J$

Gerontol A Biol Sci Med Sci. 2018. 73:333-339

https://www.ncbi.nlm.nih.gov/pubmed/28329809

$\mathrm{X}$

X $\mathrm{X}$

X


522. Sturgeon, K. M.,Dean, L. T.,Heroux, M.,Kane, J.,Bauer, T.,Palmer, E.,Long, J.,

$\mathrm{X}$

Lynch, S.,Jacobs, L.,Sarwer, D. B.,Leonard, M. B.,Schmitz, K. Commercially

available lifestyle modification program: randomized controlled trial addressing

heart and bone health in BRCA1/2+ breast cancer survivors after risk-reducing

salpingo-oophorectomy. J Cancer Surviv. 2017. 11:246-255

https://www.ncbi.nlm.nih.gov/pubmed/27873046

523. Sugihara Junior, P.,Ribeiro, A. S.,Nabuco, H. C. G.,Fernandes, R. R., Tomeleri, C. M.,Cunha, P. M.,Venturini, D.,Barbosa, D. S.,Schoenfeld, B. J.,Cyrino, E. S. Effects

of Whey Protein Supplementation Associated With Resistance Training on

Muscular Strength, Hypertrophy, and Muscle Quality in Preconditioned Older

Women. Int J Sport Nutr Exerc Metab. 2018. 28:528-535

https://www.ncbi.nlm.nih.gov/pubmed/29252039

524. Suh, Y.,Kang, H.,Kim, M.,Kim, S. Effect of weight reduction on Korean high protein diet in obese women. Obesity reviews. 2014. 15:229

https://www.cochranelibrary.com/central/doi/10.1002/central/CN-01041831/full

525. Tai, K.,Visvanathan, R.,Hammond, A. J.,Wishart, J. M.,Horowitz, M.,Chapman, Fasting ghrelin is related to skeletal muscle mass in healthy adults. Eur $\mathrm{J}$ Nutr. 2009. 48:176-83 https://www.ncbi.nlm.nih.gov/pubmed/19198916

526. Talegawkar, S. A.,Bandinelli, S.,Bandeen-Roche, K.,Chen, P.,Milaneschi, Y., Tanaka, T.,Semba, R. D., Guralnik, J. M.,Ferrucci, L. A higher adherence to a Mediterranean-style diet is inversely associated with the development of frailty in community-dwelling elderly men and women. J Nutr. 2012. 142:2161-6 https://www.ncbi.nlm.nih.gov/pubmed/23096005

527. Ten Haaf, D. S. M. T.,De Regt, M. F.,Visser, M.,Witteman, B. J. M.,De Vries, J. H. M.,Eijsvogels, T. M. H.,Hopman, M. T. E. Insufficient protein intake is highly prevalent among physically active elderly. Journal of Nutrition, Health and Aging 2018. 22:1112-1114

http://www.embase.com/search/results?subaction=viewrecord\&from=export\&id=L6 26914117

http://dx.doi.org/10.1007/s12603-018-1075-8

528. Ten Haaf, D. S. M.,Eijsvogels, T. M. H.,Bongers, Ccwg,Horstman, A. M. H.,

Timmers, S., de Groot, Lcpgm, Hopman, M. T. E. Protein supplementation improves

lean body mass in physically active older adults: a randomized placebo-controlled

trial. J Cachexia Sarcopenia Muscle. 2019. 10:298-310

https://www.ncbi.nlm.nih.gov/pubmed/30848096

529. Tepper, S.,Alter Sivashensky, A.,Rivkah Shahar, D.,Geva, D.,Cukierman-Yaffe, T.

The Association between Mediterranean Diet and the Risk of Falls and Physical

Function Indices in Older Type 2 Diabetic People Varies by Age. Nutrients. 2018

10:\#pages\# https://www.ncbi.nlm.nih.gov/pubmed/29899221

530. Ter Borg, S., de Groot, L. C.,Mijnarends, D. M.,de Vries, J. H.,Verlaan, S.,Meijboom,

S., Luiking, Y. C.,Schols, J. M. Differences in Nutrient Intake and Biochemical

Nutrient Status Between Sarcopenic and Nonsarcopenic Older Adults-Results From

the Maastricht Sarcopenia Study. J Am Med Dir Assoc. 2016. 17:393-401

https://www.ncbi.nlm.nih.gov/pubmed/26825685 
531. Terp, R.,Jacobsen, K. O.,Kannegaard, P.,Larsen, A. M.,Madsen, O. R.,Noiesen, E. A multidisciplinary nutritional intervention program across sectors improved the nutritional status and self-rated health in geriatric patients at nutritional risk-a randomized controlled trial. Clinical nutrition (edinburgh, scotland). 2016. 35:S55 https://www.cochranelibrary.com/central/doi/10.1002/central/CN-01361499/full

532. Thalacker-Mercer, A. E.,Fleet, J. C.,Craig, B. A.,Campbell, W. W. The skeletal muscle transcript profile reflects accommodative responses to inadequate protein

intake in younger and older males. J Nutr Biochem. 2010. 21:1076-82 https://www.ncbi.nlm.nih.gov/pubmed/20149619

533. Thalacker-Mercer, A. E.,Petrella, J. K.,Bamman, M. M. Does habitual dietary intake influence myofiber hypertrophy in response to resistance training? A cluster analysis. Appl Physiol Nutr Metab. 2009. 34:632-9

https://www.ncbi.nlm.nih.gov/pubmed/19767798

534. Thompson, B. J.,Ryan, E. D.,Sobolewski, E. J.,Smith-Ryan, A. E. Dietary protein intake is associated with maximal and explosive strength of the leg flexors in young and older blue collar workers. Nutr Res. 2015. 35:280-6 https://www.ncbi.nlm.nih.gov/pubmed/25753919

535. Tieland, M.,Borgonjen-Van den Berg, K. J.,Van Loon, L. J.,de Groot, L. C. Dietary Protein Intake in Dutch Elderly People: A Focus on Protein Sources. Nutrients. 2015. 7:9697-706 https://www.ncbi.nlm.nih.gov/pubmed/26610565

536. Tieland, M.,Borgonjen-Van den Berg, K. J.,van Loon, L. J.,de Groot, L. C. Dietary protein intake in community-dwelling, frail, and institutionalized elderly people: scope for improvement. Eur J Nutr. 2012. 51:173-9 https://www.ncbi.nlm.nih.gov/pubmed/21562887

537. Tieland, M.,van de Rest, O.,Dirks, M. L.,van der Zwaluw, N.,Mensink, M.,van Loon L. J.,de Groot, L. C. Protein supplementation improves physical performance in frail elderly people: a randomized, double-blind, placebo-controlled trial. J Am Med Dir Assoc. 2012. 13:720-6 https://www.ncbi.nlm.nih.gov/pubmed/22889730

538. Tieland, M.,Van De Rest, O.,Verdijk, L.,De Groot, L.,Van Loon, L. The effects of protein supplementation during prolonged resistance type exercise training on muscle mass and function in frail elderly. Clinical nutrition, supplement.. 2011. 6:111 https://www.cochranelibrary.com/central/doi/10.1002/central/CN01034622/full

539. Tomey, K. M.,Sowers, M. R.,Crandall, C.,Johnston, J.,Jannausch, M.,Yosef, M. Dietary intake related to prevalent functional limitations in midlife women. $A m$ Epidemiol. 2008. 167:935-43 https://www.ncbi.nlm.nih.gov/pubmed/18250080

540. Tomlinson, D. J.,Erskine, R. M.,Morse, C. I.,Onambele, G. L. Body Fat Percentage, Body Mass Index, Fat Mass Index and the Ageing Bone: Their Singular and Combined Roles Linked to Physical Activity and Diet. Nutrients. 2019. 11:\#pages\# https://www.ncbi.nlm.nih.gov/pubmed/30669348

541. Tomlinson, D. J.Erskine, R. M.,Morse, C. I.,Onambele, G. L. Impact of AboveAverage Proanabolic Nutrients Is Overridden by High Protein and Energy Intake in the Muscle-Tendon Unit Characteristics of Middle- to Older-Aged Adults. J Nutr. 2018. 148:1776-1785 https://www.ncbi.nlm.nih.gov/pubmed/30247586

$\mathrm{X}$

$x$

(n) \\ (1)
}

(1)

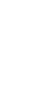

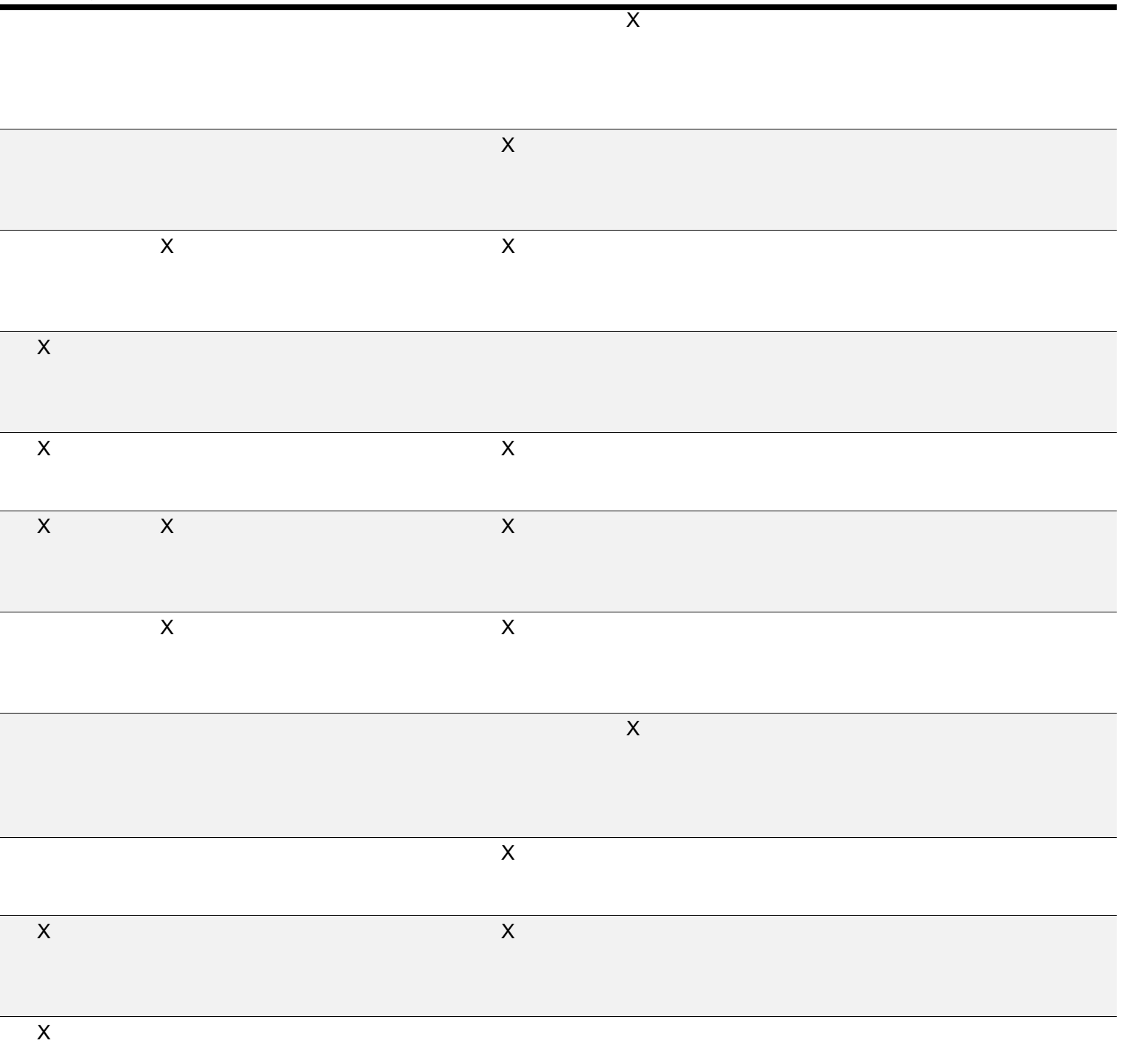

$\mathrm{X}$


542. Torres, S. J.,Robinson, S.,Orellana, L.,O'Connell, S. L., Grimes, C. A.,Mundell, N

$\mathrm{X}$

$\mathrm{X}$

L.,Dunstan, D. W.,Nowson, C. A.,Daly, R. M. Effects of progressive resistance
training combined with a protein-enriched lean red meat diet on health-related

quality of life in elderly women: secondary analysis of a 4-month cluster randomised

controlled trial. Br J Nutr. 2017. 117:1550-1559

https://www.ncbi.nlm.nih.gov/pubmed/28721837

543. Trajanoska, K.,Schoufour, J. D.,Darweesh, S. K.,Benz, E.,Medina-Gomez, C., Alferink, L. J.,Lahousse, L.,Brusselle, G.,Stricker, B.,Darwish Murad, S.,Zillikens, M. C.,Uitterlinden, A. G.,Ikram, M. A.,Franco, O. H.,Rivadeneira, F. Sarcopenia and Its Clinical Correlates in the General Population: The Rotterdam Study.J Bone Miner Res. 2018. 33:1209-1218 https://www.ncbi.nlm.nih.gov/pubmed/29502340

544. Trappe, S.,Creer, A.,Slivka, D.,Minchev, K.,Trappe, T. Single muscle fiber function with concurrent exercise or nutrition countermeasures during 60 days of bed rest in women. J Appl Physiol (1985). 2007. 103:1242-50 https://www.ncbi.nlm.nih.gov/pubmed/17641219

545. Trappe, T. A.,Burd, N. A.,Louis, E. S.,Lee, G. A., Trappe, S. W. Influence of concurrent exercise or nutrition countermeasures on thigh and calf muscle size and function during 60 days of bed rest in women. Acta Physiol (Oxf). 2007. 191:147-59 https://www.ncbi.nlm.nih.gov/pubmed/17655736

546. Trejo, A., Tarrats, R. M.,Alonso, M. E.,Boll, M. C.,Ochoa, A., Velasquez, L. Assessment of the nutrition status of patients with Huntington's disease. Nutrition. 2004. 20:192-6 https://www.ncbi.nlm.nih.gov/pubmed/14962685

547. Trevisan, C.,Veronese, N.,Maggi, S.,Baggio, G.,Toffanello, E. D.,Zambon, S. Sartori, L.,Musacchio, E.,Perissinotto, E.,Crepaldi, G.,Manzato, E.,Sergi, G. Factors Influencing Transitions Between Frailty States in Elderly Adults: The Progetto Veneto Anziani Longitudinal Study. Journal of the American Geriatrics Society. 2017. 65:179-184

http://www embase com/search/results?subaction=viewrecord\&from=export\&id=L6 13714272 http://dx.doi.org/10.1111/jgs.14515

548. Treyzon, L.,Chen, S.,Hong, K., Yan, E.,Carpenter, C. L.,Thames, G.,Bowerman, S. Wang, H. J.,Elashoff, R.,Li, Z. A controlled trial of protein enrichment of meal replacements for weight reduction with retention of lean body mass. Nutr J. 2008 7:23 https://www.ncbi.nlm.nih.gov/pubmed/18752682

549. Trierweiler, H.,Kisielewicz, G.,Hoffmann Jonasson, T.,Rasmussen Petterle, R. Aguiar Moreira, C.,Zeghbi Cochenski Borba, V. Sarcopenia: a chronic complication of type 2 diabetes mellitus. Diabetol Metab Syndr. 2018. 10:25 https://www.ncbi.nlm.nih.gov/pubmed/29632617

550. Tylner, S.,Cederholm, T.,Faxen-Irving, G. Effects on Weight, Blood Lipids, Serum Fatty Acid Profile and Coagulation by an Energy-Dense Formula to Older Care Residents: A Randomized Controlled Crossover Trial. J Am Med Dir Assoc. 2016. 17:275.e5-11 https://www.ncbi.nlm.nih.gov/pubmed/26810442

551. Tyrovolas, S.,Bountziouka, V.,Papairakleous, N.,Zeimbekis, A.,Anastassiou, F Gotsis, E.,Metallinos, G.,Polychronopoulos, E.,Lionis, C.,Panagiotakos, D. Adherence to the Mediterranean diet is associated with lower prevalence of obesity among elderly people living in Mediterranean islands: the MEDIS study. Int $J$ Food Sci Nutr. 2009. 60 Suppl 6:137-50 https://www.ncbi.nlm.nih.gov/pubmed/19672745 
552. Tyrovolas, S.,Haro, J. M.,Mariolis, A.,Piscopo, S., Valacchi, G.,Bountziouka, V., Anastasiou, F.,Zeimbekis, A., Tyrovola, D.,Foscolou, A.,Gotsis, E.,Metallinos, G., Tur, J. A.,Matalas, A.,Lionis, C.,Polychronopoulos, E.,Panagiotakos, D. Skeletal muscle mass and body fat in relation to successful ageing of older adults: The multi-national MEDIS study. Arch Gerontol Geriatr. 2016. 66:95-101 https://www.ncbi.nlm.nih.gov/pubmed/27266673

553. Urbain, P.,Strom, L.,Morawski, L.,Wehrle, A.,Deibert, P.,Bertz, H. Impact of a 6week non-energy-restricted ketogenic diet on physical fitness, body composition and biochemical parameters in healthy adults. Nutr Metab (Lond). 2017. 14:17 https://www.ncbi.nlm.nih.gov/pubmed/28239404

554. Uritani, D.,Matsumoto, D.,Asano, Y.,Yoshizaki, K.,Nishida, Y.,Shima, M. Effects of regular exercise and nutritional guidance on body composition, blood pressure, muscle strength and health-related quality of life in community-dwelling Japanese women. Obes Res Clin Pract. 2013. 7:e155-e163 https://www.ncbi.nlm.nih.gov/pubmed/24331777

555. van Dongen, E. J. I.,Haveman-Nies, A.,Wezenbeek, N. L. W.,Dorhout, B. G.,Doets, E. L.,de Groot, Lcpgm Effect, process, and economic evaluation of a combined resistance exercise and diet intervention (ProMuscle in Practice) for communitydwelling older adults: design and methods of a randomised controlled trial. BMC dwelling older adults: design and methods of a randomised controlled trial. B
Public Health. 2018. 18:877 https://www.ncbi.nlm.nih.gov/pubmed/30005654

556. Van Proeyen, K.,Szlufcik, K.,Nielens, H.,Deldicque, L.,Van Dyck, R.,Ramaekers, M.,Hespel, P. High-fat diet overrules the effects of training on fiber-specific intramyocellular lipid utilization during exercise. J Appl Physiol (1985). 2011. 111:108-16 https://www.ncbi.nlm.nih.gov/pubmed/21551007

557. Van Wymelbeke, V.,Brondel, L.,Bon, F.,Martin-Pfitzenmeyer, I.,Manckoundia, P. An innovative brioche enriched in protein and energy improves the nutritional status of usual breakfast: FARINE+ project. Clin Nutr ESPEN. 2016. 15:93-100 https://www.ncbi.nlm.nih.gov/pubmed/28531791

558. Van Zant, R. S.,Conway, J. M.,Seale, J. L. A moderate carbohydrate and fat diet does not impair strength performance in moderately trained males. Journal of Sports Medicine and Physical Fitness. 2002. 42:31-37 http://www.embase.com/search/results?subaction=viewrecord\&from=export\&id=L3 4285877

559. Vanacore, D.,Messina, G. Lama, S.,Bitti, G. Ambrosio, P. Tenore, G.,Messina, A Monda, V.,Zappavigna, S.,Boccellino, M.,Novellino, E.,Monda, M.,Stiuso, P. Effect of restriction vegan diet's on muscle mass, oxidative status, and myocytes of restriction vegan diet's on muscle mass, oxidative status, and https://www.ncbi.nlm.nih.gov/pubmed/29319158

560. Vargas, S.,Romance, R. Petro, J. L.,Bonilla, D A Galancho, I. Espinar, S.,Kreider R. B.,Benitez-Porres, J. Efficacy of ketogenic diet on body composition during resistance training in trained men: a randomized controlled trial. J Int Soc Sports Nutr. 2018. 15:31 https://www.ncbi.nlm.nih.gov/pubmed/29986720

561. Velho, S.,Moco, S.,Ferreira, A.,Cruz, R.,Agostinho, L.,Cabral, M. S.,Luz, G.,Lopes, F.,Teixeira, J. A.,Strecht, J.,Coelho, J. L. P.,Maio, R.,Cravo, M.,Baracos, V. E. Dietary patterns and their relationships to sarcopenia in Portuguese patients with gastrointestinal cancer: An exploratory study. Nutrition. 2019. 63-64:193-199 https://www.ncbi.nlm.nih.gov/pubmed/31029047

$\mathrm{X}$

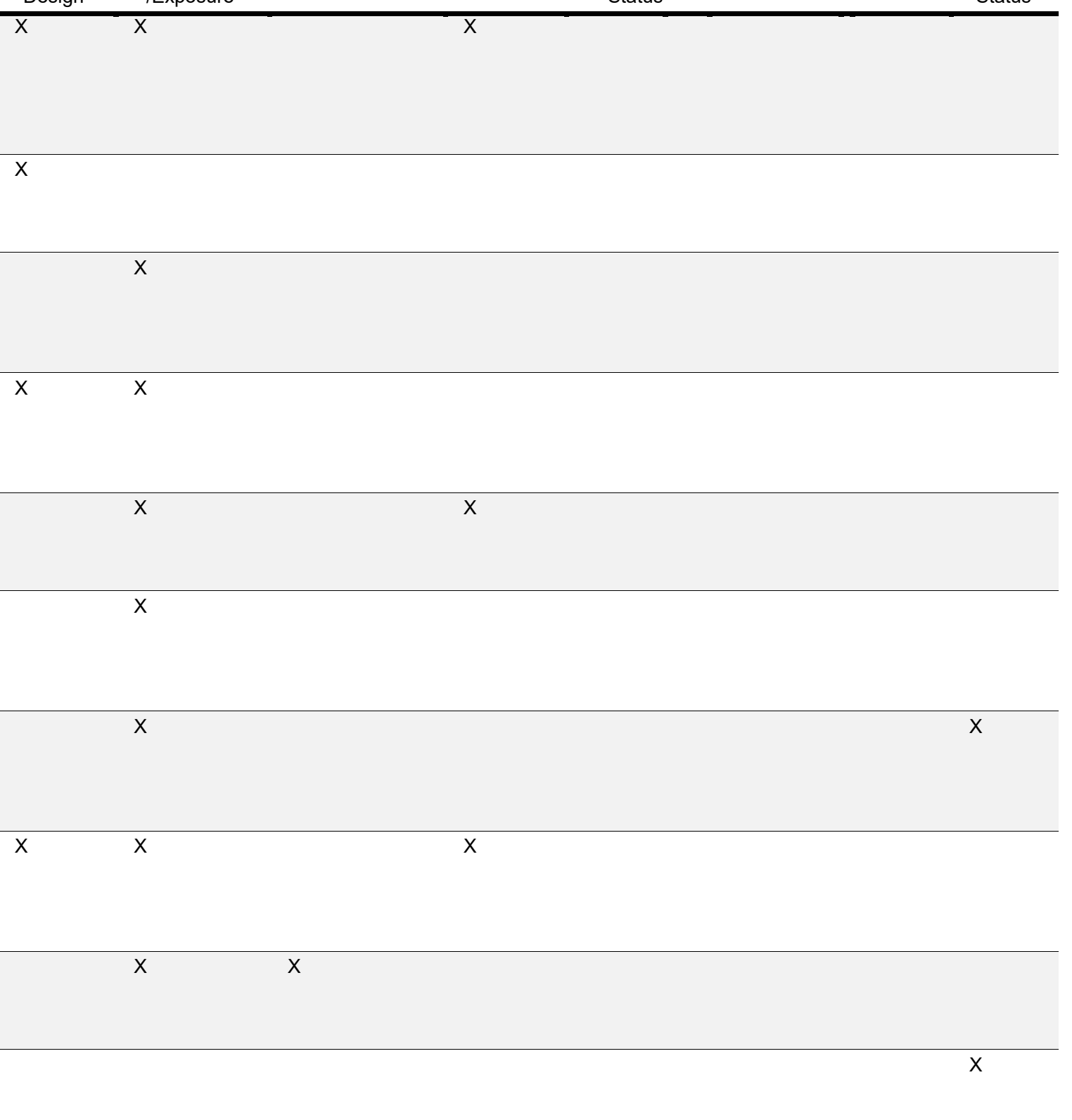

.


562. Vercambre, M. N.,Boutron-Ruault, M. C.,Ritchie, K.,Clavel-Chapelon, F.,Berr, C.

Long-term association of food and nutrient intakes with cognitive and functional

decline: a 13-year follow-up study of elderly French women. Br J Nutr. 2009.

102:419-27 https://www.ncbi.nlm.nih.gov/pubmed/19203415

563. Verhoeven, S.,Vanschoonbeek, K.,Verdijk, L. B.,Koopman, R.,Wodzig, W. K., Dendale, P.,van Loon, L. J. Long-term leucine supplementation does not increase muscle mass or strength in healthy elderly men. Am J Clin Nutr. 2009. 89:1468-75 https://www.ncbi.nlm.nih.gov/pubmed/19321567

564. Verlaan, S.,Aspray, T. J.,Bauer, J. M.,Cederholm, T.,Hemsworth, J.,Hill, T. R., McPhee, J. S.,Piasecki, M.,Seal, C.,Sieber, C. C.,Ter Borg, S.,Wijers, S. L.,Brandt, K. Nutritional status, body composition, and quality of life in community-dwelling sarcopenic and non-sarcopenic older adults: A case-control study. Clin Nutr. 2017 36:267-274 https://www.ncbi.nlm.nih.gov/pubmed/ 26689868

565. Verlaan, S.,Maier, A. B.,Bauer, J. M.,Bautmans, I.,Brandt, K.,Donini, L. M.,Maggio, M.,McMurdo, M. E. T.,Mets, T.,Seal, C.,Wijers, S. L. J.,Sieber, C.,Boirie, Y., Cederholm, T. Sufficient levels of 25 -hydroxyvitamin $D$ and protein intake required to increase muscle mass in sarcopenic older adults - The PROVIDE study. Clin Nutr. 2018. 37:551-557 https://www.ncbi.nlm.nih.gov/pubmed/28132725

566. Veronese, N.,Stubbs, B.,Maggi, S.,Notarnicola, M.,Barbagallo, M.,Firth, J., Dominguez, L. J.,Caruso, M. G. Dietary Magnesium and Incident Frailty in Older People at Risk for Knee Osteoarthritis: An Eight-Year Longitudinal Study. Nutrients. 2017. 9:\#pages\# https://www.ncbi.nlm.nih.gov/pubmed/29144404

567. Verreijen, A. M.,Engberink, M. F.,Houston, D. K.,Brouwer, I. A.,Cawthon, P. M., Newman, A. B.,Tylavsky, F. A.,Harris, T. B.,Weijs, P. J. M.,Visser, M. Dietary protein intake is not associated with 5-y change in mid-thigh muscle cross-sectional area by computed tomography in older adults: the Health, Aging, and Body Composition (Health ABC) Study. Am J Clin Nutr. 2019. 109:535-543 https://www.ncbi.nlm.nih.gov/pubmed/30850837

568. Verreijen, A. M.,Engberink, M. F.,Memelink, R. G.,van der Plas, S. E.,Visser, M., Weijs, P. J. Effect of a high protein diet and/or resistance exercise on the preservation of fat free mass during weight loss in overweight and obese older adults: a randomized controlled trial. Nutr J.2017. 16:10 https://www.ncbi.nlm.nih.gov/pubmed/28166780

569. Verreijen, A. M.,Engberink, M. F.,Memelink, R. G.,Van Der Plas, S. E.,Visser, M Weijs, P. J. Effect of a high protein diet and/or resistance exercise on preservation of fat free mass during weight loss in overweight older adults: a randomized controlled trial. Clinical nutrition (edinburgh, scotland). 2015. 34:S206https://www.cochranelibrary.com/central/doi/10.1002/central/CN-01128963/ful

570. Vieillevoye, S.,Poortmans, J. R.,Duchateau, J.,Carpentier, A. Effects of a combined essential amino acids/carbohydrate supplementation on muscle mass, architecture and maximal strength following heavy-load training. Eur J Appl Physiol. 2010. 110:479-88 https://www.ncbi.nlm.nih.gov/pubmed/20521064

571. Vila, R.,Granada, M. L.,Gutierrez, R. M.,Fernandez-Lopez, J. A.,Remesar, X., Formiguera, X.,Foz, M.,Alemany, M. Short-term effects of a hypocaloric diet on nitrogen excretion in morbid obese women. Eur J Clin Nutr. 2001. 55:186-91 https://www.ncbi.nlm.nih.gov/pubmed/11305267 $x$ 
572. Villareal, D. T.,Chode, S.,Parimi, N.,Sinacore, D. R.,Hilton, T.,Armamento-Villareal,

$\mathrm{X}$

R.,Napoli, N.,Qualls, C.,Shah, K. Weight loss, exercise, or both and physical

function in obese older adults. N Engl J Med. 2011. 364:1218-29

https://www.ncbi.nlm.nih.gov/pubmed/21449785

573. Villeneuve, N.,Pelletier-Beaumont, E.,Nazare, J. A.,Lemieux, I.,Almeras, N., Bergeron, J.,Tremblay, A.,Poirier, P.,Despres, J. P. Interrelationships between changes in anthropometric variables and computed tomography indices of abdominal fat distribution in response to a 1-year physical activity-healthy eating lifestyle modification program in abdominally obese men. Appl Physiol Nutr Metab. 2014. 39:503-11 https://www.ncbi.nlm.nih.gov/pubmed/24669993

574. Vogt, M.,Puntschart, A.,Howald, H.,Mueller, B.,Mannhart, C.,Gfeller-Tuescher, L., Mullis, P.,Hoppeler, H. Effects of dietary fat on muscle substrates, metabolism, and performance in athletes. Med Sci Sports Exerc. 2003. 35:952-60 https://www.ncbi.nlm.nih.gov/pubmed/12783043

575. Volpi, E.,Kobayashi, H.,Sheffield-Moore, M.,Mittendorfer, B.,Wolfe, R. R. Essential amino acids are primarily responsible for the amino acid stimulation of muscle protein anabolism in healthy elderly adults. Am J Clin Nutr. 2003. 78:250-8 https://www.ncbi.nlm.nih.gov/pubmed/12885705

576. Vorup, J.,Pedersen, M. T.,Brahe, L. K.,Melcher, P. S.,Alstrøm, J. M.,Bangsbo, J. Effect of small-sided team sport training and protein intake on muscle mass, physical function and markers of health in older untrained adults: A randomized trial. PLOS ONE. 2017. 12:\#pages\#

http://www.embase.com/search/results?subaction=viewrecord\&from=export\&id=L6 18693439

http://dx.doi.org/10.1371/journal.pone.0186202

577. Wakabayashi, H.,Takahashi, R.,Murakami, T. The Prevalence and Prognosis of Sarcopenic Dysphagia in Patients Who Require Dysphagia Rehabilitation. J Nut Health Aging. 2019. 23:84-88 https://www.ncbi.nlm.nih.gov/pubmed/30569074

578. Waldman, H. S.,Smith, J. W.,Lamberth, J.,Fountain, B. J.,McAllister, M. J. A 28-Day Carbohydrate-Restricted Diet Improves Markers of Cardiometabolic Health and Performance in Professional Firefighters. J Strength Cond Res. 2019. \#volume\#:\#pages\# https://www.ncbi.nlm.nih.gov/pubmed/31469768

579. Walker, J. L.,Heigenhauser, G. J. F.,Hultman, E.,Spriet, L. L. Dietary carbohydrate muscle glycogen content, and endurance performance in well-trained women. Journal of Applied Physiology. 2000. 88:2151-2158

http://www.embase.com/search/results? subaction=viewrecord\&from=export\&id=L3 0453922

580. Walrand, S.,Short, K. R.,Bigelow, M. L.,Sweatt, A. J.,Hutson, S. M.,Nair, K. S. Functional impact of high protein intake on healthy elderly people. American Journal of Physiology - Endocrinology and Metabolism. 2008. 295:E921-E928 http://www.embase.com/search/results? subaction=viewrecord\&from=export\&id=L3 http://www http://dx.doi.org/10.1152/ajpendo.90536.2008

581. Wang, C. C.,Adochio, R L.,Leitner, J W Abeyta, I M Draznin, B Cornier, M. A Acute effects of different diet compositions on skeletal muscle insulin signalling in obese individuals during caloric restriction. Metabolism. 2013. 62:595-603 https://www.ncbi.nlm.nih.gov/pubmed/23174405 
582. Wang, H. L.,Ding, T. T.,Lu, S.,Xu, Y.,Tian, J.,Hu, W. F.,Zhang, J. Y. Muscle mass loss and intermuscular lipid accumulation were associated with insulin resistance in

patients receiving hemodialysis. Chinese Medical Journal. 2013. 126:4612-4617

http://www.embase.com/search/results?subaction=viewrecord\&from=export\&id=L3

70492114

http://dx.doi.org/10.3760/cma.j.issn.0366-6999.20130976

583. Waters, D. L., Vawter, R., Qualls, C.,Chode, S.,Armamento-Villareal, R., Villareal, D. $\mathrm{T}$. Long-term maintenance of weight loss after lifestyle intervention in frail, obese

older adults. J Nutr Health Aging. 2013. 17:3-7

https://www.ncbi.nlm.nih.gov/pubmed/23299370

584. Weinheimer, E. M.,Conley, T. B.,Kobza, V. M.,Sands, L. P.,Lim, E.,Janle, E. M., Campbell, W. W. Whey protein supplementation does not affect exercise training-

induced changes in body composition and indices of metabolic syndrome in middle-

aged overweight and obese adults. Journal of Nutrition. 2012. 142:1532-1539

$\mathrm{http}: / / \mathrm{www} . \mathrm{embase} . \mathrm{com} / \mathrm{search} / \mathrm{results}$ ? subaction=viewrecord\&from=export\&id=L3 65646739

http://dx.doi.org/10.3945/jn.111.153619

585. Welch, A. A. Nutritional influences on age-related skeletal muscle loss. Proc Nutr Soc. 2014. 73:16-33 https://www.ncbi.nlm.nih.gov/pubmed/24229650

586. Welch, A. A.,MacGregor, A. J.,Minihane, A. M.,Skinner, J.,Valdes, A. A.,Spector, T. $\quad$ X

D.,Cassidy, A. Dietary fat and fatty acid profile are associated with indices of

skeletal muscle mass in women aged 18-79 years. J Nutr. 2014.144:327-34

https://www.ncbi.nlm.nih.gov/pubmed/24401817

587. Welch, A. A.,MacGregor, A. J.,Skinner, J.,Spector, T. D.,Moayyeri, A.,Cassidy, A. A $\quad X \quad X$

higher alkaline dietary load is associated with greater indexes of skeletal muscle

mass in women. Osteoporos Int 2013.24:1899-908

https://www.ncbi.nlm.nih.gov/pubmed/23152092

588. Wesseltoft-Rao, N.,Hjermstad, M. J.,Ikdahl, T.,Dajani, O.,Ulven, S. M.,Iversen, P. O.,Bye, A. Comparing two classifications of cancer cachexia and their association

with survival in patients with unresected pancreatic cancer. Nutr Cancer. 2015.

67:472-80 https://www.ncbi.nlm.nih.gov/pubmed/25710201

589. White, K. M.,Bauer, S. J.,Hartz, K. K.,Baldridge, M. Changes in body composition with yogurt consumption during resistance training in women. Int J Sport Nutr Exerc Metab. 2009. 19:18-33 https://www.ncbi.nlm.nih.gov/pubmed/19403951

590. Wilcox, G.,Strauss, B. J.,Francis, D. E.,Upton, H.,Boneh, A. Body composition in young adults with inborn errors of protein metabolism--a pilot study. $J$ Inherit Metab Dis. 2005. 28:613-26 https://www.ncbi.nlm.nih.gov/pubmed/16151892

591. Witbracht, M. G.,Laugero, K. D.,Van Loan, M. D.,Adams, S. H.,Keim, N. L. Performance on the lowa Gambling Task is related to magnitude of weight loss and salivary cortisol in a diet-induced weight loss intervention in overweight women. Physiol Behav. 2012. 106:291-7 https://www.ncbi.nlm.nih.gov/pubmed/21565212

592. Woo, J.,Chan, R.,Ong, S.,Bragt, M.,Bos, R.,Parikh, P.,de Groot, L. C. Randomized Controlled Trial of Exercise and Nutrition Supplementation on Physical and Cognitive Function in Older Chinese Adults Aged 50 Years and Older. J Am Med Dir Assoc. 2019. \#volume\#:\#pages\# https://www.ncbi.nlm.nih.gov/pubmed/31558379 
physical limitations: a 5-year follow-up study of 3,153 chinese men and women. $J$ Am Geriatr Soc. 2009. 57:2224-31 https://www.ncbi.nlm.nih.gov/pubmed/19925615

594. Wood, R. J.,Gregory, S. M.,Sawyer, J.,Milch, C. M.,Matthews, T. D.,Headley, S. A. Preservation of fat-free mass after two distinct weight loss diets with and without progressive resistance exercise. Metab Syndr Relat Disord. 2012. 10:167-74 https://www.ncbi.nlm.nih.gov/pubmed/22283635

595. Woods, J. L., luliano-Burns, S.,Walker, K. Z. Weight loss in elderly women in lowlevel care and its association with transfer to high-level care and mortality. Clin Interv Aging. 2011. 6:311-7 https://www.ncbi.nlm.nih.gov/pubmed/22267919

596. Woods, J. L., Iuliano-Burns, S.,Walker, K. Z. Immunological and nutritional factors in elderly people in low-level care and their association with mortality. Immun Ageing. 2013. 10:32 https://www.ncbi.nlm.nih.gov/pubmed/23915335

597. Wouters-Wesseling, W.,Van Hooijdonk, C.,Wagenaar, L.,Bindels, J.,de Groot, L., Van Staveren, W. The effect of a liquid nutrition supplement on body composition and physical functioning in elderly people. Clin Nutr. 2003. 22:371-7

https://www.ncbi.nlm.nih.gov/pubmed/12880604

598. Wright, C. S.,Zhou, J.,Sayer, R. D.,Kim, J. E.,Campbell, W. W. Effects of a HighProtein Diet Including Whole Eggs on Muscle Composition and Indices of Cardiometabolic Health and Systemic Inflammation in Older Adults with Overweight or Obesity: A Randomized Controlled Trial. Nutrients. 2018. 10:\#pages\# https://www.ncbi.nlm.nih.gov/pubmed/30041437

599. Wycherley, T. P.,Buckley, J. D.,Noakes, M.,Clifton, P. M.,Brinkworth, G. D. Comparison of the effects of weight loss from a high-protein versus standardprotein energy-restricted diet on strength and aerobic capacity in overweight and obese men. Eur J Nutr. 2013, 52:317-25 obese men. Eur J Nutr. 2013. 52:317-25
https://www.ncbi.nlm.nih.gov/pubmed/22406907

600. Wycherley, T. P.,Buckley, J. D.,Noakes, M.,Clifton, P. M.,Brinkworth, G. D. Longterm effects of a very low-carbohydrate weight loss diet on exercise capacity and tolerance in overweight and obese adults. J Am Coll Nutr. 2014. 33:267-73 https://www.ncbi.nlm.nih.gov/pubmed/24988413

601. Xenaki, N.,Bacopoulou, F.,Kokkinos, A.,Nicolaides, N. C.,Chrousos, G. P.,Darviri C. Impact of a stress management program on weight loss, mental health and lifestyle in adults with obesity: a randomized controlled trial. J Mol Biochem. 2018. 7:78-84 https://www.ncbi.nlm.nih.gov/pubmed/30568922

602. Xu, B.,Houston, D. K.,Locher, J. L.,Ellison, K. J.,Gropper, S.,Buys, D. R.,Zizza, C. A. Higher Healthy Eating Index-2005 scores are associated with better physical performance. J Gerontol A Biol Sci Med Sci. 2012 67:93-9 https://www.ncbi.nlm.nih.gov/pubmed/22042723

603. Xu, F.,Letendre, J.,Bekke, J.,Beebe, N.,Mahler, L.,Lofgren, I. E.,Delmonico, M. J. Impact of a program of Tai Chi plus behaviorally based dietary weight loss on physical functioning and coronary heart disease risk factors: a community-based study in obese older women. J Nutr Gerontol Geriatr. 2015. 34:50-65 https://www.ncbi.nlm.nih.gov/pubmed/25803604 


$X \quad X$

Akaike, M.,Shimabukuro, M.,Katoh, S.,Sata, M. Association of lower limb muscle
Akaike, mass and energy expenditure with visceral fat mass in healthy men. Diabetol Metab Syndr. 2014. 6:27 https://www.ncbi.nlm.nih.gov/pubmed/24571923

605. Yamauti, A. K.,Ochiai, M. E.,Bifulco, P. S.,de Araujo, M. A.,Alonso, R. R.,Ribeiro, R. H.,Pereira-Barretto, A. C. Subjective global assessment of nutritional status in cardiac patients. Arq Bras Cardiol. 2006. 87:772-7 https://www.ncbi.nlm.nih.gov/pubmed/17262116

606. Yang, L. J.,Wu, G. H.,Yang, Y. L.,Wu, Y. H.,Zhang, L.,Wang, M. H.,Mo, L. Y.,Xue, G.,Wang, C. Z.,Weng, X. F. Nutrition, Physical Exercise, and the Prevalence of Sarcopenia in Elderly Residents in Nursing Homes in China. Med Sci Monit. 2019. 25:4390-4399 https://www.ncbi.nlm.nih.gov/pubmed/31189870

607. Yates, S. M.,Dunnagan, T. A. Evaluating the effectiveness of a home-based fall risk reduction program for rural community-dwelling older adults. J Gerontol A Biol Sci Med Sci. 2001. 56:M226-30 https://www.ncbi.nlm.nih.gov/pubmed/11283195

608. Yeo, W. K.,Lessard, S. J.,Chen, Z. P.,Garnham, A. P.,Burke, L. M.,Rivas, D. A., Kemp, B. E.,Hawley, J. A. Fat adaptation followed by carbohydrate restoration increases AMPK activity in skeletal muscle from trained humans. J Appl Physiol (1985). 2008. 105:1519-26 https://www.ncbi.nlm.nih.gov/pubmed/18801964

609. Yokoyama, Y.,Nishi, M.,Murayama, H.,Amano, H.,Taniguchi, Y.,Nofuji, Y.,Narita, M.,Matsuo, E.,Seino, S.,Kawano, Y.,Shinkai, S. Dietary Variety and Decline in Lean Mass and Physical Performance in Community-Dwelling Older Japanese: A 4-year Follow-Up Study. J Nutr Health Aging. 2017. 21:11-16 https://www.ncbi.nlm.nih.gov/pubmed/27999844

610. Yong, M. H.,Shin, J. I.,Yang, D. J.,Yang, Y. A. Comparison of Physical Fitness Status between Middle-aged and Elderly Male Laborers According to Lifestyle Behaviors. J Phys Ther Sci. 2014. 26:1965-9 https://www.ncbi.nlm.nih.gov/pubmed/25540509

611. Yoo, J. I.,Ha, Y. C.,Kwon, H. B.,Lee, Y. K.,Koo, K. H.,Yoo, M. J. High Prevalence of Sarcopenia in Korean Patients after Hip Fracture: a Case-Control Study. J Korean Med Sci. 2016. 31:1479-84 https://www.ncbi.nlm.nih.gov/pubmed/27510394

612. Yoshii, N.,Sato, K.,Ogasawara, R.,Kurihara, T.,Hamaoka, T.,Fujita, S. Relationship between Dietary Protein or Essential Amino Acid Intake and Training-Induced Muscle Hypertrophy among Older Individuals. J Nutr Sci Vitaminol (Tokyo). 2017. 63:379-388 https://www.ncbi.nlm.nih.gov/pubmed/29332899

613. Yoshimura, E.,Kumahara, H.,Tobina, T.,Matsuda, T.,Watabe, K.,Matono, S.,Ayabe M.,Kiyonaga, A.,Anzai, K.,Higaki, Y.,et al., Aerobic exercise attenuates the loss of skeletal muscle during energy restriction in adults with visceral adiposity. Obesity facts. 2014. 7:26-35 https://www.cochranelibrary.com/central/doi/10.1002/central/CN-00986322/full

614. Z. Movassagh E,Kontulainen, S.,Baxter-Jones, A. D. G.,Whiting, S.,Szafron, M., Papadimitropoulos, M.,Vatanparast, H. Are milk and alternatives and fruit and vegetable intakes during adolescence associated with cortical and trabecular bone structure, density, and strength in adulthood?. Osteoporos Int. 2017. 28:609-619 https://www.ncbi.nlm.nih.gov/pubmed/27699440

$\mathrm{X}$
$x$

$\mathrm{X}$



effects of a ketogenic diet on exercise metabolism and physical performance in offroad cyclists. Nutrients. 2014. 6:2493-508

https://www.ncbi.nlm.nih.gov/pubmed/24979615

616. Zbeida, M.,Goldsmith, R.,Shimony, T.,Vardi, H.,Naggan, L.,Shahar, D. R. Mediterranean diet and functional indicators among older adults in non-

Mediterranean and Mediterranean countries. J Nutr Health Aging. 2014. 18:411-8 https://www.ncbi.nlm.nih.gov/pubmed/24676323

617. Zhu, J.,Xiang, Y. B.,Cai, H.,Li, H.,Gao, Y. T.,Zheng, W.,Shu, X. O. A Prospective Investigation of Dietary Intake and Functional Impairments Among the Elderly. Am J Epidemiol. 2018. 187:2372-2386 https://www.ncbi.nlm.nih.gov/pubmed/30060001

618. Zhu, K.,Kerr, D. A.,Meng, X.,Devine, A.,Solah, V.,Binns, C. W.,Prince, R. L. TwoYear Whey Protein Supplementation Did Not Enhance Muscle Mass and Physical Function in Well-Nourished Healthy Older Postmenopausal Women. J Nutr. 2015. 145:2520-6 https://www.ncbi.nlm.nih.gov/pubmed/26400966

619. Zhu, N.,Jacobs, D. R.,Meyer, K. A.,He, K.,Launer, L.,Reis, J. P.,Yaffe, K.,Sidney, S.,Whitmer, R. A.,Steffen, L. M. Cognitive function in a middle aged cohort is related to higher quality dietary pattern 5 and 25 years earlier: the CARDIA study. $J$ Nutr Health Aging. 2015. 19:33-8 https://www.ncbi.nlm.nih.gov/pubmed/25560814

620. Zonta, M. L., Oyhenart, E. E.,Navone, G. T. Nutritional vulnerability in Mbya-Guarani adolescents and adults from Misiones, Argentina. Am J Hum Biol. 2011. 23:592600 https://www.ncbi.nlm.nih.gov/pubmed/21681847

$\mathrm{X}$

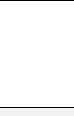

$x$

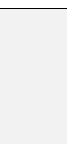

$\mathrm{x}$

$x$

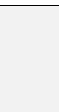

$\mathrm{X}$ $\mathrm{X}$

$\mathrm{X}$

X

